\title{
Methods for Detecting Unsolvable Planning Instances using Variable Projection
}

\section{Simon Ståhlberg}

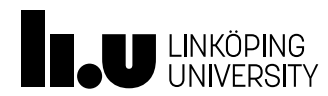

Linköping University

Department of Computer and Information Science Division for Software and Systems

SE-581 83 Linköping, Sweden 
(C) Simon Ståhlberg, 2017

ISBN 978-91-7685-498-3

ISSN 0345-7524

URL http://urn.kb.se/resolve?urn=urn:nbn:se:liu:diva-139802/

Published articles have been reprinted with permission from the respective copyright holder.

Typeset using LTEX

Printed by LiU-Tryck, Linköping 2017 


\section{Abstract}

In this thesis we study automated planning, a branch of artificial intelligence, which deals with the construction of plans. A plan is typically an action sequence that achieves some specific goal. In particular, we study unsolvable planning instances, i.e. when there is no plan. Historically, this topic has been neglected by the planning community, and up to recently the International Planning Competition only evaluated planners on solvable planning instances. For many applications we can know, for instance by design, that there is a solution, but this cannot be a general assumption. One example is penetration testing in computer security, where a system is considered safe if there is no plan for intrusion. Other examples are resource bounded planning instances that have insufficient resources to achieve the goal.

The main theme of this thesis is to use variable projection to prove unsolvability of planning instances. We implement and evaluate two planners: the first checks variable projections with the goal of finding an unsolvable projection, and the second builds a pattern collection to provide dead-end detection. In addition to comparing these planners to existing planners, we also utilise a large computer cluster to statistically assess whether they can be optimised further. On the benchmarks of planning instances that we used, it turns out that further improvement is likely to come from supplementary techniques rather than further optimisation. We pursue this and enhanced variable projections with mutexes, which yields a very competitive planner. We also inspect whether unsolvable variable projections tend to be composed of variables that play different roles, i.e. they are not 'similar'. We devise a variable similarity measure to rate how similar two variables are, and statistically analyse this. The measure can differentiate between unsolvable and solvable planning instances quite well, and is integrated into our planners. We also define a binary version of the measure, namely, that two variables are isomorphic if they behave in exactly the same way in some optimal solution (extremely similar). With the help of isomorphic variables we identify a computationally tractable class of planning instances that meet certain restrictions. There are several special cases of this class that are of practical interest, and this result encompass them.

This work has been supported by the Theoretical Computer Science Laboratory, Department of Computer and Information Science, Linköping University, and in part by CUGS (the National Graduate School in Computer Science, Sweden). 



\section{Populärvetenskaplig sammanfattning}

I den här avhandlingen studerar vi automatisk planering, vilket är ett nyckelområde inom artificiell intelligens. Planering är att tänka innan man handlar för att uppnå ett specificerat mål. Motsatsen till detta är att enbart handla reaktivt på vad som sker ögonblickligen. Planering använder sig av kunskap om världen för att konstruera en plan för vad som behöver göras, och i vilken ordning. Normalt tar vi inte hänsyn till allt i den riktiga världen och gör en avgränsning. Denna avgränsning kallas oftast för domän, och tre exempel på mycket olika domäner är logistik, schemaläggning, och pussel som sudoku. För sudoku är det till exempel onödigt att överväga dagens temperatur eftersom det inte finns någon koppling mellan de två, så inom denna värld har vi ingen kunskap om temperaturer. Med andra ord, inom planering studerar vi hur man uppnår mål inom olika avgränsningar av verkligheten. Det är viktigt att poängtera att det inte är ett fåtal domäner som vi studerar, trots allt, en artificiell intelligens måste, precis som en människa, kunna hantera många olika domäner och domäner som inte påträffats tidigare.

Den mest generella formen av automatisk planering är oavgörbar, vilket betyder att det inte finns en planerare som alltid kan konstruera (korrekta) planer. Det finns helt enkelt för många möjligheter vilket gör världen ohanterbar. Därför är det vanligt att anta, bland annat, följande förenklingar om världen: att vi känner till det nuvarande världstillståndet till fullo; att handlingar har exakt ett förutsägbart utfall; att handlingar utförs omedelbart; att inga handlingar kan utföras samtidigt; och att endast vi kan påverka världstillståndet. Med världstillståndet menar vi det kollektiva tillstånd som alla ting i (den avgränsade) världen har. Det kan tyckas att dessa begränsningar är väldigt restriktiva, men det finns 
många exempel som uppfyller dessa krav, exempelvis, pusslen Rubiks kub och sudoku. Det går också att använda denna typ av planering för planeringsproblem som inte uppfyller kraven genom att se planerna som riktlinjer; om man vill besöka någon plats och behöver bestämma en rutt är det i de flesta fall ointressant att överväga risken att fordonet går sönder under färden, eller hur alla andra trafikanter kan tänkas bete sig - det är något som hanteras då. Trots alla restriktioner är denna typ av planering inte enkel, om man inte angriper planering på ett klokt sätt så går det bara att konstruera planer för de allra enklaste planeringsproblemen inom rimlig tid.

$\mathrm{Nu}$ när vi har beskrivit vad automatisk planering är, och vilken typ av planering vi betraktar, är det dags att förklara vad avhandlingen behandlar. En vanlig metodik för att utvärdera hur bra en ny planerare fungerar är att låta den konstruera planer för en stor mängd planeringsproblem. Vanligtvis är dessa planeringsproblem tagna från planeringstävlingen International Planning Competition, och de ska exemplifiera planeringsproblem från verkligheten. I denna avhandling identifierade vi en anomali: att alla dessa planeringsproblem var lösbara! Det vill säga, för lösbara planeringsproblem så går det alltid att uppnå målet, och när man gav olösbara planeringsproblem till många moderna planerare så presterade de dåligt. Större delen av avhandlingen studerar olösbara planeringsproblem och beskriver flertalet planerare som presterar mycket bättre på sådana planeringsproblem. Stommen i våra planerare är variabelprojicering, vilket är en metod att förenkla planeringsproblem på ett sådant sätt att olösbarhet behålls. Det vill säga, om variabelprojektionen av ett planeringsproblem är olösbar så är planeringsproblemet också olösbart. Denna egenskap utnyttjas i olika avseenden av alla planerare i avhandlingen. Ett tillvägagångsätt är att systematiskt variabelprojicera på alla möjliga vis för att undersöka om det finns en olösbar variabelprojektion. Vår första planerare gör exakt detta, och undersöker variabelprojektionerna som är enklast först. Vår andra planerare bygger sedan vidare på denna idé genom att också utvinna kunskap ur de variabelprojektionerna som har undersökts. Båda planerarna presterar betydligt bättre än planerare som är optimerade för lösbara planeringsproblem.

Utöver planerarna har vi också gjort en detaljerad statistisk analys av variabelprojicering, då det finns ett ofantligt antal sätt att förenkla planeringsproblem. Denna analys togs fram med hjälp av ett stort beräkningskluster, och med denna data kunde vi bekräfta att planerarna använde sig utav variabelprojicering på ett nära optimalt vis. Detta 
innebär att vidare förbättringar måste utnyttja ytterligare metoder, vilket är något vi också undersöker. Slutligen identifierar vi även en grupp av planeringsproblem som går att lösa snabbt. Dessa planeringsproblem har en specifik struktur, vilket är av intresse i de fall en planerare stöter på ett planeringsproblem med denna struktur eftersom strukturen kan utnyttjas för att konstruera en plan snabbare, om det finns en. Strukturen av planeringsproblemen gav oss också insikt i varför vissa variabelprojektioner är olösbara och andra inte. 



\section{Acknowledgements}

It has been quite an adventure. My supervisors, Peter Jonsson and Christer Bäckström, also supervised my master's thesis and during it Peter asked me if I was interested in a $\mathrm{PhD}$ programme. I had not considered it at the time and it piqued my interest. Thank you, Peter, for this opportunity that you gave me. When I embarked on this adventure, I had a different understanding of the scope of it and many of the intricacies of it took me by surprise. One aspect that I found especially challenging was academic writing, and I must thank my advisors Peter Jonsson and Christer Bäckström for helping me with this by pointing out many oddities in my writings.

I must also thank the other members of the laboratory of theoretical computer science for providing and contributing to this unique experience: Meysam Aghighi, who I also wrote a paper with; Victor Lagerkvist; Hannes Uppman; and Biman Roy. It has been a pleasure.

Lastly, I want to thank my friends and family for their support and encouragement during this time.

Simon Ståhlberg Linköping, August, 2017 



\section{List of Publications}

This thesis consists of results built up over the course of several years, and a large portion of it has been published previously. Specifically, this thesis contains results from the following publications:

- Christer Bäckström, Peter Jonsson, and Simon Ståhlberg. Fast detection of unsolvable planning instances using local consistency. In Proceedings of the 6th International Symposium on Combinatorial Search (SoCS '13), pages 29-37, 2013.

- Meysam Aghighi, Peter Jonsson, and Simon Ståhlberg. Tractable cost-optimal planning over restricted polytree causal graphs. In Proceedings of the 29th AAAI Conference on Artificial Intelligence (AAAI '15), pages 3225-3231, 2015.

- Meysam Aghighi, Christer Bäckström, Peter Jonsson, and Simon Ståhlberg. Refining complexity analyses in planning by exploiting the exponential time hypothesis. Annals of Mathematics and Artificial Intelligence (AMAI), pages 157-175, 2016.

- Simon Ståhlberg. Tailoring pattern databases for unsolvable planning instances. In Proceedings of the 27th International Conference on Automated Planning and Scheduling (ICAPS '17), pages 274$282,2017$.

The author has also contributed to the following publication.

- Meysam Aghighi, Christer Bäckström, Peter Jonsson, and Simon Ståhlberg. Analysing approximability and heuristics in planning using the exponential-time hypothesis. In Proceedings of the 22nd European Conference on Artificial Intelligence (ECAI '16), pages $184-192,2016$. 



\section{Contents}

Abstract

Populärvetenskaplig sammanfattning iii

Acknowledgements vii

List of Publications $\quad$ ix

1 Introduction 1

1.1 Classical Planning ................ 3

1.2 Computational Complexity Theory . . . . . . . . 7

1.2.1 Asymptotic Complexity ............ 8

1.2.2 Complexity Classes ............... . 9

1.3 Contributions \& Thesis Outline . . . . . . . . . 10

$\begin{array}{lll}\text { I Preliminaries } & 15\end{array}$

2 Planning Formalism $\quad 17$

2.1 States and Reachability . . . . . . . . . . . 19

2.2 Variable Projection . . . . . . . . . . . . . . 20

2.3 Graphs.................... 21

2.4 Computational Problems . . . . . . . . . . . 23

3 Solvable vs. Unsolvable Instances 25

3.1 Exponential Time Hypothesis . . . . . . . . . . . 26

3.2 Solvable vs. Unsolvable Instances . . . . . . . . . . . . 27 
4 Planners \& Benchmarks $\quad 31$

4.1 Classical Planning Toolkit . . . . . . . . . . . . . . . . 31

4.1.1 Search Algorithms . . . . . . . . . . . . . 32

4.1.2 Performance Comparison . . . . . . . . . . 37

4.2 Benchmarks of Unsolvable Instances . . . . . . . . . . . 38

II Variable Projection 41

5 Consistency Checking 43

5.1 Definitions . . . . . . . . . . . . . . . . 43

5.2 Complexity Analysis . . . . . . . . . . . . . . 46

5.3 Statistical Analysis . . . . . . . . . . . . . . . . 48

5.3.1 Unsolvable Projections . . . . . . . . . . . . . . . 49

5.3 .2 Neighbour Projections . . . . . . . . . . . . . 50

5.3.3 Predecessor and Successor Projections . . . . . . 54

5.4 Experimental Analysis . . . . . . . . . . . . . . . 56

5.4 .1 Planners . . . . . . . . . . . . . . . 58

5.4 .2 Coverage . . . . . . . . . . . . . . . . 59

5.4 .3 Discussion . . . . . . . . . . . . 61

6 Pattern Databases $\quad 63$

6.1 Definitions . . . . . . . . . . . . . . . . . 64

6.2 Methods . . . . . . . . . . . . . . . 65

6.3 Statistical Analysis . . . . . . . . . . . . . . . . 66

6.3.1 Dead-end Detection . . . . . . . . . . 67

6.3.2 Unsolvable Projections . . . . . . . . . . . . . . 71

6.4 Mutual Exclusions . . . . . . . . . . . . . . . . . 72

6.5 Experimental Evaluation . . . . . . . . . . . . . 74

6.5.1 Planners . . . . . . . . . . . . . 75

6.5 .2 Coverage . . . . . . . . . . 76

6.5.3 Discussion . . . . . . . . . . . . 79

III Polytree Causal Graphs \& Variable Similarity 83

7 Tractable Classes $\quad 85$

7.1 Preliminaries . . . . . . . . . . . . . . 86

7.2 Bounded Diameter . . . . . . . . . . . . . 87

7.2 .1 Isomorphism . . . . . . . . . . . . . 88

7.2 .2 Defoliation of Polytrees . . . . . . . . . . 90 
7.2.3 The Algorithm . . . . . . . . . . . . . . . . 95

7.2 .4 Correctness . . . . . . . . . . . . . . 97

7.2 .5 Time Complexity . . . . . . . . . . . . . . . . 98

7.2 .6 Discussion . . . . . . . . . . . . . . . 100

7.3 Bounded Depth . . . . . . . . . . . . . . . . 102

7.3.1 Instance Splitting . . . . . . . . . . . . . 103

7.3 .2 Algorithm . . . . . . . . . . . . . . . . . 108

7.3 .3 Complexity . . . . . . . . . . . . . . . 112

7.3.4 Soundness, Completeness \& Optimality . . . . . 114

7.3 .5 Discussion . . . . . . . . . . . . . 116

8 Variable Similarity 119

8.1 Definitions . . . . . . . . . . . . . . . 120

8.2 Statistical Analysis . . . . . . . . . . . . . . . . . . . 122

8.3 Improving Consistency Checking . . . . . . . . . . . . . . . 122

8.4 Experimental Evaluation . . . . . . . . . . . . 126

8.5 Discussion . . . . . . . . . . . . . . . . . . 129

IV Concluding Remarks $\quad 131$

9 Concluding Remarks $\quad 133$

$\begin{array}{ll}\text { Bibliography } & 139\end{array}$ 



\section{Chapter One Introduction}

The contributions of this thesis are related to the area of automated planning, or simply planning, which is one of the main research areas of artificial intelligence (AI) and has been so since the beginning of the field. Before we go into detail of what planning is and what our contributions are, we give a concise overview of the role that planning plays in the broader context of AI. As the name AI suggests, a long-term goal of the field is for some artifact to exhibit intelligent thought process or behaviour. Currently, the artifact is computers, but that might change with future inventions. The latter, however, is philosophical by nature and there is a myriad of definitions of intelligence, where every definition has its own ambition. Four simple definitions are that the artifact should: think like humans; behave like humans; think rationally; or behave rationally. For example, robots were recently introduced to the healthcare system to care for elderly, such as helping them stand, walk, or even alleviating loneliness [RMKB13]. In this case we want robots to behave like humans, especially regarding alleviating loneliness since a pure rational robot is unlikely to be suitable for human interaction (see Dautenhahn [Dau07] for a description of social rules for robots). Additionally, Watson from IBM, originally built for Jeopardy! $\left[\mathrm{FBCc}^{+} 10\right.$, Fer12], was recently specialised to diagnose cancer patients $\left[\mathrm{FLB}^{+} 13\right]$. In this instance we want the system to think rationally and suggest correct treatments.

Note that we have not formally defined what intelligence is, just that an AI either imitates us or is rational. Both, at its core, depend on our subjective opinion (i.e. how do we define rational behaviour?). There is no formal definition of what intelligence is, and there is no list of qualifications for when we have created something intelligent. One attempt to test when we have achieved our goal is the Turing test by Alan Turing in 1950 [Tur50]. The AI passes the test if a human 
interrogator, after asking questions, cannot decide whether the responses are from an $\mathrm{AI}$ or a human. The interrogator is in a different room than the AI since having a phyiscal body is not a requirement for intelligence. The total Turing test also includes a physical body. Again, Turing used human-like behaviour as a measurement of intelligence. Many computer programs, that are clearly not intelligent, frequently passes variants of the Turing test. Hence, the test itself is widely criticised, but gave insights into what might be necessary to pass a thorough version. To pass a thorough version of the total Turing test, the AI might need to be able to do at least the following.

- Knowledge representation. The AI might require knowledge about the world to function, such as objects (doors and keys) and properties (doors can be locked and unlocked), but also less tangible things like concepts, categories and relations (some keys lock and unlock doors).

- Automated reasoning. If the AI does not have complete knowledge of the world, then she might need to use reasoning to derive further knowledge. One very active subarea of this field is automated theorem proving.

- Automated planning. The AI might need to perform actions in the world to achieve its goal. Planning deals with the construction of strategies, or action sequences, that achieves the goal. It is important to note that the plan is contructed ahead of time and the AI has to consider expected and unexpected results. This thesis contributes to this area.

- Machine learning. If the AI does not have a complete understanding of the world, then it might sometimes fail. In this case, it is important for the AI to have the ability to learn.

- Natural language processing. In order to communicate with us, the AI needs to understand both written and spoken languages. Strictly speaking, natural language processing is not a necessity for intelligence (this is also true for the capabilities beneath).

- Perception. The AI might need to take input from sensors, such as cameras (computer vision), microphones, and so on, to extract knowledge about its surroundings. 
- Robotics. The AI might need to navigate and manipulate its surroundings. Two subareas of this field are motion planning and path planning, which should not be confused with automated planning (a more general case).

A long-term goal is to develop a strong $A I$, which is a term reserved for AIs that are able to do nearly all in the list. In contrast, a weak AI focuses on a narrow task, and there are many such examples in the real world. One example is the planning system part of the Deep Space One spacecraft from NASA $\left[\mathrm{JMM}^{+} 00\right]$, which monitored the operation of the spacecraft and that its plans were executed - if not, then it diagnosed and repaired the plan. The planning system was necessary because direct control is unreasonable due to the communication delay. Another example is the emerging technology of rescue robots, which are robots used in disasters to search for and rescue people. Two examples of disasters are mining accidents and earthquakes, and in such situations it might be dangerous for rescue personnel to undertake certain tasks, and it is appropriate to use robots. Furthermore, some robots are more apt at surveying narrow spaces, and if they can navigate autonomously then personnel can be used more efficiently. The international RoboCup $\left[\mathrm{KAK}^{+} 97\right]$ competition has a league for rescue robots. Other examples are AIs for games such as chess and go, and AIs for these games have defeated world champions [Pan97, $\mathrm{SHM}^{+} 16$, Orm16].

The field that this thesis expands on is automated planning, which is unmistakably important to build many functional AIs. This introductionary chapter is divided into three sections. Section 1.1 presents the planning problem that we study in high-level terms, and Section 1.2 explains what computational complexity is. Finally, Section 1.3 outlines and gives a summary of the results in this thesis.

\subsection{Classical Planning}

Within most areas of artificial intelligence, the notion of an intelligent agent is fundamental. An agent lives in a world, which she can perceive and act in. The world consists of entities, such as doors, keys, and agents (we use the term variable instead of entity later on). At any particular point in time, every entity has a state and the state of the world is the collective state of its entities. The agent can perform actions in the world, such as opening a door. Of course, the aspiration is for the world model to be as flexible as the real world, but we restrict the 
world by imposing some assumptions on it. For instance, many different worlds can be defined by assuming some of the following properties.

- Deterministic vs. stochastic. The outcome of every action is predictable in a deterministic world.

- Discrete vs. continuous. The world is discrete if the set of possible states for every entity is discrete.

- Finite vs. infinite. The world is finite if the set of possible states for every entity is finite.

- Fully observable vs. partially observable. The world is fully observable if every agent can always perceive the state of every entity.

- Static vs. dynamic. The world is static if only the actions of agents can change the state of the world.

Clearly, there is no lack of assumptions to make about the real world. With respect to these properties, the classical world is the simplest possible one: the number of states of the world is finite and discrete, every agent can fully observe it, and only the deterministic actions of the agents can change its state. It might seem like this problem is too simple, are such worlds even encountered in the real world? Indeed, puzzles such as crosswords and Rubik's Cube, as well as board games like chess and go, immediately meet these assumptions. Many tasks such as plotting a route to drive can be viewed as a classical planning problem; at a high-level we usually do not contemplate the risk of a vehicle breakdown or other road users, and maps are easily translated to some graph structure.

The planning problem deals with the construction of a plan, which achieves a goal in a world for one or more agents. In addition to the restrictions discussed earlier, we also consider the following.

- If the initial state of the world is known.

- If actions have durations.

- If actions can be performed concurrently.

- If the goal is to reach some specific state, or to maximise some reward function.

- If there are several agents, and if they are co-operative. 


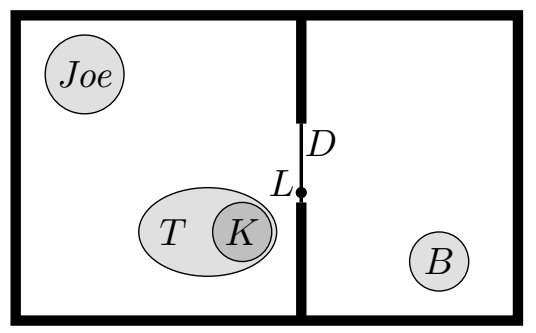

Figure 1.1: An illustration of the initial state of Example 1.1.

These properties are relevant to the agent(s) in the world and not the world itself. The classical planning problem is the simplest problem. In other words, the single agent lives in the classical world and knows the initial state. Her actions have no duration and cannot be performed concurrently. The goal is for the world to be in a specific state.

Example 1.1. Consider a world of two rooms $R_{1}$ and $R_{2}$, which are connected by a locked door $D$. There is no window between the rooms. Joe lives in $R_{1}$. He is a gorilla with a passion for consuming bananas. Sadly, he has not been able to enjoy his passion lately, but he has a plan. Joe noticed, by using his X-ray vision, that there is a bunch of bananas $B$ in $R_{2}$. Specifically, musa acuminata - his favourite species of bananas. However, the door to $R_{2}$ is locked, and the key $K$ for the door is 'hidden' inside a toy $T$ in his room. Arguably, it is not possible to hide anything from Joe because of his awesome power. The toy is a Rubik's Cube and he believes that the cube opens up when solved. Joe's plan is simple: take $T$; solve $T$; grab $K$; unlock $D$ with $K$; open $D$; enter $R_{2}$; pick up $B$; and finally, munch on $B$.

Example 1.1 (together with Figure 1.1) is meant to illustrate a classical planning problem, and in Chapter 2 we show how to formally model the example. The keen reader might have noticed that Joe is not your typical gorilla: he is blessed with X-ray vision. This is a consequence of Joe living in a classical world, which is fully observable. Joe's plan is a high-level plan and there are many gaps that needs to be filled in. For instance, how to solve the Rubik's Cube is left out, and how to execute the plan in terms of motor control (robotics) is omitted. Such intricate details of a plan are often delegated to other systems to be filled in.

If Joe's plan does not succeed for some reason, then he has to revisit it. An execution monitoring agent checks if everything is going 
according to the plan. There are several types of execution monitoring, for instance, action monitoring, which verify that the next action is viable and if not then it might be possible to repair the plan (e.g. replan some part of it). However, both execution monitoring and plan repair are outside the scope of this thesis. In some sense, the properties of classical planning allow us to construct high-level plans. It is important to note that the plan in Example 1.1 was constructed in advance. The initial state of the static world was fully known and the actions were deterministic. These three properties allow us to devise a plan (if there is one) ahead of time that is guaranteed to achieve the goal (with respect to the model).

It might be tempting to think that classical planning is easy after imposing so many restrictions on the world. Figure 1.1 depicts the initial state of Example 1.1, but how many states are there? To answer that question, we need to know the states of every entity.

Example 1.2. One way to represent the states of every entity of Example 1.1 is the following. The possible states of:

- Joe are $R_{1}$ and $R_{2}$ (the rooms Joe can be in),

- $T$ are Solved and Unsolved,

- $K$ are $T$ and Joe (either the key is inside the cube, or Joe has it),

- $L$ are Locked and Unlocked,

- $D$ are Closed and Open, and

- $B$ are Not-eaten and Eaten.

We say that the set of these values is the domain of the entity, e.g. the domain of Joe is $\left\{R_{1}, R_{2}\right\}$. The initial state is represented as the set $\left\{\left(\right.\right.$ Joe,$\left.R_{1}\right),(T$, Unsolved $),(K, T),(L$, Locked $),(D$, Closed $),(B$, Noteaten $)\}$. The first element of every pair is the entity, whereas the second element is the state of the entity. One state that Joe wants to be in is the goal state $\left\{\left(\right.\right.$ Joe,$\left.R_{2}\right),(T$, Solved $),(K$, Joe $),(L$, Unlocked $),(D$, Open $)$ $(B$, Eaten $)\}$. The number of different states is $2^{6}=64$. However, some states might not be reachable from the initial state, i.e. there are no plans from the initial state to them. For example, the state $\left\{\left(\right.\right.$ Joe,$\left.R_{2}\right),(T$, Unsolved $),(K, T),(L$, Locked $),(D$, Closed $)(B$, Eaten $\left.)\right\}$ is unreachable since there is no way for Joe to be in $R_{2}$ without opening the door. 
The number of states of Example 1.2 is only 64, which might not sound like a lot. However, if we were to place an additional 260 bananas in $R_{2}$, then the number of states would be greater than the number of atoms in the observable universe. ${ }^{1}$ The reason for this absurd increase in the amount of states is that Joe can eat the bananas in very many different orders. Every different combination of Eaten and Not-Eaten is an unique state. This effect is typically described as a combinatorial explosion. Note that the same thing happens when we introduce irrelevant entities, for instance, suppose there are a lot of retractable pens on the floor and Joe can extend the tip of each pen by clicking on top of it. This also causes a combinatorial explosion of states. However, the pens are irrelevant for Joe's plan. The problem is still easy for humans since we are aware of the fact that there is no need to differentiate between the bananas, or that we can ignore the pens, but automated planners cannot differentiate. Of course, in the general case, we have to differentiate between every entity and such problems would also be extremely difficult for humans. Much research in this field has been devoted to how to deal with the combinatorial explosion of states. In other words, even classical planning is computationally hard, which is the theme of our next discussion.

\subsection{Computational Complexity Theory}

Planning problems can differ vastly regarding computational complexity. The objective of computational complexity is to classify problems according to their inherent difficulty, and compare the difficulties to one another. We assume that the reader is already familiar with computational complexity, and give a brief reminder. Consider the following computational problem.

\section{SAT}

InSTANCE: A boolean formula $F$ on conjunctive normal form, i.e. $F$ is a conjunction of clauses where each clause is a disjunction of literals.

Question: Does $F$ have a satisfying assignment?

We require, without loss of generality, that repeated clauses are not allowed and that no empty clauses appear. Note that the definition of SAT instances implies that there are no unused variables, i.e. every

\footnotetext{
${ }^{1}$ The number of atoms in the observable universe is estimated to be $10^{80}$.
} 
variable appears in at least one clause. The problem $k$-SAT, $k \geq 1$, is the SAT problem restricted to clauses containing at most $k$ literals.

Example 1.3. The boolean formula $(x \vee y) \wedge(\neg y \vee \neg z)$ has the satisfying assignment $x=T, y=T, z=F$. The assignment of $y$ does not matter. The formula is on conjunctive normal form.

The answer to the SAT problem for the boolean formula in Example 1.3 is 'yes' since we have a satisfying assignment. An obvious algorithm to find such an assignment is to systematically enumerate every assigment, and check if any of them satisfies the boolean formula. Our main concerns are how efficient the algorithm is, and if there are more efficient algorithms. We denote this particular algorithm as $\mathcal{A}$ for the remainder of this section.

\subsubsection{Asymptotic Complexity}

Algorithm $\mathcal{A}$ answers the question of the SAT problem for a boolean formula $F$. Let $n$ be the number of variables in $F$, and $m$ be the length of $F$. Then the number of possible assignments for $F$ is $2^{n}$. Given an assignment, we can compute the next assignment in $n$ steps. Furthermore, we can check whether an assignment satisfies $F$ in roughly $m$ steps. (The details of how we do this are left as an exercise.) Consequently, the number of steps of $\mathcal{A}$ is, in the worst case, roughly $2^{n} \cdot n \cdot m$. A step can be thought of as a machine instruction for a computer, or a constant number of them, where the instruction takes constant time to execute. In this case, the number of steps measure how much time the algorithm require. Similarly, we can measure space, i.e. the memory usage of the algorithm.

The analysis of algorithm $\mathcal{A}$ exemplify the intuition behind asymptotic complexity: we ignore constant factors and terms and study how the algorithm behave when the input size approach infinity. The ignored factors and terms are dominated by the remaining parts when the input size is sufficiently large. Formally, let $f$ and $g$ be two functions defined on some subset of the real numbers. Then we write $f(x)=\mathcal{O}(g(x))$ if and only if there is a positive constant $c$ and a real number $x_{0}$ such that $f(x) \leq c \cdot g(x)$ for all $x \geq x_{0}$. Intuitively, the function $f$ does not grow faster than $g$ for sufficiently large $x$. For example, the asymptotic complexity of $\mathcal{A}$ is $2^{n} \cdot n \cdot m \leq 2^{m} \cdot m^{2}=\mathcal{O}\left(2^{m} \cdot m^{2}\right)(n<m$ since every variable occurs at least once in $F$ ). 


\subsubsection{Complexity Classes}

A complexity class is a set of problems of related complexity. We are especially interested in the following classes.

- P: The set of problems that can be solved by an algorithm running in time $\mathcal{O}\left(n^{c}\right)$, where $c$ is a constant. When we say that an algorithm is polynomial, then the problem it solves is in $\mathbf{P}$. If a problem is in this class, then we consider it to be tractable (computationally feasible), and otherwise to be intractable. (If this is a fair dichotomy is debatable.)

- NP: The set of problems that can be solved by a non-deterministic algorithm running in time $\mathcal{O}\left(n^{c}\right)$, where $c$ is a constant. A non-deterministic algorithm can be thought of as an algorithm that, when confronted with a several options, is always able to guess the correct option. For instance, algorithm $\mathcal{A}$ has to check every assignment since we do not know which one is a satisfying assignment, and if there is one. A non-deterministic version of $\mathcal{A}$ consists of two phases: a non-deterministic phase and then a deterministic phase. The first phase guesses an answer and the second phase verifies it. In this case, we would guess some assignment in the first phase, and then deterministically check if it satisfies the formula in the second phase. Assuming that every guess was correct, $\mathcal{A}$ is in NP since both phases runs in polynomial time. It is known that $\mathbf{P} \subseteq \mathbf{N P}$, but whether $\mathbf{P} \neq \mathbf{N P}$ is a major open question. However, due to the amount of research that has been devoted to answering just that question throughout many decades, it is common to assume that $\mathbf{P} \neq \mathbf{N P}$.

- PSPACE: The set of problems that can be solved by an algorithm running in space $\mathcal{O}\left(n^{c}\right)$, where $c$ is a constant. It is known that $\mathbf{N P} \subseteq \mathbf{P S P A C E}$, however if $\mathbf{N P} \neq \mathbf{P S P A C E}$ and $\mathbf{P} \neq$ PSPACE are also open questions.

As the reader might have noticed, there is a straightforward hierarchy of the complexity classes, but if a problem is, for instance, in PSPACE then we do not know how hard it is (since $\mathbf{P} \subseteq$ PSPACE). Hence, hardness with respect to a complexity class means, informally, that the problem is at least as hard as the hardest problems in the class. For example, the SAT problem is in NP and is also NP-hard [GJ79, problem LO1]. Furthermore, if a problem is both in NP and is NP-hard 
then we say that it is NP-complete (we use analogous definitions for other classes). Hardness can be formalised by using polynomial-time reductions, which translates, or reduces, one problem to another. The reduction must be done in polynomial-time, and the answer for the former problem is derived from the answer for the latter problem. If the latter problem can be answered quickly, then so can the former since the reduction is done in polynomial-time. Hence, the latter problem is at least as hard as the former. If a problem is NP-hard then it is common to assume that there is no polynomial algorithm which solves it (i.e. by assuming that $\mathbf{P} \neq \mathbf{N P}$ ). We refer the reader to the textbook by Papadimitriou [Pap94] for more information.

\subsection{Contributions \& Thesis Outline}

There has been an impressive advancement in domain-independent planning over the past decades. New and efficient planners have entered the scene, for instance, FAST Forward [HN01], FAST DOWNWARD [Hel06a], LAMA [RW10], and planners based on compilation into SAT instances [RHN06]. Various ways to exploit the structure of planning instances have been proposed and integrated into existing planners. Perhaps most importantly, a rich flora of heuristics has appeared, making many previously infeasible instances solvable with reasonable resources. See, for instance, Helmert et al. [HD09] for a survey and comparison of the most popular heuristics. This development has largely been driven by the international planning competitions (IPC). However, in the long run one might question how healthy it is for a research area to focus so much effort on solving the particular problems choosen for the competitions. This is a very natural question from a methodological point of view, and although it has not been raised as often as one might expect, it is not a new one, cf. Helmert [Hel06b].

One problem with the planning competitions so far is that all planning instances have a solution, and the effect of this is that the planners and methods used are getting increasingly faster at finding the solutions that we already know exist. It is important to note that other similar competitions, such as the SAT competition (http://www.satcompetition.org) and the Constraint Solver Competition (http://cpai.ucc.ie), consider unsolvable instances together with solvable instances. It is no secret that few planners exhibit any similar speed improvement for instances that have no solution. For many applications we can know, e.g. by design, that there is a solu- 
tion, but this cannot be a general assumption. Obvious examples are planning for error recovery and planning for systems that were not designed to always have a solution (old industrial plants that have evolved over time is an archetypical example). Another example is support systems where a human operator takes the planning decisions and the planner is used to decide if there is a plan or not, or to attempt finding errors in the human-made plan [GKS12]. System verification has an overlap with planning [ELV07], and proving that a forbidden state is unreachable corresponds to proving that there is no plan. Similarly, planning is used for penetration testing in computer security [BGHH05, SBH13, FNRS10, HGHB05], which is an area of emerging commercial importance [SP12]. In penetration testing, a system is considered safe if there is no plan for intrusion. We finally note that oversubscription planning (i.e. where not all goals can be satisfied simultaneously and the objective is to maximise the number of satisfied goals) have been considered in the literature [Smi04].

Perhaps the most important contribution of our work is that we sparked interest in the anomaly explained above by implementing a planner tailored towards unsolvable instances and demonstrated that it outperformed state-of-the-art IPC planners on such instances. This exposed a weakness which needed to be addressed by the planning community, and as a result a number of responses have been produced. The situation has improved significantly, and IPC has introduced an unsolvability track to encourage further research. The following publications are a few examples of recent methods for detecting unsolvable instances. Hoffman et al. [HKT14] specialised the heuristic function Merge \& Shrink [HHH07] for detecting unsolvable instances. Steinmetz and Hoffmann [SH16] tailored the heuristic function $h^{C}$ for detecting dead-end states, and gave it the ability to learn from misclassifications to improve itself. Lipovetzky et al. [LMG16] investigated the relation between traps and dead-ends, and devised a preprocessing algorithm for computing them. Gnad et al. $\left[\mathrm{GSJ}^{+} 16\right]$ designed a method around partial delete relexation, or red-black planning, to detect unsolvable planning instances. Gnad et al. [GTSH17] exploited symmetries in the planning instance to prune states during the search on its state transition graph. This is far from a complete list, but it is clear it sparked an interest and that a lot of experimentation is taking place. It is worth noting that dead-end detection is not something new, but its relevance to unsolvable planning instances was not studied in-depth. Some contenders from the first unsolvability IPC are Aidos [ $\left.\mathrm{SPS}^{+} 16\right]$, 
Django [GSH16], SymPA [Tor16], M+S and SimDominance [THK16]; and they make use of the results in the publications above and more. We compare our planners to some of these contenders, and we outline and describe our results next.

\section{Part I: Introduction}

In this part, we define the planning formalism that we use throughout the thesis (Chapter 2). We also motivate our work further by showing that there is no significant theoretical difference, with respect to complexity, between solvable and unsolvable instances (Chapter 3). Finally, we give a brief explanation of the inner workings of our planner (Section 4.1), and discuss the domains of unsolvable instances that we use (Section 4.2).

\section{Part II: Variable Projection}

This part describes two planners for unsolvable problems, both of them are based on variable projection, which is a technique to simplify instances. From now on, we use the more mathematical term variable instead of entity, they are essentially synonyms. Variable projection lets us project away variables from the instance, for example, if we were to project away the lock in Example 1.1 then we can open the door without the key. The key is still in the projected world, but it has no use. A plan for the projected instance is: open the door; go to the other room; dine on the banana. Note that this plan is an incomplete and inapplicable plan for the original instance, and to complete it we can add these actions: take the puzzle; solve the puzzle; grab the key; and unlock the door. In other words, variable projection lets us find partial plans that are parts of a plan for the instance. The process of completing a partial plan is also known as plan refinement; this is also how variable projection was used in the beginning [Kno94]. There is no guarantee that partial plans can be refined into a plan for the instance, however, if there is no partial plan at all for a projected instance then there is no plan for the original instance. This is a very important property: we can prove that an instance is unsolvable by proving that a simpler instance, constructed by variable projection, is unsolvable. This fact is the cornerstone which our planners are based on.

The first planner, consistency checking, systematically enumerates every subset of variables and projects the instance onto it, and then checks whether the projection is unsolvable or not. If the projection is unsolvable, then the original instance must be unsolvable. Projections 
onto smaller subsets are faster to solve, so the method starts with projecting onto every subset of size 1 , then every subset of size 2 , and so on, up to size $k$. Consistency checking for planning is often efficient, but at the cost of being incomplete, that is, it is infeasible to detect all unsolvable instances. Consistency checking also runs in polynomial time for any fixed value of a parameter $k$, which can be used to trade off between efficiency and completeness. Consistency checking for planning is efficient if unsolvability can be proved with a reasonably small variable subset, otherwise it quickly becomes too time-consuming because of the sheer number of possible projections.

The second planner is an adaptation of an existing heuristic function for solvable instances: pattern databases (PDB). More precisely, we adapt pattern collections. A pattern is the same as a projected instance, and we have a collection of different projected instances. Historically, PDBs have used variable projection for providing heuristic guidance and, naturally, such PDBs perform badly when given an unsolvable instance. We refocus PDBs to identify dead-end states - states from which no goal state is reachable. Note that if the instance is unsolvable, then the initial state is a dead-end state. Hence, dead-end detection is a viable approach to identify unsolvable instances.

We evaluate both planners on 183 unsolvable planning instances over 8 domains, matching our methods against some of the most commonly used planners. A typical statistical analysis of some method study instance coverage and run-time, but we also carry out a much more in-depth statistical analysis by taking advantage of a large computer cluster. One issue with the typical analysis is that the limitation of the methods is not clear. For example, if we have a PDB that performs well on an instance, then we do not know if there is another PDB which would have performed considerably better. With the help of the computer cluster, we establish a distribution for every instance of its projections, of various properties. The distributions help us answer questions such as 'how optimal is our method with respect to its potential?'. Note that we are discussing the potential, or 'upper bound', of the method as a whole. In other words, to improve the potential of the method, we have to complement it with additional techniques.

\section{Part III: Polytree Causal Graphs \& Variable Similarity}

We take a detour from unsolvable instances and look at a specific class of instances, which gave us insights in which variable projections are of particular interest when trying to detect unsolvability. The class 
consists of instances whose causal graph is a polytree. The causal graph of an instance is a directed graph of its variables, where an edge from one variable to another means that the former can influence the latter through an action. We also restrict the domain size of the variables (entities) by a constant, and either the depth or the diameter of the causal graph. The depth is a graph is the length of the longest simple

directed path, and the diameter is the longest simple undirected path. Similar classes has been studied previously, and some of them are special cases of these classes.

The algorithm for one of the classes works by recursively breaking down the instance into several smaller instances, which are then solved independently. A solution for the instance is then derived from the solutions for the smaller instances. The foundation of the algorithm for the other class is the notion of variable ismorphism. Essentially, two variables are isomorphic if one of them can be removed without affecting solvability because the two 'behave' the same anyway. Going back to Example 1.1, we mentioned that if we add 260 bananas then we get a combinatorial explosion, and we also mentioned that there was no need to differentiate between them. One way to motivate this is that the bananas are isomorphic. That is, we can remove all but one banana without affecting solvability. We show, by exploiting this feature, that the class defined in the previous paragraph is tractable, albeit the constants in the asymptotic complexity are large.

We follow up on this idea by defining a variable similarity measure, which scores the similarity of two variables between 0 and 1 . The similarity score of two isomorphic variables is always 1 , however, it is possible for two non-isomorphic variables to also get the score 1 . This is because it is infeasible to decide whether two variables are isomorphic in the general case, so we need an approximation. We then show empirically that the smallest unsolvable projections very often consist of variables that pairwise score very low on the variable similarity measure. This is important since we now have a tool that can help us decide whether to consider a variable projection. We use this measure to improve the planners in Part II. The tool is statistically analysed and the improvement is empirically evaluated.

\section{Part IV: Conclusions}

We conclude this thesis with a summary and discuss future research directions. The main topic is how to improve variable projection and thus the methods. We also mention some open research questions. 


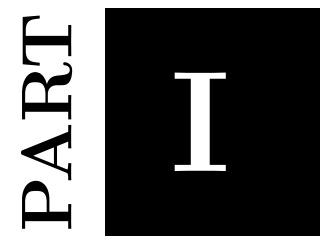

\section{Preliminaries}





\section{Chapter Two Planning Formalism}

In this chapter we present the planning formalism that is used in this thesis and show some important properties, discuss how planners are implemented in practice, and present the unsolvable instances that we evaluate our results on.

We use the extended simplified action structures $\left(\mathrm{SAS}^{+}\right)$[BN95] formalism, which uses multi-valued variables. This is the main difference between $\mathrm{SAS}^{+}$and STRIPS, which use propositional atoms (binary variables). $\mathrm{A} \mathrm{SAS}^{+}$instance is a tuple $\Pi=(V, A, I, G)$ where:

- $V=\left\{v_{1}, \ldots, v_{n}\right\}$ is the set of variables, and each variable is associated with a domain $D_{v}$. A partial state is a set $s \subseteq \bigcup_{v \in V}\{(v, d)$ : $\left.d \in D_{v}\right\}$ where every variable in $V$ occurs in at most one pair. A total state is a partial state where every variable in $V$ occurs in exactly one pair. We also view partial states as partial functions and use the corresponding notation, i.e. $s[v]=d$ means that $(v, d) \in s$, and $\operatorname{Domain}(s)=\{v:(v, d) \in s\}$ denotes the domain of $s$.

- $A$ is the set of actions. An action $a=\operatorname{pre}(a) \rightarrow \operatorname{eff}(a)$ consists of two partial states, a precondition pre $(a)$ and an effect $\operatorname{eff}(a)$. An action $a$ has a prevailcondition on a variable $v$ if $v \in$ $\operatorname{Domain}(\operatorname{pre}(a)) \backslash \operatorname{Domain}(\operatorname{eff}(a))$, i.e. there is a precondition but no effect on $v \cdot{ }^{1}$ Furthermore, we do not allow $\operatorname{pre}(a)[v]=\operatorname{eff}(a)[v]$ since the effect on $v$ is then superfluous.

- $I$ is the initial state, and it is a total state.

- $G$ is the goal, and it is a partial state.

\footnotetext{
${ }^{1}$ This is slightly non-standard, some formalisms define preconditions and prevailconditions of an action as distinct sets of $\operatorname{pre}(a)$. However, in the vast majority of this thesis we do not need to distinguish between the two.
} 
We write $V(\Pi), A(\Pi), I(\Pi)$ and $G(\Pi)$ for the set of variables, actions, the initial state and the goal of $\Pi$, respectively.

We say that a partial state $s_{1}$ matches another partial state $s_{2}$ if $s_{1} \subseteq s_{2}$. We define the composition of two partial states $s_{1}, s_{2}$ as $s_{1} \oplus s_{2}=s_{2} \cup\left\{\left(v, s_{1}[v]\right): v \in \operatorname{Domain}\left(s_{1}\right) \backslash \operatorname{Domain}\left(s_{2}\right)\right\}$. An action $a$ is applicable in a total state $s$ if $\operatorname{pre}(a) \subseteq s$, and the result of applying $a$ in $s$ is the total state $s \oplus \operatorname{eff}(a)$.

Example 2.1. Recall Example 1.2. The way we represented the entities, i.e. the variables, followed the $\mathrm{SAS}^{+}$formalism closely. Henceforth, we denote the following instance as $\Pi_{J o e}$. The set of variables is $V\left(\Pi_{J o e}\right)=$ $\{$ Joe $, T, K, L, D, B\}$, where:

- $D_{J o e}=\left\{R_{1}, R_{2}\right\}$ (the rooms Joe can be in),

- $D_{T}=\{$ Solved, Unsolved $\}$,

- $D_{K}=\{T, J o e\}$ (the key is either inside the cube, or Joe has it),

- $D_{L}=\{$ Locked, Unlocked $\}$,

- $D_{D}=\{$ Closed, Open $\}$, and

- $D_{B}=\{$ Not-eaten, Eaten $\}$.

The initial state is $I\left(\Pi_{\text {Joe }}\right)=\left\{\left(\right.\right.$ Joe, $\left.R_{1}\right),(T$, Unsolved $),(K, T)$, $(L$, Locked $),(D$, Closed $),(B$, Not-eaten $)\}$, and the goal is $G\left(\Pi_{J o e}\right)=$ $\{(B$, Eaten $)\}$ (Joe's life goal is to devour $B)$. The actions of $\Pi_{J o e}$ are the following.

- $a_{\text {Solve }}=\left\{\left(\right.\right.$ Joe,$\left.R_{1}\right),(T$, Unsolved $\left.)\right\} \rightarrow\{(T$, Solved $)\}$,

- $a_{\text {GrabKey }}=\left\{\left(\right.\right.$ Joe,$\left.R_{1}\right),(T$, Solved $\left.),(K, T)\right\} \rightarrow\{(K$, Joe $)\}$,

- $a_{\text {Unlock }}=\{(K$, Joe $),(L$, Locked $),(D$, Closed $)\} \rightarrow\{(L$, Unlocked $)\}$,

- $a_{\text {Lock }}=\{(K$, Joe $),(L$, Unlocked $),(D$, Closed $)\} \rightarrow\{(L$, Locked $)\}$,

- $a_{\text {Open }}=\{(L$, Unlocked $),(D$, Closed $)\} \rightarrow\{(D$, Open $)\}$,

- $a_{\text {Close }}=\{(D$, Open $)\} \rightarrow\{(D$, Closed $)\}$,

- $a_{R_{1} \rightarrow R_{2}}=\left\{(D\right.$, Open $),\left(\right.$ Joe,$\left.\left.R_{1}\right)\right\} \rightarrow\left\{\left(\right.\right.$ Joe,$\left.\left.R_{2}\right)\right\}$,

- $a_{R_{2} \rightarrow R_{1}}=\left\{(D\right.$, Open $),\left(\right.$ Joe,$\left.\left.R_{2}\right)\right\} \rightarrow\left\{\left(J_{o e}, R_{1}\right)\right\}$,

- $a_{\text {Ingest }}=\left\{\left(\right.\right.$ Joe,$\left.R_{2}\right),(B$, Not-eaten $\left.)\right\} \rightarrow\{(B$, Eaten $)\}$, 
Note that the cube cannot be moved around (it is always in $R_{1}$ ) and the same is true for $B$ (it is always in $R_{2}$ ). This is not a limitation of the $\mathrm{SAS}^{+}$formalism, we simply wanted a more brief example. For instance, if the domain of $B$ was $\left\{R_{1}, R_{2}\right.$, Joe,Eaten $\}$, then we could have had actions to let Joe pick it up and put it down.

\subsection{States and Reachability}

The plan that Joe conceived in Example 1.1 was a linear plan, which is the type of plans that we consider in this thesis. We are interested in whether a state is reachable from the initial state, and whether a goal state is reachable from another state. The following three definitions define these nuances.

Definition 2.2. Let $s_{0}$ and $s_{n}$ be two total states, and $\omega=\left\langle a_{1}, \ldots, a_{n}\right\rangle$ be sequence of actions. Then $\omega$ is a plan from $s_{0}$ to $s_{n}$ if and only if there exists a sequence of intermediate total states $\left\langle s_{1}, \ldots, s_{n-1}\right\rangle$ such that $a_{i}$ is applicable in $s_{i-1}$ and $s_{i}$ is the result of applying $a_{i}$ in $s_{i-1}$ for all $1 \leq i \leq n$. The plan $\omega$ is also a solution with respect to a goal $G$ if $G \subseteq s_{n}$, and the length of $\omega$ is $|\omega|=n$.

A solution is optimal if there does not exists another a better solution, and with better we mean either shorter or lowest total cost (depending on the context). When a solution is not optimal, it is suboptimal. The following definition is very important, especially for unsolvable instances.

Definition 2.3. A state $s$ is reachable with respect to an initial state $I$ if there is a plan from $I$ to $s$. A state is unreachable with respect to $I$ if it is not reachable from $I$. A state $s$ is a dead-end if there is no plan from $s$ to any goal state $g, G \subseteq g$, where $G$ is the goal.

The state transition graph of an instance is an important structure for finding solutions. The nodes of the graph are the total states of the instance, and an edge from one state to another means that there is an applicable action that takes us from the first state to the latter. A path from the initial to a goal state in the graph represents a solution. In other words, one way to solve instances is to use some search algorithm on the state transition graph.

Definition 2.4. Let $\Pi$ be an instance. The set of every total state of $\Pi$ is TotalStates $(\Pi)=\left\{\left\{\left(v_{1}, d_{1}\right), \ldots,\left(v_{n}, d_{n}\right)\right\}: d_{1} \in D_{v_{1}}, \ldots, d_{n} \in\right.$ 
$\left.D_{v_{n}}, n=|V(\Pi)|\right\}$. The state transition graph of $\Pi$ is the directed graph $T G(\Pi)=($ TotalStates $(\Pi), E)$, where $\left(s_{1}, s_{2}\right) \in E$ if and only if there is an action $a \in A(\Pi)$ such that $a$ is applicable in $s_{1}$ and $s_{2}$ is the result of applying $a$ in $s_{1}$.

Example 2.5. We noted in Example 1.2 that the number of total states of $\Pi_{J o e}$ is $2^{6}=64$, i.e. $\mid$ TotalStates $\left(\Pi_{J o e}\right) \mid=64$. The number of goal states is 32 , but not all them are reachable. For example, the state $\left\{\left(\right.\right.$ Joe,$\left.R_{2}\right),(T$, Unsolved $),(K, T),(L$, Locked $),(D$, Closed $),(B$, Eaten $\left.)\right\}$ is unreachable since there is no way for Joe to enter $R_{2}$ without solving T. A plan and solution for $\Pi_{J o e}$ is $\left\langle a_{\text {Solve }}, a_{\text {GrabKey }}, a_{\text {Unlock }}, a_{\text {Open }}\right.$, $\left.a_{R_{1} \rightarrow R_{2}}, a_{\text {Ingest }}\right\rangle$. This is not the only solution; after applying $a_{\text {Open }}$, Joe could $a_{\text {Close }}$ and $a_{\text {Open }}$ again before applying the remainder of the plan. Of course, such a solution is suboptimal (there exists a better solution).

\subsection{Variable Projection}

Some instances have enormous state transition graphs and are very difficult to solve. When this is the case, it might help to relax, or abstract, the instance. Relaxation is a strategy which lets us approximate the solution of an instance by solving an easier, related, instance. There are several ways to relax planning instances, and we focus on variable projection. We define variable projection in the usual way [Hel04].

Definition 2.6. Let $\Pi=(V, A, I, G)$ be a $\mathrm{SAS}^{+}$instance and let $V^{\prime} \subseteq V$. The variable projection of a partial state $s$ onto $V^{\prime}$ is defined as $\left.s\right|_{V^{\prime}}=\left\{(v, d):(v, d) \in s, v \in V^{\prime}\right\}$. The variable projection (or simply projection) of $\Pi$ onto $V^{\prime}$ is $\left.\Pi\right|_{V^{\prime}}=\left(V^{\prime},\left.A\right|_{V^{\prime}},\left.I\right|_{V^{\prime}},\left.G\right|_{V^{\prime}}\right),\left.A\right|_{V^{\prime}}=\left\{\left.a\right|_{V^{\prime}}\right.$ : $\left.a \in A, \operatorname{eff}\left(\left.a\right|_{V^{\prime}}\right) \neq \varnothing\right\}$ where $\operatorname{pre}\left(\left.a\right|_{V^{\prime}}\right)=\left.\operatorname{pre}(a)\right|_{V^{\prime}}$ and $\operatorname{eff}\left(\left.a\right|_{V^{\prime}}\right)=$ $\left.\operatorname{eff}(a)\right|_{V^{\prime}}$.

Note that a variable projection cannot contain actions with an empty effect. It is safe to remove such actions since they cannot be part of any optimal solution, and nor do they affect solvability.

Example 2.7. Suppose $R_{2}$ in Example 1.1 contained an additional 260 bananas and that Joe could not solve the Rubik's Cube since it was rigged. Then it does not matter if we consider that Joe only wants to eat 1 banana, or all 261 of them: the instance is unsolvable in both cases. Hence, it makes sense for Joe to ignore, or project away, all but one banana since if he can devour one banana, then he can devour them all. 
The following theorem is central for this thesis.

Theorem 2.8. [Hel04] Let $\Pi$ be a $\mathrm{SAS}^{+}$planning instance, and $V^{\prime} \subseteq$ $V(\Pi)$. If $\left.\Pi\right|_{V^{\prime}}$ is unsolvable, then $\Pi$ is unsolvable.

Proof. Assume that $\Pi$ is solvable and $\left.\Pi\right|_{V^{\prime}}$ is unsolvable. Let $\omega=$ $\left\langle a_{1}, \ldots, a_{n}\right\rangle$ be a solution for $\Pi$. Let $\left.\omega^{\prime}\right|_{V^{\prime}}=\left\langle\left. a_{1}\right|_{V^{\prime}},\left.a_{2}\right|_{V^{\prime}}, \ldots\right\rangle$ where actions with empty effects have been removed. The actions in $\omega^{\prime}$ are in $\left.A\right|_{V^{\prime}}$ (per definition). The plan $\omega^{\prime}$ is applicable for $\left.\Pi\right|_{V^{\prime}}$ since $\omega$ is applicable for $\Pi$. Furthermore, for every variable $v \in V^{\prime}, v$ is assigned the same values and in the same order. In other words, the final value for every $v$ for $\omega^{\prime}$ is the same as for $\omega$, thus $\omega^{\prime}$ is a solution for $\left.\Pi\right|_{V^{\prime}}$. This contradicts that $\left.\Pi\right|_{V^{\prime}}$ is unsolvable.

Variable projection does not necessarily preserve unsolvability, as the following example demonstrates. That is, if we ignore the 'rigged' Rubik's Cube, then the projection is solvable.

Example 2.9. Suppose we want to project $\Pi_{J o e}$ (see Example 2.1) onto the variable set $\left\{J_{o e}, K, L, D, B\right\}$, i.e. we project away the Rubik's Cube. The domain of every remaining variable is unaffected, but the actions $a_{\text {Solve }}$ and $a_{\text {GrabKey }}$ have changed and their projections are:

- $\left.a_{\text {Solve }}\right|_{\left\{J_{o e}, K, L, D, B\right\}}=\left\{\left(J_{o e}, R_{1}\right)\right\} \rightarrow\{\}$, and

- $\left.a_{\text {GrabKey }}\right|_{\{J o e, K, L, D, B\}}=\left\{\left(J_{o e}, R_{1}\right),(K, T)\right\} \rightarrow\left\{\left(K, J_{o e}\right)\right\}$.

Note that the effect of the first action is empty, i.e. applying it does not change the world state. Clearly, we can remove such actions from the projection. The precondition on $T$ of the second action that required $T$ to be solved has been removed, and Joe can simply grab the key from the non-existing $T$ (a side-effect of projection since there is no 'correct' initial state for the key).

\subsection{Graphs}

The state transition graph is a victim of the combinatorial explosion, and it is often infeasible to represent it in memory. Hence, it is difficult to reason about the instance by using this graph explicitly. Thus, to get a bird's-eye view of how the variables are interconnected it is common to use the causal graph - an important tool that we frequently use. 


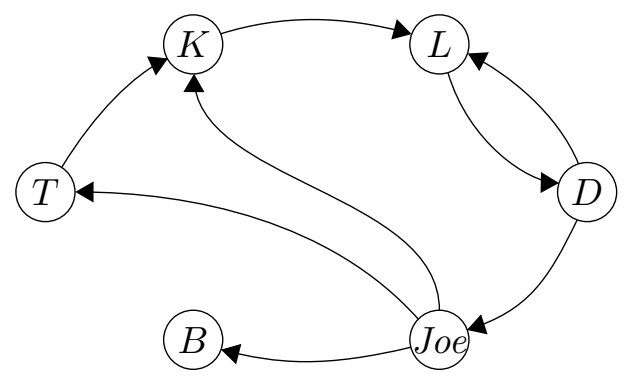

Figure 2.1: The causal graph of $\Pi_{J o e}$ (see Example 2.1).

Definition 2.10. The causal graph $C G(\Pi)$ of a $\mathrm{SAS}^{+}$instance $\Pi=$ $(V, A, I, G)$ is the digraph $(V, E)$ where an edge $\left(v_{1}, v_{2}\right)$, such that $v_{1} \neq v_{2}$, belongs to $E$ if and only if there exists an action $a \in A$ such that $v_{2} \in \operatorname{Domain}(\operatorname{eff}(a))$ and $v_{1} \in \operatorname{Domain}(\operatorname{pre}(a)) \cup \operatorname{Domain}(\operatorname{eff}(a))$.

Example 2.11. Figure 2.1 depicts the causal graph of $\Pi_{J o e}$. A main purpose of the causal graph is to highlight causal relationships between variables. For example, an edge to $B$ from $J o e$ means that a change in state of $B$ depends on the state of Joe. Indeed, $B$ can only be eaten if Joe is in the room.

It is common to define classes of planning instances by imposing restrictions on the causal graph, and many tractable classes has been defined with this approach. We take a much closer look at the causal graph in Part III.

Yet another technique to describe dependencies is the domain transition graph, which detail dependencies between the values of a domain of a variable.

Definition 2.12. The domain transition graph $D T G(v)$ for a variable $v \in V$ is the labelled digraph $\left(D_{v}, E\right)$ where for every distinct $d_{1}, d_{2} \in$ $D_{v},\left(d_{1}, d_{2}\right) \in E$ if and only if there exists an action $a \in A$ such that $\operatorname{pre}(a)[v]=d_{1}$ or $v \notin D o m a i n(\operatorname{pre}(a))$, and $\operatorname{eff}(a)[v]=d_{2}$. The label of an edge is a set of the prevailconditions of the actions that satisfy the requirement for the edge to exist (a set of sets).

Example 2.13. The domain transition graph (DTG) of a variable $v$ illustrates how $v$ can change its state. The $D T G(B)$ of $\Pi_{J o e}$ consists of two nodes and one edge: the nodes Not-eaten and Eaten, and an edge from the former to the latter with the label $\left\{\operatorname{pre}\left(a_{\text {Ingest }}\right) \backslash\{(B\right.$, Not-eaten $\left.)\}\right\}$. We remove non-prevailconditions since they are redundant with the 
direction of the edge. There is no way for $B$ to become Not-eaten once eaten, so there is no edge in that direction.

In some sense, DTGs give a local overview of the planning instance.

\subsection{Computational Problems}

The $\mathrm{SAS}^{+}$planning formalism presented so far have ignored the possibility that actions are not equal, i.e. that there is some kind of 'cost' of applying them. Suppose we want to model this logistics problem: we have a truck, a number of packages and locations where the truck have to deliver the packages to. If we ignore the time it takes for the truck to drive from one location to another, then a solution might be to drive to the location furthest away without delivering any intermediate packages. Clearly, such solutions are suboptimal in practice and to remedy this we can incorporate driving time into the model. One approach is to associate a cost with every action, and in the example the cost is the driving time. In other words, in addition to an instance $\Pi$, we also give a cost function $c: A(\Pi) \rightarrow \mathbb{R}$. The cost of a plan $\omega=\left\langle a_{1}, \ldots, a_{n}\right\rangle$ is $c(\omega)=c\left(a_{1}\right)+\cdots+c\left(a_{n}\right)$.

There are a number of computational problems in planning and the following are the most common ones.

Plan Existence (PE)

InSTANCE: $\mathrm{A} \mathrm{SAS}^{+}$planning instance $\Pi$.

Question: Is there a solution for П?

Bounded Plan Existence (BPE)

Instance: $\mathrm{A} \mathrm{SAS}^{+}$planning instance $\Pi$ and a number $b \in \mathbb{N}$.

Question: Is there a solution $\omega$ for $\Pi$ such that $|\omega| \leq b$ ?

Bounded Cost Plan Existence (BCPE)

InSTANCE: $\mathrm{A} \mathrm{SAS}^{+}$planning instance $\Pi$, a cost function $c: A(\Pi) \rightarrow$ $\mathbb{N}$, and a number $b \in \mathbb{N}$.

QuEstion: Is there a solution $\omega$ for $\Pi$ such that $c(\omega) \leq b$ ?

These three problems are concerned with the existence of a solution, not the generation of one. In some cases, it is easier to verify the existence of a solution than it is to generate one. Furthermore, the problems are sorted in increasing difficulty: PE is easier than BPE since the latter is the optimisation version (if there is a 'short' solution); 
and BPE is easier than BCPE since the former is a special case of the latter, i.e. let the cost of every action be 1 . The following corresponding computational objectives require the generation of a solution.

Plan Generation (PG)

InSTANCE: $\mathrm{A} \mathrm{SAS}^{+}$planning instance $\Pi$.

OBJective: Generate a solution $\omega$ for $\Pi$, if one exists. Otherwise, answer 'no'.

Bounded Plan Generation (BPG)

INSTANCE: $\mathrm{A} \mathrm{SAS}^{+}$planning instance $\Pi$ and a number $b \in \mathbb{N}$.

Oвлестіve: Generate a solution $\omega$ for $\Pi$ such that $|\omega| \leq b$, if one exists. Otherwise, answer 'no'.

Bounded Cost Plan Generation (BCPG)

INSTANCE: $\mathrm{A} \mathrm{SAS}^{+}$planning instance $\Pi$, a cost function $c: A(\Pi) \rightarrow$ $\mathbb{N}$, and a number $b \in \mathbb{N}$.

OBJective: Generate a solution $\omega$ for $\Pi$ such that $c(\omega) \leq b$, if one exists. Otherwise, answer 'no'.

Of course, if we are able to generate a solution, then we can answer the corresponding problem about its existence. If PG does not generate a solution, then no solution exists. However, if BPG or BCPG does not generate a solution then the instance can still be solvable, but a higher bound is required. We also consider the following problems.

\section{Cost Optimal Plan Generation (COPG)}

Instance: $\mathrm{A} \mathrm{SAS}^{+}$planning instance $\Pi$ and a cost function $c$ : $A(\Pi) \rightarrow \mathbb{N}$.

OBJective: Generate a solution $\omega$ for $\Pi$ such that there is no other solution $\omega^{\prime}$ for $\Pi$ where $c\left(\omega^{\prime}\right)<c(\omega)$, if a solution exists. Otherwise, answer 'no'.

Cost Optimal Plan Existence (COPE)

InSTANCE: $\mathrm{A} \mathrm{SAS}^{+}$planning instance $\Pi$ and a cost function $c$ : $A(\Pi) \rightarrow \mathbb{N}$.

OBJective: Generate a cost $c$ such that there is a solution $\omega$ for $\Pi$ where $c(\omega)=c$ and there is no solution $\omega^{\prime}$ for $\Pi$ where $c\left(\omega^{\prime}\right)<c$. If $\Pi$ is unsolvable then $c=\infty$. Otherwise, answer 'no'.

The objective is to generate an optimal solution, or the cost of one, for the instance. The solutions generated when considering BPG and BCPG might not be optimal when the bound $b$ is larger than the cost of an optimal solution. 


\section{Chapter Three \\ Solvable vs. Unsolvable Instances}

We mentioned earlier that we have identified an asymmetry between how much attention solvable and unsolvable instances have gotten in the literature, but is there a theoretical difference between the two? To prove that an instance is, in fact, solvable, we only have to give a solution for the instance. Furthermore, it is straightforward to verify the solution: check if the first action is applicable, and if yes then apply it to the current state. Repeat this for the second action, and so on. If the final state is a goal state then the instance is solvable. In other words, the solution is a certificate that demonstrates solvability. In contrast, it might be more tricky to provide a certificate for unsolvable instances. One certificate is the set of reachable states, and to verify it we need to: (1) check if the initial state is in it; (2) check if no goal state is in it; and (3) check that the set of reachable states is closed under every action. Another possible certificate is the set of states that can reach a goal state. In this case, conditions (1) and (2) are flipped, i.e. the initial state should not be in the set and every goal state should be. The description so far might give the impression that it is computationally easier to verify solvability certificates, however, optimal solutions for solvable instances can be of exponential length and thus expensive to verify. Eriksson, Röger and Helmert [ERH17] describes a family of certificates for unsolvability that can be efficiently verified, and one important aspect of their certificates is a compact representation of states. Another possible certificate is a subset of variables such that the projection onto it is unsolvable, and if the subset is sufficiently small then unsolvability can be verified efficiently. In this chapter we show that there is no theoretical difference between solvable and unsolvable instances. 


\subsection{Exponential Time Hypothesis}

The $k$-SAT problem is NP-complete when $k \geq 3$ [GJ79, problem LO1], and there is no algorithm running in polynomial time if we assume $\mathbf{P} \neq \mathbf{N P}$. However, there is a huge difference between the asymptotic complexity of $\mathcal{O}\left(2^{n}\right)$ and $\mathcal{O}\left(2^{0.386 n}\right)$ - a nuance which is not visible when merely studying complexity classes - but how small can the constant in the exponent be for $k$-SAT? A more precise characterisation of the complexity of $k$-SAT is possible by using the exponential time hypothesis (ETH) [IP01, IPZ01]. This hypothesis is a conjecture stated as follows.

Definition 3.1. For all constant integers $k>2$, let $s_{k}$ be the infimum of all real numbers $\delta$ such that $k$-SAT can be solved in time $\mathcal{O}\left(2^{\delta n}\right)$, where $n$ is the number of variables of an instance. The exponential time hypothesis (ETH) is the conjecture that $s_{k}>0$ for all $k>2$.

Informally, ETH says that satisfiability cannot be solved in subexponential time. One may equivalently define the ETH for the number of clauses, i.e. replacing $\mathcal{O}\left(2^{\delta n}\right)$ with $\mathcal{O}\left(2^{\delta m}\right)$ in Definition 3.1, where $m$ is the number of clauses. It is known that the ETH with respect to the number of clauses holds if and only if the ETH with respect to the number of variables holds [IPZ01]. Note that since the ETH refers to actual deterministic time bounds it is possible to swap the answer, i.e. if we could solve $k$-UNSAT in time $\mathcal{O}(f(n))$ for some function $f$, then we could also solve $k$-SAT in time $\mathcal{O}(f(n))$. Hence, the ETH can equivalently be defined in terms of the $k$-UNSAT problem.

Since using the ETH offers more precision than polynomial reductions between the usual complexity classes do, we will benefit from also being more precise about how much a reduction blows up the instance size, defining the following measure for this purpose.

Definition 3.2. Given a reduction $\rho$ from some problem $X$ to some problem $\mathrm{Y}$, we say that $\rho$ has blow-up $b$, for some $b>0$, if there exists an $n>0$ such that $\|\rho(\boldsymbol{I})\| \leq\|\boldsymbol{I}\|^{b}$ for all instances $\boldsymbol{I}$ of $\mathbf{X}$ such that $\|\boldsymbol{I}\| \geq n$.

Clearly, every polynomial-time reduction has bounded blow-up.

The following properties of 3-CNF formulae will be used in the forthcoming proof. The bounds are straightforward to prove by recalling that we require that $F$ contains no repeated clauses, no empty clauses, and no unused variables. 
Proposition 3.3. Let $F$ be an arbitrary 3-CNF formula with $n$ variables and $m$ clauses. Then one may assume that:

1. $n \leq 3 m$

2. $m \leq 8 n^{3}$,

3. $\|F\| \leq 3 m(1+\log n)$ and

4. $\|F\| \leq 12 m \log m$, for $m \geq 2$.

The time complexity of a problem is usually defined as a function of the instance size, while the ETH is defined as a function of the number of variables or clauses. This latter allows for a sharper characterization but it is not quite suitable for planning where the instance size is not necessarily polynomial in the number of variables. We will instead use time bounds on the form $\mathcal{O}\left(2^{n^{c}}\right)$, where $n$ is the instance size.

Lemma 3.4. 3-SAT and 3-UNSAT cannot be solved in time $\mathcal{O}\left(2^{\|F\|^{c}}\right)$ for any $c<1$, unless the ETH is false.

Proof. Suppose 3-SAT can be solved in time $\mathcal{O}\left(2^{\|F\|^{c}}\right)$ for some $c<1$. We know from Proposition 3.3 that $\|F\| \leq 12 m \log m$, for $m \geq 2$. Furthermore, $12 m \log m<m^{1+\varepsilon}$ for all $\varepsilon>0$ and large $m$. Choose $\varepsilon=1-c$, which satisfies that $\varepsilon>0$ since $c<1$. It then follows from the assumption that 3 -SAT can be solved in time $\mathcal{O}\left(2^{\|F\|^{c}}\right) \subseteq \mathcal{O}\left(2^{m^{(1+\varepsilon) c}}\right)=$ $\mathcal{O}\left(2^{m^{d}}\right)$, where $d=(1+\varepsilon) c=(1+\varepsilon)(1-\varepsilon)<1$. This contradicts the ETH since $2^{m^{d}}$ grows slower than $2^{\delta m}$ for all $\delta>0$. The case for 3-UNSAT is analogous.

\subsection{Solvable vs. Unsolvable Instances}

If we consider a tractable class of planning problems, then it is easy both to find a solution and to find that there is no solution. On the other hand, if we are faced with an NP-hard class, then it cannot be the case that all solvable instances are easy, and only some of the unsolvable instances are hard, as the following result shows.

Theorem 3.5. Let $\mathcal{A}$ be a planning algorithm. Let $C$ be a class of $\mathrm{SAS}^{+}$instances such that $\operatorname{PE}(C)$ is $\mathbf{N P}$-hard and let $\rho$ be a polynomialtime reduction from 3-SAT to $\operatorname{PE}(C)$ with blow-up $b$. If algorithm $\mathcal{A}$ can generate a solution for each solvable instance in $C$ in $2^{\|\Pi\|^{c}}$ steps for some $c$ such that $0<c<\frac{1}{b}$, then the ETH is not true. 
Note that the behaviour of $\mathcal{A}$ only needs to be correct for solvable instances. For unsolvable instances, it may give incorrect answers or not even terminate.

Proof. Suppose there is some algorithm $\mathcal{A}$ that can generate a solution for each solvable instance of $C$ in $2^{\|\Pi\|^{c}}$ steps for some $c$ such that $0<c<1 / b$. Let $F$ be an arbitrary 3-SAT instance and let $\Pi_{F}=\rho(F)$ be the corresponding $\mathrm{SAS}^{+}$instance. Simulate $\mathcal{A}$ on $\Pi_{F}$ for $2^{\left\|\Pi_{F}\right\|^{c}}$ steps. If $\Pi_{F}$ is solvable, then $\mathcal{A}$ will return a correct solution for $\Pi_{F}$, since it has to be correct for all solvable instances. On the other hand, if $\Pi_{F}$ is not solvable, then there are three possibilities:

1. $\mathcal{A}$ answers that $\Pi_{F}$ has no solution,

2. $\mathcal{A}$ does not halt within $2^{\mid\|\Pi\|^{c}}$ steps or

3. $\mathcal{A}$ returns an output string that is not a solution for $\Pi_{F}$.

Obviously, if $\mathcal{A}$ does not return any output string (cases 1 and 2), then $\Pi_{F}$ has no solution. It remains to distinguish between a solvable instance and case 3 . This can be done by checking whether the output string returned by $\mathcal{A}$ is a solution for $\Pi_{F}$ or not. The output string can contain at most $2^{\left\|\Pi_{F}\right\|^{c}}$ symbols, since $\mathcal{A}$ does not have time to produce a longer output string, so there can be at most this many actions in the string. Each step of a solution can be verified in time $p\left(\left\|\Pi_{F}\right\|\right)$ for some fixed polynomial $p$. Hence, the output from $\mathcal{A}$ can be checked in time $\mathcal{O}\left(p\left(\left\|\Pi_{F}\right\|\right) \cdot 2^{\left\|\Pi_{F}\right\|^{c}}\right)$. The construction of $\Pi_{F}$ guarantees that $\mathcal{A}$ outputs a correct solution for $\Pi_{F}$ if and only if $F$ is satisfiable. Hence, we can check whether $F$ is satisfiable or not in time

$$
\mathcal{O}\left(2^{\left\|\Pi_{F}\right\|^{c}}+p\left(\| \Pi_{F}||\right) \cdot 2^{\mid\left\|\Pi_{F}\right\|^{c}}\right) \subseteq \mathcal{O}\left(\left(1+p\left(\| F||^{b}\right)\right) \cdot 2^{\left(\|F\|^{b}\right)^{c}}\right) \subseteq \mathcal{O}\left(2^{\|F\|^{b c+\varepsilon}}\right)
$$

for all $\varepsilon>0$. Furthermore, $b c<1$, since $c<1 / b$, so we can choose $\varepsilon$ such that $0<\varepsilon<1-b c$. However, then $b c+\varepsilon<1$, which contradicts the ETH according to Lemma 3.4.

An immediate consequence of the proof of Theorem 3.5 is the following.

Corollary 3.6. Let $\mathcal{A}$ be a planning algorithm and let $C$ be a class of $\mathrm{SAS}^{+}$instances. If algorithm $\mathcal{A}$ can generate a plan for each solvable instance in $C$ in $f(n)$ steps, then $\operatorname{PE}(C)$ can be solved in $(p(n)+1) \cdot f(n)$ steps for some polynomial $p$ that does not depend on $C$. 
In other words, if $\mathcal{A}$ is a planner that can generate solutions for all solvable instances in $C$, then there exists a planner $\mathcal{A}^{\prime}$ that is sound and complete for $C$ and that has the same time complexity as $\mathcal{A}$ up to a polynomial factor. With this result in mind, one may speculate why planners apparently are better at analysing solvable instances than unsolvable instances in empirical evaluations. One possible explanation is that the development of planners has to a large extent been spurred by the international planning competitions [LCO15]. However, the backside of this development is that these competitions, and thus also most planners, have been heavily focused on instances that are guaranteed to be solvable, that is, the planners and methods used are getting increasingly faster at finding solutions but not on verifying that no solutions exist. Another possible explanation is that the test cases that have been used are not sufficiently large. Corollary 3.6 is an asymptotic result and a planner may very well not behave as expected when given instances that are too small. 



\section{Chapter Four \\ Planners \& Benchmarks}

The methods proposed in this thesis are not implemented in any previously existing planner, but are part of a planner that emerged from this work. Since we compare our implementations with other planners, we need to discuss the most important details of our planner. The chapter is divided into two parts: implementation details of the planner and the benchmarks that we use throughout the thesis to evaluate our proposed methods.

\subsection{Classical Planning Toolkit}

Our planner, Classical Planning Toolkit (CPT), tackles the planning problem the same way as most modern planners. Modern planners often solve planning instances by using some search algorithm in the state transition graph of the instance. Almost all competitors that we compare with are implemented in FAST DOwnward [Hel06a] (FD), which is why the implementation of CPT follows its implementation closely. This is necessary; suppose our planner runs significantly faster than it, then we would like to know if the heuristic function is the reason for the measured difference and not some other implementation detail. The main difference between CPT and FD is the programming language that they are implemented in: $\mathrm{CPT}$ is implemented in $\mathrm{C \#}$, whilst FD is implemented in $\mathrm{C++}$. In most cases, $\mathrm{C}++$ is faster than $\mathrm{C \#}$ by some factor, and we take a brief look at its performance later.

The motivation behind CPT is that the author wanted insight into the design decisions behind modern planners. It should be straightforward to implement the methods presented in this thesis in another planner, such as FD. The programming language $\mathrm{C \#}$ was chosen for its good balance between productivity and performance. 


\subsubsection{Search Algorithms}

Modern planners often search for a path from the initial state to a goal state in the state transition graph of the instance. There is such a path if and only if the instance is solvable, and if such a path is found then it is trivial to derive a solution. The downside is that the size of the state transition graph is exponential in the number of variables, so finding such a path, or deciding if one exists, is very computationally expensive. To deal with the combinational explosion, it is common to use informed search algorithms, such as $\mathrm{A}^{*}$, which are equipped with heuristic functions. When the search algorithm is informed then it can avoid states that either cannot lead to a goal state (a dead-end state); or lead to a costly solution. Therefore, it is able to reduce the number of states that it needs to consider and is thus able to find a solution more quickly. However, whether this actually happens hinges on the heuristic function, which informs the search algorithm.

\section{Heuristic Functions}

In general, a heuristic function is a function that estimates how far away a state is from solving the problem. In planning, a heuristic function often estimates the plan cost of reaching a goal state from a state. There may be a number of applicable actions for a state, all of which might lead to different states, and following up on some of them may have detrimental impact on performance if they are very far away from solving the problem. Hence, an informed search algorithm exploits the heuristic function to avoid actions leading to such states and thus produce a solution more quickly. Throughout this thesis, we are interested in heuristic functions that are:

- Admissible. A heuristic function is admissible if it never overestimates the cost of reaching a goal state.

- Consistent. A heuristic function is consistent if its estimate is always greater than or equal to the estimate for any of its adjacent states, plus the cost of the action to that state.

These two properties are very important for the forthcoming search algorithm. 


\section{A* Search}

The informed search algorithm $\mathrm{A}^{*}$ [HNR68] is a widely used algorithm throughout computer science, and unsurprisingly it is also regularly used in AI. We briefly explain how this algorithm is used when solving planning instances, its properties and present some pseudocode.

Algorithm 4.1 contains pseudocode for an $\mathrm{A}^{*}$ planner. The function $\mathrm{A}^{*}$ takes three arguments: the instance, a heuristic function, and a cost function. Informally, the algorithm starts by generating the immediately reachable states of the initial node, and then use the heuristic function to evaluate them. The states are put in a priority queue, and the priority of a state is the cost it took to reach it plus the estimated cost of reaching a goal state. The algorithm repeats this with the state that has the currently lowest priority until a goal state has been found. If the heuristic function is admissible and consistent, then the algorithm is guaranteed to find an optimal solution (if a solution exists at all). Since this thesis is concerned with solvability, the pseudocode in Algorithm 4.1 does not construct a solution and simply outputs whether the instance is solvable or not.

\section{Depth-first \& Breadth-first Search}

Many of the details of $\mathrm{A}^{*}$ are only useful for solvable instances: if there is no solution then there is no point in considering the cost of actions, prioritising states in the open list, nor trying to estimate the distance to the goal. The only exception to the last point is if the heuristic function can prove that some states are dead-ends (indicated by the heuristic value $\infty$ ). Hence, when dealing with an unsolvable instance, then a simple uninformed search algorithm should perform better. Two such search algorithms are depth-first search [Tré82] (DFS) and breadth-first search [Moo59] (BFS).

Pseudocode for a BFS planner is presented in Algorithm 4.2. Conceptually, the only difference between DFS and BFS is how Open is implemented, and in BFS it is a queue (first in, first out) instead of a stack (last in, first out). In practice, DFS should be implemented recursively to achieve better memory efficiency. We mentioned earlier that heuristic functions that can detect dead-ends are also useful for unsolvable instances, and to add that functionality to these two search algorithms then, just before Line 4 and 13, we evaluate $I$ and $s$, respectively, with the heuristic function to decide whether $s$ is added to Open (when the estimate is not $\infty$ ) or Closed (when the estimate is $\infty$ ). Of 
1 function $\mathrm{A}^{*}(\Pi=(V, A, I, G), h:$ TotalStates $(\Pi) \rightarrow \mathbb{R}$, $c: A \rightarrow \mathbb{R})$

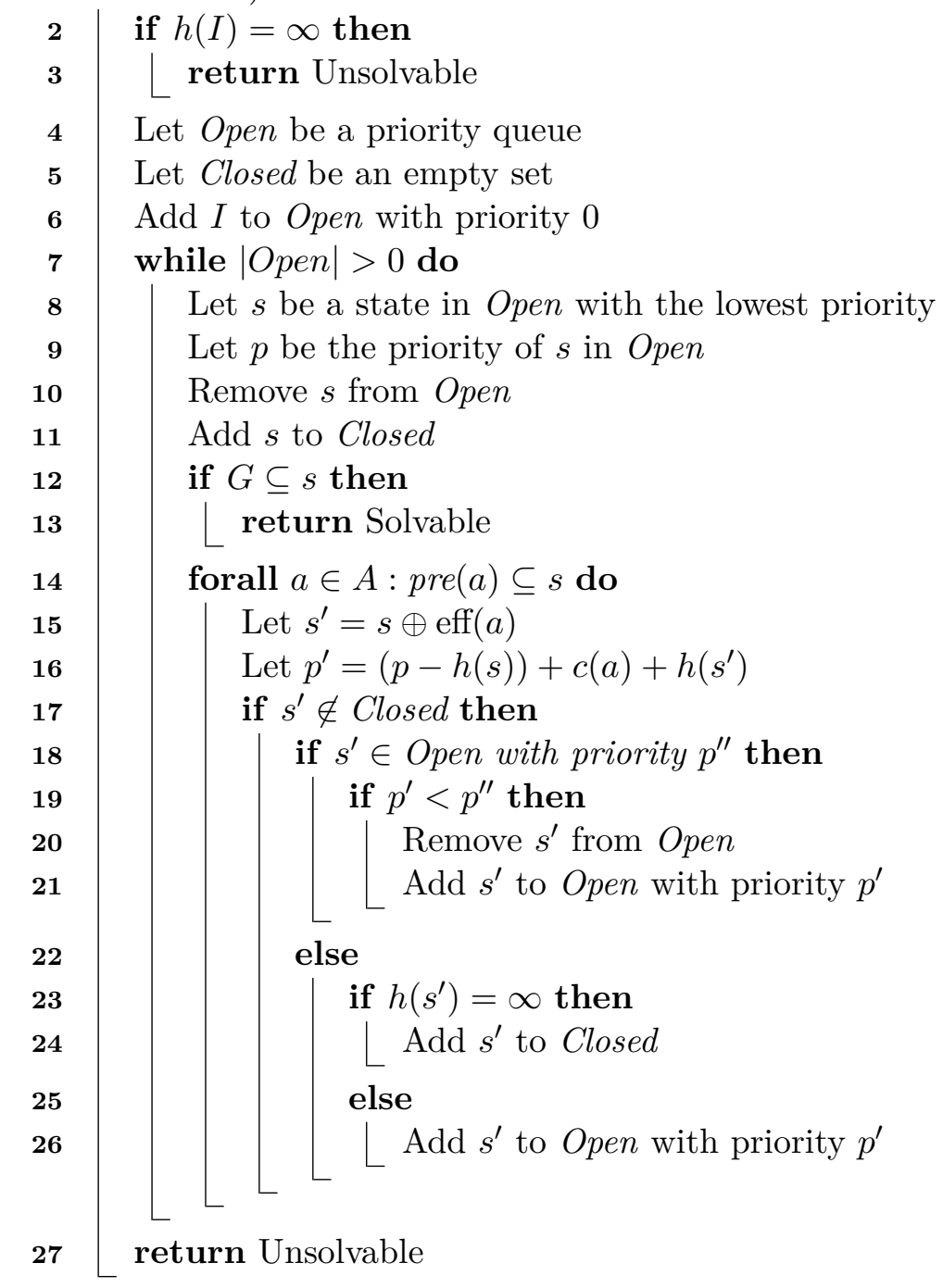

Figure 4.1: Pseudocode for the informed search algorithm A* in the context of $\mathrm{SAS}^{+}$planning. 


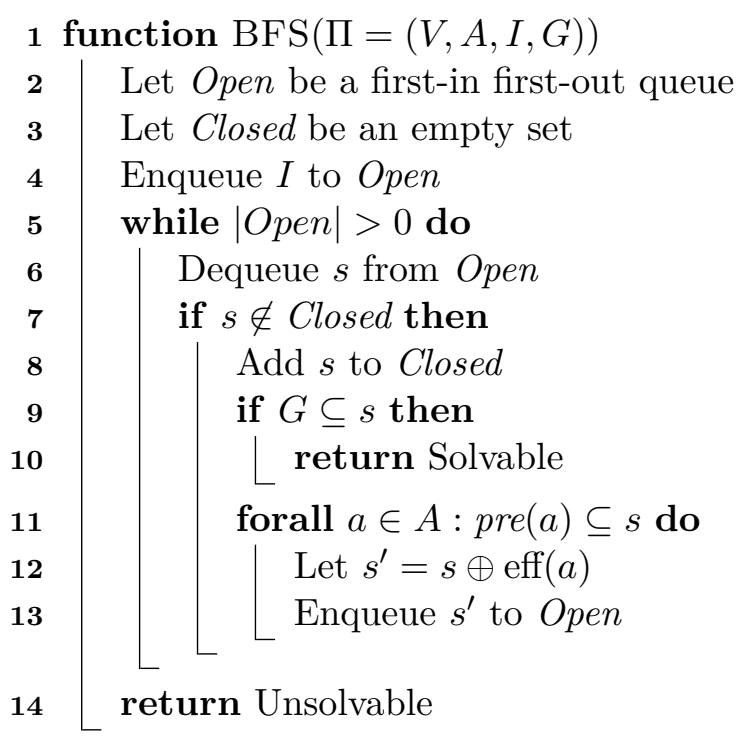

Figure 4.2: Pseudocode for the uninformed search algorithm breadthfirst search in the context of $\mathrm{SAS}^{+}$planning.

course, this only works if the heuristic function is admissible. So far, we have been vague on intricate details such as how a state is represented, how we efficiently find all applicable actions, and how we get the state with the lowest priority.

\section{State Representation}

In Chapter 2 we said that a partial state is a set of variable-value pairs, where every variable occurs in at most one pair. Whilst this is a convenient mathematical representation of a state, it is less suitable for a computer. For performance reasons, it is of outmost importance to represent them well. After all, most of an instance consists of partial states: the initial state, the goal, every state in the state transtion graph, preconditions and effects. These two functions are central to how CPT represents states:

- Let $V_{i d}: V \rightarrow\{0, \ldots,|V|-1\}$ be a bijective function, and

- Let $D_{i d}^{v}: D_{v} \rightarrow\left\{0, \ldots,\left|D_{v}\right|-1\right\}$ be a bijective function.

A total state in CPT is represented as an array $\left\langle n_{0}, \ldots, n_{|V|-1}\right\rangle$, where $\left(D_{i d}^{v}\right)^{-1}\left(n_{i}\right)$ is the value of the variable $\left(V_{i d}\right)^{-1}(i)$ is the state. In other 
words, to construct a CPT total state $s$ we allocate space for an array $a$ of size $|V|$, and for every variable $v \in V$ we write $D_{i d}^{v}(s[v])$ at position $V_{i d}(v)$ in $a$. For partial states, we use some unique natural number $u$ to represent that the variable, at the position in the array, is not defined. i.e. there is no pair with that variable in the mathematical representation. For example, checking whether a precondition matches a state is simply a matter of comparing whether the numbers at the same position, that are not $u$, are equal. This representation is a lot more memory efficient and cache friendly than the mathematical representation.

\section{Open \& Closed List}

CPT stores one copy of every encountered state in a fixed-size block list (a list of fixed size arrays) with time $\mathcal{O}(1)$ for access/indexing (not to be confused with search) and amortised time $\mathcal{O}(1)$ for insert, in the worst case. Note that we never remove states, only add. To figure out exactly where in the block list a state is stored, we need some other data-structure to map states to indices, and we use a hash table which uses the hashing function MurmurHash3 [App16]. The open list contains indices for the block list instead of actual states. Furthermore, information such as whether the state is closed, if its a goal state, the best known plan cost to it, its heuristic value, and so on, is stored together with the state in the block list.

The open list is different in the three search algorithms that we discussed earlier. For DFS and BFS, how it should be implemented is straightforward, however, for informed search algorithms it is less so, and is even an active research subject. A common explanation of $\mathrm{A}^{*}$ is that the open list should be a heap (time $\Theta(1)$ to find a minimum element, time $\Theta(\log n)$ to remove or insert any element, in the worst case), but the range of priorities for planning instances tends to be relatively narrow and heaps exploit this poorly. A more suitable datastructure is to use a hash table (with a good hash function, the expected search and insert time is $\mathcal{O}(1)$ ) where keys are priorities and values are lists of indices. To find an index to a state with a minimum priority, we iterate over the (relatively few) priorities, find the minimum, and then pick some index in the associated list. To find out whether a state is closed, we have to figure out its index in the block list (if it has one). This mapping is also done with the aforementioned hash table where keys are states and values are indices, so deciding whether we have seen a state before should be fast. 


\begin{tabular}{lrrrr}
\hline & Bottleneck & Pegsol & $3-S A T$ & TPP \\
& $6-2$ & 30 & $65-15-1$ & $05-02$ \\
\hline $\mathrm{CPT}$ & $25 \mathrm{~s}$ & $13 \mathrm{~s}$ & $112 \mathrm{~s}$ & $32 \mathrm{~s}$ \\
FD & $37 \mathrm{~s}$ & $15 \mathrm{~s}$ & $62 \mathrm{~s}$ & $35 \mathrm{~s}$ \\
\hline
\end{tabular}

Table 4.1: The total real time it took for CPT and FD to visit every reachable state (rounded to closest full second), for 4 instances in 4 different domains. The numbers below the domain name is the specific instance in the benchmarks. Both planners were configured to use $\mathrm{A}^{*}$ with a blind heuristic function.

\section{Applicable Actions}

It is common that only a few actions are applicable in any state, and therefore iterating over the list of every action to check if it is applicable is wasteful. CPT generates a list of applicable actions the same way as FD does: by traversing a decision tree, where nodes are variables, the branches are values, and leaf nodes are lists of actions. A path from the root to a leaf node determines which actions are in the list, more specifically, the path must have assigned a single value, including the undefined value $u$, to every variable and they are the preconditions of the actions in the list. Thus, generating a list of applicable actions is simply a matter of traversing the decision tree, by chosing branches marked with the value of the variable in the state and $u$ (to get actions with no precondition on the variable). We refer the reader to Helmert [Hel06a] for an in-depth explanation.

\subsubsection{Performance Comparison}

We also carried out a small performance comparison between CPT and FD, which is presented in Table 4.1. To make the comparison fair, we gave the planners unsolvable instances and they use the same configuration. The configuration is $\mathrm{A}^{*}$ with a blind heuristic function (which returns 1 if the state is not a goal state, 0 otherwise). Note that, the order in which the planners visit the reachable states does not matter since the instances are unsolvable, so the benchmarks measure raw performance: time spent per state. Both planners were run on an Intel Core i7-6700K at $4.00 \mathrm{GHz}$ with $32 \mathrm{~GB}$ RAM, and on Windows 10. CPT ran using .NET Core, and FD was (natively) compiled using MinGW64. 
The instances in Table 4.1 are explained in the next section. The results in Table 4.1 are surprising: CPT was able to beat FD in 3 out of 4 instances. This is unexpected since C++ is normally a faster language than $\mathrm{C \#}$. One of the features of $\mathrm{C \#}$ is that memory is managed with the help of a garbage collector, which also runs on a different thread. Thanks to managed memory, allocations and deallocations of memory are very fast, and these two are performance bottlenecks in CPT (and perhaps also in FD). Since the garbage collector runs on a different thread, the time spent managing memory is not included in the measured time so CPT sometimes runs faster than FD. The reason why CPT performs badly on 3-SAT is because of how it represents states: it always allocate 16 bits of memory per binary variable, instead of 1 bit. Consequently, memory operations such as copying states and applying actions are slower. FD is more memory efficient when it comes to this, and for some instances CPT runs out of memory whilst FD does not.

Ultimately, the performance of CPT and FD is comparable, but raw performance is not imperative to begin with: a good heuristic function can easily improve these planners by an order of magnitude by reducing the number of states that they have to consider.

\subsection{Benchmarks of Unsolvable Instances}

Throughout this thesis, the following domains are repeatedly discussed and statistically analysed. The instances were provided by Hoffman et al. [HKT14], and we give a brief explanation of the domains in the portfolio:

- 3-SAT: We introduced $k$-SAT in Chapter 1.2, and unsolvable formulas are taken from the phase transition region. The phase transition region is, intuitively, when there are not too few and not too many clauses in a formula, and such formulas are regarded as being hard to solve [GW94].

- Bottleneck: $n$ agents have to move to individual goals on a grid of tiles. However, when an agent moves to a tile then the tile is flipped, and agents can not move to a flipped tile. Instances are unsolvable since only $n-1$ agents can fulfill their goal.

- Mystery: A fuel-restricted transportation domain where the names of different types are obscured (e.g., locations are named foods). 
The fuel is a property of every location, and there is not enough fuel to achieve the goal.

- No mystery: Same as Mystery, but the names are not obfuscated and the fuel is a property of every truck.

- Peg Solitaire (Pegsol): A tabletop game where, for these instances, the (impossible) goal is to have a single peg in the centre of the board.

- Rovers: A power-restricted rover which has to complete its mission before it runs out of energy. Normally, the rover is able to recharge but not in these instances, making them unsolvable.

- TPP: An agent is given a budget and can buy goods, drive between markets with different prices, and sell goods. The goal is to own a lot of goods, but its impossible to earn enough money to own enough at the same time.

- Tiles: A sliding tiles puzzle where the initial state of the puzzle is taken from an unsolvable part of the state transition graph.

The results in this thesis are presented in chronological order and to make the experiments tell a story, we do not compare the results with results for planners that were developed later. Furthermore, we do not use every benchmark in some experiments for the same reason.

There are more benchmarks available. However, some of the statistical analyses that we carried out were very computationally expensive (we used a large computer cluster), and the computations were performed before many of them were available. For this reason, we use somewhat restricted set of benchmarks throughout this thesis. This does not change any conclusions. 



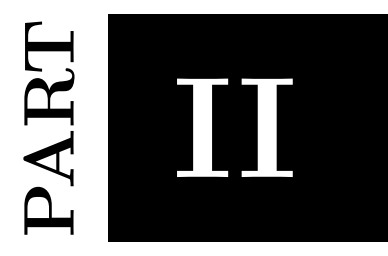

Variable Projection 


\section{Overview of Part II}

In Chapter 2 we formally defined variable projection and gave some examples. The larger part of this thesis studies variable projection with the goal of using it to show unsolvability. Our first method is based on Theorem 2.8, which states that if a projection is unsolvable, then so is the original instance. Observe that Theorem 2.8 only works in one direction, and there is no guarantee that there exists a proper subset $V^{\prime} \subset V$ which proves unsolvability. Nevertheless, this theorem is the basis for our first method, consistency checking, which is presented in Chapter 5.

Chapter 6 strays away from the ambition of finding a single unsolvable projection, and instead attempts to find projections that provide good dead-end detection. This is often easier in practice since we do not restrict ourselves to proving that a single state, i.e. the initial state, is a dead-end. The identified dead-ends are then used to combat the combinatorial explosion when searching for a solution for the original instance. 


\section{Chapter Five \\ Consistency Checking}

The forthcoming method for proving unsolvability, which is presented in Section 5.1, balances completeness against efficiency through a parameter $k$ : the algorithm identifies more unsolvable instances but takes more time for increasing values of $k$. The algorithm runs in polynomial time with respect to the instance size, when $k$ is fixed. In Section 5.2 we analyse consistency checking formally with respect to computational complexity, and in Section 5.3 we take a more hands-on approach and try to solve every projection at the two lowest inconsistent levels, for every benchmark, to get a picture of how viable consistency checking is in practice. Finally, in Section 5.4 we compare its performance to planners that existed when the method was published, on a subset of the benchmarks.

\subsection{Definitions}

The cornerstone of consistency checking is Theorem 2.8: if the projection of an instance onto a subset of its variables is unsolvable, then the original instance is also unsolvable. To show that an instance $\Pi$ is unsolvable, it is thus sufficient to project the instance onto a single subset $V^{\prime} \subseteq V(\Pi)$ such that the projection $\left.\Pi\right|_{V^{\prime}}$ is unsolvable. This is a very powerful fact, but one that comes at a cost; deciding if such a subset exists is obviously as hard as deciding if $\Pi$ is solvable. In order to gain any efficiency at all from this idea, we must thus necessarily settle for an incomplete method. Based on the assumption that there will often exist a small subset of variables that suffices as a witness for unsolvability, the basic principle of our method is to check all subsets of variables up to some predefined size $k$. Since, as we will see, many planners perform badly on unsolvable instances, an obvious use of this method is as a preprocessing step, attempting to decide if the instance 
is unsolvable before even invoking the planner to search for a solution. The parameter $k$ can be used to balance time against coverage; a higher value of $k$ requires more time but also catches more unsolvable instances.

Definition 5.1. [BJS13] Let $\Pi$ be a $\mathrm{SAS}^{+}$instance and $1 \leq k \leq V(\Pi)$ be an integer. Then level $k$ of $\Pi$ is the set $\left\{\left.\Pi\right|_{V^{\prime}}: V^{\prime} \subseteq V(\Pi),\left|V^{\prime}\right|=k\right\}$. We say that a level is consistent if every projection in it is solvable, and inconsistent otherwise. A SAS ${ }^{+}$instance $\Pi$ is variable $k$-consistent for an integer $k$ if level $k$ of $\Pi$ is consistent.

The two definitions are similar and the main difference is that we can refer to a specific set of projections when talking about levels, which is sometimes useful. We very often write $k$-(in)consistent instead of variable $k$-(in)consistent. We define the computational problem in the following way.

\section{Consistency Checking}

Instance: $\mathrm{A} \mathrm{SAS}^{+}$instance $\Pi$ and an integer $1 \leq k \leq|V(\Pi)|$.

Question: Is $\Pi$ variable $k$-consistent?

Clearly, the number of subsets to check grows quickly with the value of $k$. If there are $n$ variables in total, then there are $\left(\begin{array}{l}n \\ k\end{array}\right)$ subsets of size $k$, so checking consistency is usually only feasible for small values of $k$. If an instance is consistent for a particular $k$, then it is also consistent for all $k^{\prime}<k$ by definition, so if we have decided on a fixed $k$ to check consistency for, it is sufficient to check all subsets exactly of size $k$. In practice, however, it may be desirable to make an iterative computation that checks also all smaller sets in order of increasing size, since the algorithm may often terminate early, for some set of smaller size than $k$. Similarly, if an instance is inconsistent for a particular $k$, then it is also inconsistent for all $k^{\prime}>k$. Furthermore, if we do not fix $k$ in advance, then we can check consistency for increasing values of $k$ until the algorithm is stopped or hits a resource limit. The longer it runs, the greater the chance that it will detect that an unsolvable instance is unsolvable.

The straightforward iterative algorithm appears in Figure 5.1. The test in Line 4 can be performed by any complete and sound planner. Of course, our implementation uses CPT and in this version of consistency checking, CPT was configured to use depth-first search. The algorithm is reasonably efficient for small values of $k$, but it can easily be improved by using well-known and obvious techniques. We will consider the following two straightforward methods for filtering which subsets to check (Line 3). 
1 function ConsistencyCheck $(\Pi, k)$

\begin{tabular}{l|c}
$\mathbf{2}$ & for $i=1$ to $k$ do \\
$\mathbf{3}$ \\
$\mathbf{5}$
\end{tabular} \mid $\begin{array}{r}\text { forall } V^{\prime} \subseteq V(\Pi),\left|V^{\prime}\right|=i \text { do } \\
\text { if }\left.\Pi\right|_{V^{\prime}} \text { is unsolvable then } \\
\text { return } k \text {-inconsistent }\end{array}$

Figure 5.1: The consistency checking method by Bäckström et al. [BJS13].

1. A projection without any goal variables is solved by the empty plan, so it is sufficient to check projections containing at least one goal variable.

2. A variable projection induces a subgraph of the causal graph which needs not be weakly connected even if the causal graph is. The components of the subgraph define independent subinstances, so if a projection to $k$ variables induces more than one component, it suffices to check consistency for each component separately. In the incremental case we need not even do that; every component that is smaller than $k$ must already have been checked in an earlier iteration, so it is not necessary to check consistency for the projection at all.

As a further improvement, our implementation traverses the causal graph to generate only those sets that are connected, thus avoiding to even consider the non-connected ones. Suppose we have a weakly connected component $C$ of size $k$, then we can generate a weakly connected component $C^{\prime}$ of size $k+1$ by adding a variable $v \notin C$ such that $\left(v, v^{\prime}\right)$ or $\left(v^{\prime}, v\right)$ is an edge in the causal graph, where $v^{\prime} \in C$. This is done for every possible $v$, not just one. If we do this for every component of size $k$ that satisfies (1) and (2), then we get every component of size $k+1$ that satisfies (1) and (2). Of course, a component might be generated several times and we must check if it has been generated already. The initial components are each individual goal variable. 


\subsection{Complexity Analysis}

We start by analysing Consistency Checking with standard complexity theory and show that it can be decided in polynomial time for fixed integers $k$.

Theorem 5.2. The problem Consistency Checking can be answered in time $\mathcal{O}\left(n^{k} \cdot d^{3 k}\right)$, for every $k$, where $n$ is the number of variables and $d$ is the maximum domain size.

Proof. Let $\langle\Pi, k\rangle$ be an instance of Consistency Checking of size $n$ with $m$ variables of maximum domain size $d$. Then $m \leq n$ and $d \leq n$ by the standard assumption of reasonable encodings [GJ79]. There are $\left(\begin{array}{c}m \\ k\end{array}\right)=\frac{m !}{(m-k) ! k !}<\frac{(m-k) ! m^{k}}{(m-k) ! k !}=\frac{1}{k !} m^{k}$ subsets of size $k$. Each projected instance has a state space with $\leq d^{k}$ states, and checking if it is solvable can be done by Dijkstra's algorithm in $\mathcal{O}\left(\left(d^{k}\right)^{3}\right)=\mathcal{O}\left(d^{3 k}\right)$ time. We get a grand total of $\mathcal{O}\left(m^{k} d^{3 k}\right)$ time, i.e. $\mathcal{O}\left(n^{4 k}\right)$ time, for checking variable $k$-consistency for $\Pi$.

Although $k$-consistency can be checked in polynomial time for every fixed $k$, it is problematic that the degree of the polynomial grows with the constant $k$. However, it could still be the case that we could solve the problem in a way that does not scale as badly with the value of $k$ by using some other algorithm.

A way to analyse that possibility is to use parameterised complexity theory. We begin by defining the basic notions of parameterised complexity theory and refer to other sources [DF99, FG06] for an in-depth treatment. A parameterised problem is a set of instances $\langle\boldsymbol{I}, k\rangle$, where $\boldsymbol{I}$ is the main part and $k$ the parameter (usually a non-negative integer). The problem is fixed-parameter tractable (FPT) if there is an algorithm that solves any instance $\langle\boldsymbol{I}, k\rangle$ of size $n$ in time $f(k) \cdot n^{c}$, where $f$ is an arbitrary computable function and $c$ is a constant independent of both $n$ and $k$. Note that this expression is separable in the sense that $f(k)$ must not depend on $n$ and the polynomial $n^{c}$ must not depend on $k$. FPT is the class of all fixed-parameter tractable decision problems. There are also various classes used to prove that problems are hard, eg. the class $\mathbf{W}[1]$.

Parameterised complexity offers a completeness theory, similar to the theory of NP-completeness, that allows the accumulation of strong theoretical evidence that some parameterised problems are not fixedparameter tractable. This theory is based on a hierarchy of complexity 
classes

$$
\mathbf{F P T} \subseteq \mathbf{W}[1] \subseteq \mathbf{W}[2] \subseteq \mathbf{W}[3] \subseteq \cdots
$$

where all inclusions are believed to be strict. Each class $\mathbf{W}[i]$ contains all parameterised decision problems that can be reduced to a certain canonical parameterised problem under parameterised reductions. A parameterised problem $L$ reduces to a parameterised problem $L^{\prime}$ if there is a mapping $R$ from instances of $L$ to instances of $L^{\prime}$ such that:

1. $\langle\boldsymbol{I}, k\rangle$ is a Yes-instance of $L$ if and only if $\left\langle\boldsymbol{I}^{\prime}, k^{\prime}\right\rangle=R(\boldsymbol{I}, k)$ is a YES-instance of $L^{\prime}$,

2. There is a computable function $f$ and a constant $c$ such that $R$ can be computed in time $\mathcal{O}\left(f(k) \cdot n^{c}\right)$, where $n$ denotes the size of $\langle\boldsymbol{I}, k\rangle$, and

3. There is a computable function $g$ such that $k^{\prime} \leq g(k)$.

Not much is known about the relationship between the parameterised complexity classes and the standard ones, except that $\mathbf{P} \subseteq$ FPT.

If we could prove that Consistency Checking is in FPT, then we would know that there is an algorithm that might still scale badly in $k$ but not in the combination of $k$ and $n$ as the polynomial $n^{k}$ does. Unfortunately, if we consider $k$ as a parameter there is little hope for such an algorithm, since the problem can be shown to be hard for the class $\mathbf{W}[1]$.

Theorem 5.3. Consistency Checking is $\mathbf{W}[1]$-hard when parameterised by $k$.

Proof. We present a parameterised reduction from Independent Set, which is $\mathbf{W}$ [1]-hard [DF99], to Consistency Checking. Let $(V, E)$ and $k \geq 0$ denote an arbitrary instance of Independent Set and assume $V=\left\{v_{1}, \ldots, v_{n}\right\}$. Construct a planning instance $\Pi=(W, A, I, G)$ as follows. Define the actions $b_{i}^{j}:\left\{\left(w_{i}, s\right)\right\} \rightarrow\left\{\left(w_{i}, v_{j}\right)\right\}$ and $c_{i j}^{l m}$ : $\left\{\left(e_{i j}, 0\right),\left(w_{i}, v_{l}\right),\left(w_{j}, v_{m}\right)\right\} \rightarrow\left\{\left(e_{i j}, 1\right)\right\}$, and let

- $W=\left\{w_{1}, \ldots, w_{k}\right\} \cup\left\{e_{i j}: 1 \leq i<j \leq k\right\}$

- $D\left(w_{i}\right)=\left\{s, v_{1}, \ldots, v_{n}\right\}, 1 \leq i \leq k$

- $D\left(e_{i j}\right)=\{0,1\}, 1 \leq i<j \leq k$

- $A=\left\{b_{i}^{j}: 1 \leq i \leq k, 1 \leq j \leq n\right\} \cup\left\{c_{i j}^{l m}: 1 \leq i<j \leq k, 1 \leq\right.$ $\left.l, m \leq n, l \neq m,\left(v_{l}, v_{m}\right) \notin E\right\}$, 
- $I=\left\{\left(w_{i}, s\right): 1 \leq i \leq k\right\} \cup\left\{\left(e_{i j}, 0\right): 1 \leq i<j \leq k\right\}$

- $G=\left\{\left(e_{i j}, 1\right): 1 \leq i<j \leq k\right\}$

To prove that this is indeed a parameterised reduction, we first prove that $\Pi$ has a solution if and only if $G$ has an independent set of size $k$. Assume $G$ contains an independent set $\left\{v_{1}, \ldots, v_{k}\right\}$. Then, the following is a valid solution for $\Pi$ for some function $f:[1, k] \rightarrow[1, n]$ that tells how the $b_{i}^{j}$ actions select the $k$ independent vertices from $V$ :

$$
b_{1}^{f(1)}, \ldots, b_{k}^{f(k)}, c_{11}^{f(1) f(1)}, c_{12}^{f(1) f(2)}, \ldots, c_{(k-1) k}^{f(k-1) f(k)} .
$$

Assume instead that $\Pi$ has a valid solution $\omega$. Since all variables $e_{i j}$ are set to 1 after the execution of $\omega$, it follows that all variables $w_{1}, \ldots, w_{k}$ are given distinct values. Additionally, these values (if interpreted as vertices in the original graph) form an independent set. Now note that $|W|=k+k(k-1)=k^{2}$ so $\Pi$ has a solution if and only if $\Pi$ is variable $k^{2}$-consistent. Thus, it satisfies condition (1) and (3). To see that it satisfies condition (2), it is sufficient to note that the reduction can be performed in $\mathcal{O}(\operatorname{poly}(k) \cdot \operatorname{poly}(|V|+|E|))$ time: we introduce $k+k(k-1)$ variables where each variables has a domain of size $\leq n+1$ and we introduce less than $k \cdot n+k^{2} \cdot n^{2}$ actions.

Despite this negative result, Consistency Checking could still be in FPT for other parameters.

\subsection{Statistical Analysis}

When we introduced consistency checking, we said that it was based on the assumption that there often exists a small subset of variables that suffices as a witness for unsolvability. The statistical analyses in this section investigate precisely this assumption, and whether there often are similarities between such witnesses. The statistical data details how many unsolvable projections there are, and if the unsolvable projections share a significant number of variables. We also look into whether unsolvable projections tend to form clusters with respect to how many variables they have in common, and it turns out that they do. In Chapter 8 we take an even closer look at unsolvable projections and not only consider how many variables they share, but also the structure of their domain transition graph and how they influence one another in the causal graph. 


\begin{tabular}{lrcrrrr}
\hline & & \multicolumn{2}{c}{ \% of unsolvable projections } & & \multicolumn{2}{c}{ \# of instances } \\
Domain (\#) & Avg. $k$ & Level $k$ & Level $k+1$ & & Level $k$ & Level $k+1$ \\
\hline 3-SAT (30) & 11 & 0.002 & - & 5 & 0 \\
Bottleneck (25) & 4 & 1.425 & 3.755 & 20 & 20 \\
Mystery (9) & 3.63 & 9.008 & 12.434 & 8 & 8 \\
No Mystery (25) & 4.72 & 1.646 & 7.917 & 25 & 20 \\
Pegsol (24) & 10.90 & 0.176 & 0.195 & 21 & 2 \\
Rovers (25) & 4 & 0.002 & 0.005 & 3 & 3 \\
TPP (25) & 4.2 & 0.039 & 0.152 & 5 & 4 \\
\hline
\end{tabular}

Table 5.1: The average ratio between the number of unsolvable projections and the total number of projections for the lowest inconsistent level $k$, and if possible level $k+1$, for every domain. However, it was not computationally feasible to solve every projection in both levels for every instance (especially 3-SAT). We also give the average lowest inconsistent level (avg. $k$ ). The last two columns detail for how many instances we were able to do this. The Tiles domain is omitted since every projection that we considered was solvable, i.e. variable $k$-inconsistency can only be found for a large $k$.

The data used in the forthcoming analysis was collected by a single computer, which solved a very large number of projections during the course of several months, starting with projections with the fewest variables. Every projection had a weakly connected causal graph and at least one goal variable. Of course, for most instances, there are simply too many projections to solve each and every one so the data is by necessity incomplete.

\subsubsection{Unsolvable Projections}

We will now ask questions such as: What is the lowest value of $k$ for which level $k$ is inconsistent? What is the ratio between the number of unsolvable projection and the total number of projections? Is this ratio higher at level $k+1$ ? The ratio can decrease for higher levels since we require projections to have a weakly connected causal graph. Table 5.1 presents data to answer these questions. The average ratios between unsolvable and solvable projections in Table 5.1 are for the lowest inconsistent level $k$ and the next level, $k+1$. We observed that the ratios did not vary much between instances of the same domain. Unsurprisingly, the overall ratios are low at level $k$ : 3-SAT and Rovers had the lowest average ratio of about $0.002 \%$; Mystery had the highest 
average ratio of about $9 \%$; and the average ratio (of the averages) over every domain was about $1.76 \%$. At level $k+1$, the situation improved substantially: Rovers had the lowest ratio with about $0.005 \%$ unsolvable projections, which is almost 3 times more than the previous level; and the highest ratio was about $12.43 \%$ for the Mystery domain. The average ratio (of the averages) over every domain was $4.08 \%$ - slightly higher than 2 times the previous level. The outlier is Pegsol, whose ratio barely increased from level $k$ to level $k+1$, and we will shed some light on why this is the case later on.

The good news is that, for most domains, the ratio increases and later on we will see that we can expect the ratio to increase at higher levels. It is important to know how many unsolvable projections there are in order to verify that the forthcoming histograms observe something interesting. For example, if the ratio of unsolvable and solvable 'neighbours' is higher than the overall ratio of unsolvable and solvable projections, then unsolvable projections tend to be 'neighbours'.

\subsubsection{Neighbour Projections}

We conjecture that unsolvable projections tend to have a lot of variables in common. Intuitively, the (impossible) dependencies between some variables is why the instance is unsolvable, and unsolvable projections are able to isolate these dependencies.

\section{- Note}

A histogram is a graphical representation of the frequency of the given data points, that is, the more frequent a data point is, the taller its corresponding bar is. For instance, a tall bar at $0 \%$ means that many projections have around $0 \%$ unsolvable neighbours. In the upcoming histograms, we have aggregated the result for every instance in the same domain. For example, suppose $\Pi_{1}, \Pi_{2}$ and $\Pi_{3}$ are part of the same domain $\mathcal{D}$, and the lowest inconsistent level for $\Pi_{i}$ is $L_{i}$. Let $U=\bigcup_{j=1}^{3}\left\{\left.\Pi_{j}\right|_{V}:\left.\Pi_{j}\right|_{V}\right.$ is an unsolvable projection in level $\left.L_{j}\right\}$. Then the data of the histogram is based on $U$, e.g. the percentage of neighbours every projection in $U$ has. We do this since presenting 183 histograms is impractical. Luckily, histograms for instances within the same domain tend to have a similar distribution. 
Definition 5.4. Let $\left.\Pi\right|_{V_{1}}$ and $\left.\Pi\right|_{V_{2}}$ be projections that both have weakly connected causal graphs and contain at least one goal variable each. Then $\left.\Pi\right|_{V_{1}}$ and $\left.\Pi\right|_{V_{2}}$ are neighbours if $\left|V_{1}\right|=\left|V_{2}\right|$ and $\left|V_{1} \cap V_{2}\right|=\left|V_{1}\right|-1$.

In other words, two neighbours share all but one variable and we examine whether most unsolvable projections are also neighbours. Figure 5.2 and 5.3 present histograms over how many unsolvable neighbours a projection have at the lowest inconsistent level $k$ and the next level $(k+1)$, respectively. The difference between the two figures is that Figure 5.2 is restricted to unsolvable projections, whilst Figure 5.3 is not. The massive difference between these two figures implies that unsolvable projections tend to have many variables in common. For example, unsolvable projections at level $k$ for Pegsol has an average of $14.2 \%$ unsolvable neighbours, whilst the same figure for all projections is $0.2 \%$. In other words, an unsolvable projection is much more likely to have unsolvable neighbours than a solvable projection. The average ratio (of the averages) between unsolvable and solvable neighbours for unsolvable projections was about $7.86 \%$ and $32.85 \%$ for level $k$ and $k+1$, respectively. These ratios are significantly higher than the corresponding averages for all projections $(0.17 \%$ and $1.3 \%)$. When the ratios differ this significantly, then we are able to reason about how likely a projection is to be solvable by looking at its neighbours: if we know that all of its neighbours are solvable, then the projection is very likely to be solvable as well.

A natural follow-up question is what happens if we allow neighbours to differ in more than one variable.

Definition 5.5. A neighbourhood of unsolvable projections is the transitive closure of the neighbour relation on unsolvable neighbours.

In other words, every projection in the neighbourhood is unsolvable. Intuitively, a neighbourhood is a set of projections that have many variables in common. Table 5.2 details how many neighbourhoods there are and how large they are. For every domain, except Pegsol, the number of neighbourhoods are very few and they can be quite large, especially at level $k+1$. The outlier is still Pegsol, where the histogram for level $k$ and level $k+1$ are about the same, and they have about the same average. For TPP, we note that, even though the unsolvable projections at level $k$ have $0 \%$ neighbours, there is on the average only one neighbourhood (of average size 1). The number of neighbourhoods can go down from one level to another if they merge, and this happens 


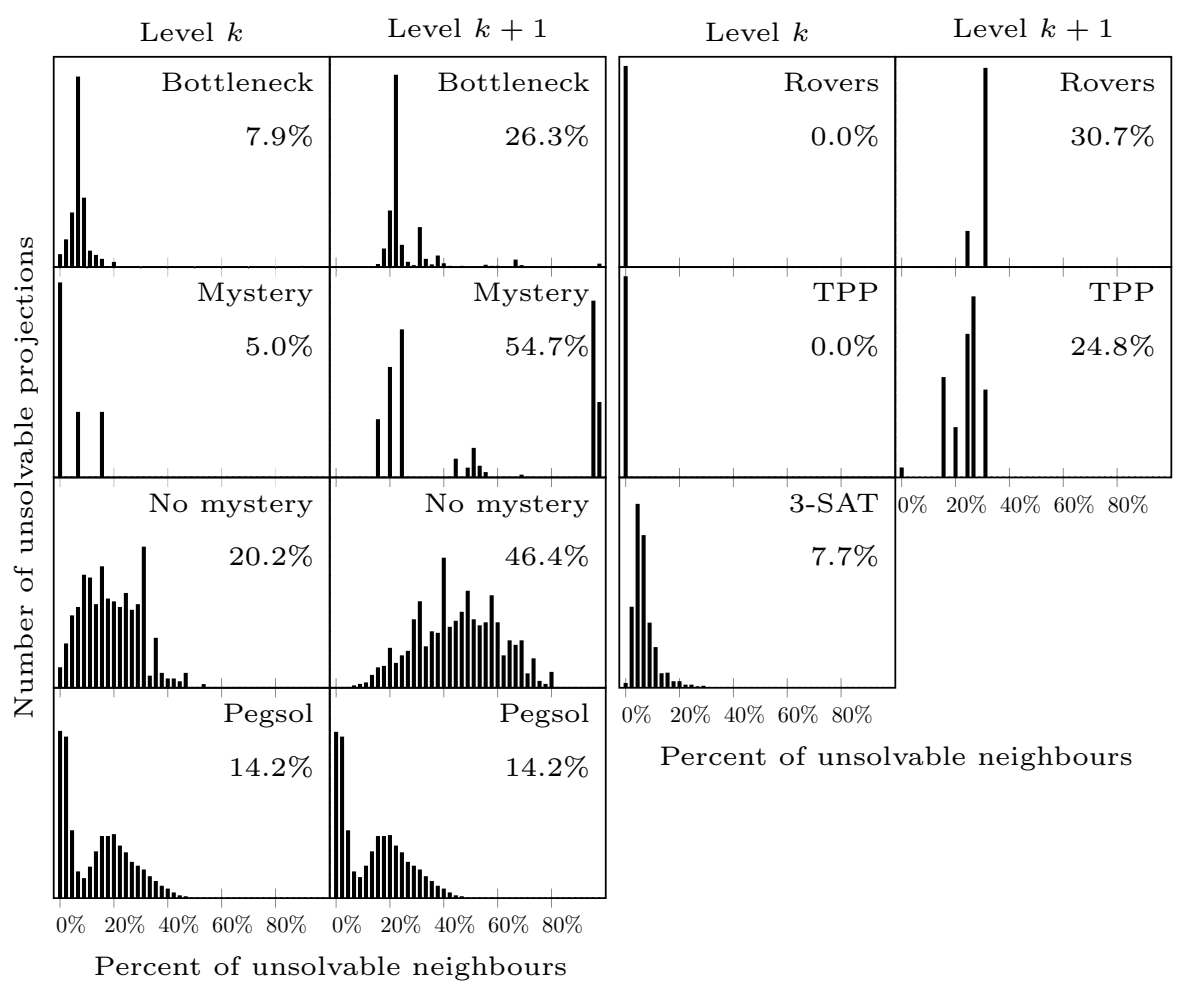

Figure 5.2: Histograms of the ratio between the number of unsolvable neighbours and the total number of neighbours that unsolvable projections have. The histograms to the left are for the lowest inconsistent level $k$, and the corresponding histograms to the right are for the next level. 


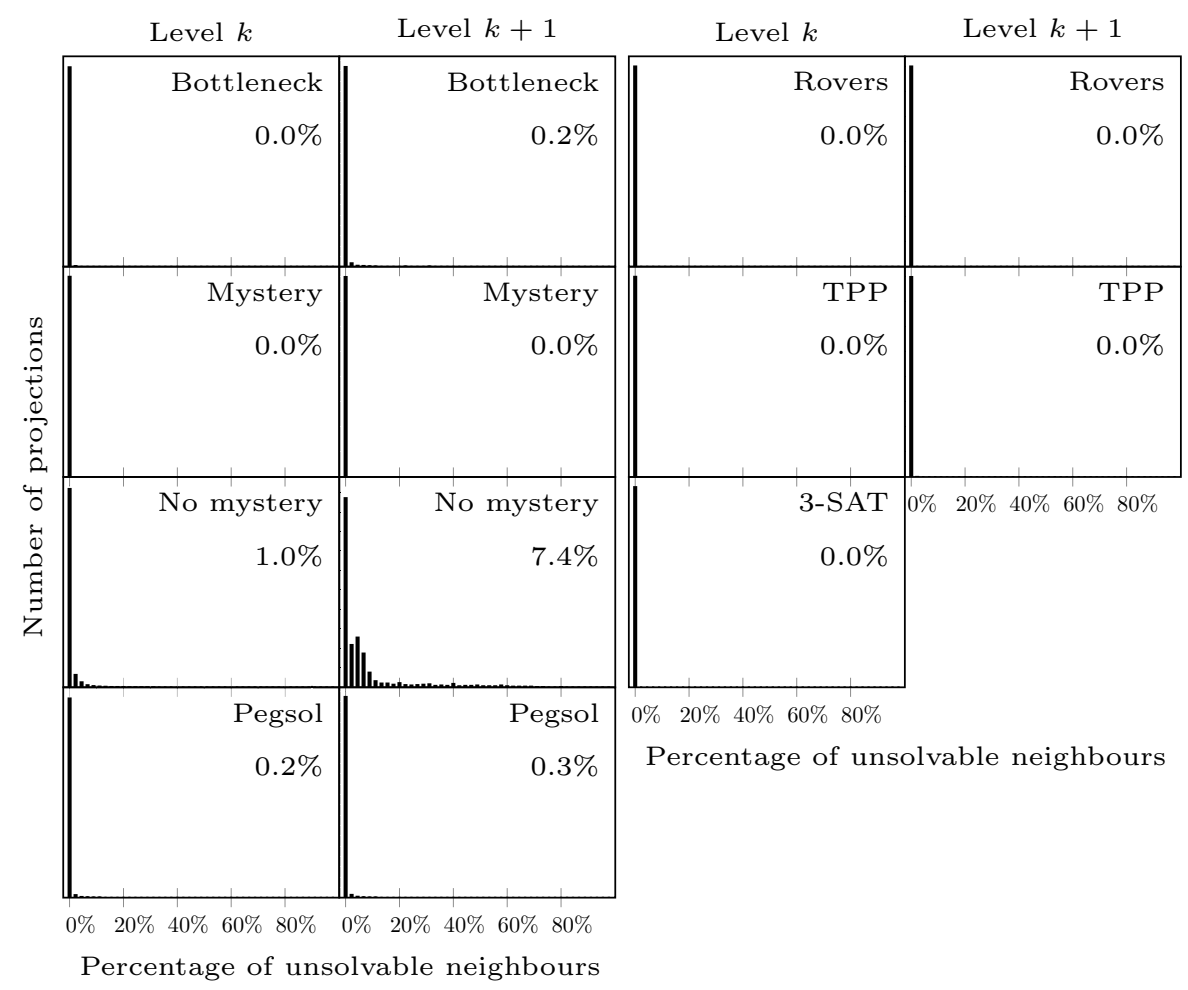

Figure 5.3: Histograms of the ratio between number of unsolvable neighbours and the total number of neighbours that all projections have. The histograms to the left are for the lowest inconsistent level $k$, and the corresponding histograms to the right are for the next level. 
if the neighbourhoods are similar and can be bridged by a common unsolvable projection in the next level.

Considering that most domains have very few neighbourhoods, another natural question is if there are variables that always occur together or not at all in every unsolvable projection? We say that such variables at some level are inseparable. The average number of inseperable pairs and triples is given in Table 5.3. For domains with very small neighbourhoods, the number of pairs and triples tends to be higher. One explanation is that projections from different neighbourhoods are likely to not have many variables in common, and since there are only few projections from the same neighbourhood, then the pair and triples are likely to be inseparable. In general, it seems that as the average neighbourhood size increases, the number of inseparable variable subsets decreases. If we could identify inseparable pairs and triples then we could reduce the number of projections to check significantly, e.g. to decide if an instance $\Pi$ is $k$-variable consistent we would normally have to check $\left(\begin{array}{c}|V(\Pi)| \\ k\end{array}\right)$ projections, but if we know of an inseparable pair then we only have to check $\left(\begin{array}{c}|V(\Pi)| \\ k-1\end{array}\right)$ projections since we can treat the pair as a single 'variable'.

\subsubsection{Predecessor and Successor Projections}

Table 5.1 showed that the ratio between the number of unsolvable projections and the total number of projections tends to increase from one level to the next, and we take a closer look at this through the lens of predecessor, successor and minimal projections.

Definition 5.6. The projection $\left.\Pi\right|_{V_{1}}$ is a predecessor to the projection $\left.\Pi\right|_{V_{2}}$ if $\left|V_{1}\right|=\left|V_{2}\right|-1$ and $V_{1} \subset V_{2}$. Conversely, $\left.\Pi\right|_{V_{2}}$ is a successor of $\left.\Pi\right|_{V_{1}}$. Furthermore, we require the causal graph of the instances to be weakly connected and the goal to be defined for at least one variable.

For the lowest inconsistent level $k$, every predecessor is solvable. Hence, the notion is only relevant for levels higher than $k$. Furthermore, an unsolvable projection is minimal if it has no unsolvable predecessors, e.g. all unsolvable projections at the lowest inconsistent level $k$ are minimal. An unsolvable projection at level $>k$ can be minimal, but if it is not then it can be projected into one.

How many unsolvable predecessors that unsolvable projections tend to have is detailed in Figure 5.4, and on average they have about 27\% unsolvable predecessors. The ratio for most domains is around that 


\begin{tabular}{lrrrrr}
\hline & \multicolumn{2}{c}{ \# of neighbourhoods } & & \multicolumn{2}{c}{ Size of neighbourhoods } \\
\cline { 2 - 3 } Domain & Level $k$ & Level $k+1$ & & Level $k$ & Level $k+1$ \\
\hline 3-SAT & 1 & - & 213.8 & - \\
Bottleneck & 2.35 & 1 & 33.53 & 2849.25 \\
Mystery & 1 & 1 & 1.25 & 36.25 \\
No Mystery & 1.12 & 1 & 17.5 & 223.9 \\
Pegsol & 1474.95 & 1628 & 21.6 & 21.67 \\
Rovers & 2.33 & 1 & 1 & 294 \\
TPP & 1 & 1.5 & 1 & 30.5 \\
\hline
\end{tabular}

Table 5.2: The average number of neighbourhoods and their average size for every domain, at the lowest inconsistent level $k$ and at level $k+1$. The average number of unsolvable projections per instance is the product of the number of neighbourhoods and their size.

\begin{tabular}{lrrrrr}
\hline & \multicolumn{2}{c}{ \# of inseparable pairs } & & \# of inseparable triples \\
\cline { 2 - 3 } \cline { 5 - 6 } Domain & Level $k$ & Level $k+1$ & & Level $k$ & Level $k+1$ \\
\hline 3-SAT & 0 & - & 0 & - \\
Bottleneck & 0.95 & 0.85 & 0.6 & 0.6 \\
Mystery & 5.5 & 5.5 & 5.25 & 5.25 \\
No Mystery & 2.36 & 1 & 1 & 0 \\
Pegsol & 2.19 & 0 & 2 & 0 \\
Rovers & 6 & 4.34 & 2.67 & 2.67 \\
TPP & 7.4 & 3.5 & 7.2 & 1.5 \\
\hline
\end{tabular}

Table 5.3: The average number of inseparable pairs and triples for every domain, at the lowest inconsistent level $k$ and at level $k+1$. 
mark, and for Mystery it is a lot higher: $55.8 \%$. The histograms are quite narrow for most domains, except for Mystery and No Mystery, which have quite a wide histogram. Pegsol is, again, the outlier and it is not surprising considering how little the average size of neighbourhoods increased from level $k$ to level $k+1$ : since every successor of an unsolvable projection is also unsolvable and the size of the neighbourhoods did not increase, then there must be very few successors.

The number of unsolvable projections at level $k+1$ that are also minimal is given in Table 5.4, and for most domains there are very few minimal unsolvable projections at level $k+1$, i.e. nearly all unsolvable projections appear to have propogated from some unsolvable predecessor. Yet again, Pegsol is the black sheep, who almost exclusively had minimal unsolvable projections, although, the previous observation explains this too (there must be very few unsolvable successors that are not filtered away).

The main reason (excluding Pegsol, of course) for why the ratio between unsolvable and solvable projections increased is because unsolvable projections give rise to unsolvable successors, i.e. they propagate to the next level (and so on). Furthermore, successors of the same unsolvable projection are also neighbours. This means that, for most domains, we can expect the density of unsolvable projections and neighbours to increase strictly.

\subsection{Experimental Analysis}

A common way of evaluating methods on a large collection of benchmarks is to give the methods a time and space limit, and then count how many they were able to solve within these limits. This is a bit different from the original publication [BJS13], where we also measured their runtime. The reason for this adjustment is that there were no benchmarks of unsolvable instances, so these had to be constructed from scratch. Hence, we only had 11 benchmarks and were able to do a more detailed analysis per instance. However, at this point in time there are many unsolvable instances available and we opted to use them instead in the forthcoming analysis. As the reader will notice, we do not use every domain in the analysis, and we restrict the competition to what was available then. We do this to demonstrate what the original publication [BJS13] demonstrated. 


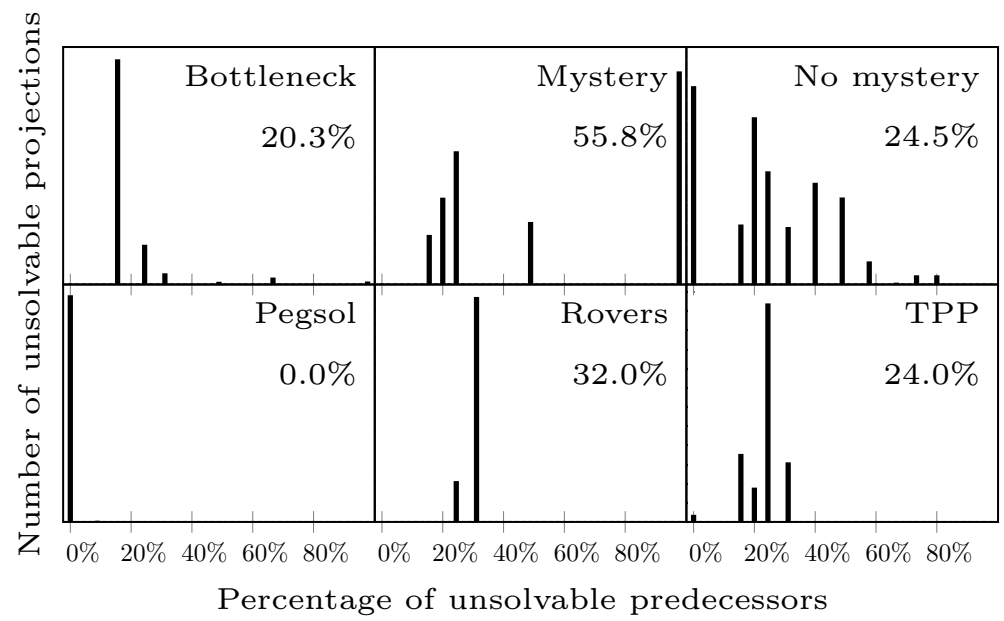

Figure 5.4: Histograms for every domain over how many (in percent) unsolvable predecessors every unsolvable projection has, for one level past the lowest inconsistent level.

\begin{tabular}{lr}
\hline$\%$ of unsolvable projections that are minimal at level & $k+1$ \\
\hline Bottleneck & 0.0 \\
Mystery & 0.0 \\
No Mystery & 31.9 \\
Pegsol & 98.8 \\
Rovers & 0.0 \\
TPP & 1.5 \\
\hline
\end{tabular}

Table 5.4: The percentage of unsolvable projections that are also minimal at lowest inconsistent level $k+1$. 


\subsubsection{Planners}

Consistency checking (CC) used CPT to check projections, and CPT was configured to use $\mathrm{A}^{*}$ coupled with a pattern collection, which consisted of every pattern of size $3 .^{1}$ (The terminology of pattern databases is presented in Section 6.1.) Furthermore, since we are only interested in Plan Existence, $\mathrm{A}^{*}$ did not reprioritise states nor construct a plan. CC ran with both filters until it either found an unsolvable projection or ran out resources. Note that, we do not exploit the observations in Section 5.3 since they were not part of the original method, but we will revisit this topic in Chapter 8, which presents some additional improvements.

We compare consistency checking with a number of standard planners that are optimised for Plan Generation:

- Fast Forward (FF) [HN01]. The planner was set to its standard configuration.

- Fast Downward (FD) [Hel06a]. This planner was tested in two configurations, seq-sat-fdss- 1 and seq-sat-fdss-2, referred to as FD1 and FD2, respectively.

- LAma [RW10]. This is yet another configuration of FD, seq-satlama-2011.

It is reasonable to assume that the heuristics of these planners will often do more harm than good on unsolvable instances. There are some methods that are primarily intended for planning that are reasonable to use also for detecting unsolvable instances. We also evaluated $\mathrm{A}^{*}$ coupled with the following admissible heuristic functions: (i.e. Cost Optimal Plan Generation)

- Blind. This heuristic function returns 1 if the state is not a goal state, 0 otherwise. The blind heuristic will always be better than any heuristic function that cannot detect dead-ends since it does not waste any resources.

- iPDB $\left[\mathrm{HBH}^{+} 07\right]$. There are many approaches of constructing pattern collections, iPDB is one well-known way of doing so and is discussed in more detail in Section 6.1. We used the default settings in FD, i.e. the maximum PDB size is 2 million and the maximum collection size is 20 million.

\footnotetext{
${ }^{1}$ The original paper used another configuration.
} 
- $h^{m}$ [HG00]. This is a family of admissible heuristic functions. For a given $m \geq 1, h^{m}$ is the maximum cost of achieving any subset of $m$ goal atoms by regression, applying the same criterion also to all subgoals. CPT was configured to use depth-first search, which used $h^{m}$ for dead-end detection. If this state is identified as a dead-end, then the planner can backtrack early.

\subsubsection{Coverage}

All tests were run a computer with an Intel Xeon E5-2660 at $2.2 \mathrm{GHz}$ CPU and 4 GB available RAM. All methods were given a time limit of 30 minutes on each instance, and the empirical data for all methods is listed in Table 5.5.

The worst performing contender was blind search, which was able to prove a mere 18 instances as unsolvable by exhaustively enumerating every reachable state and check if none is a goal state. In general, we cannot expect this to work since there often are too many states to store in memory, thus this method serves as a baseline.

The planner configurations FF, FD1, FD2 and LAMA outperformed blind search and were able to prove 19, 32, 34 and 23 instances as unsolvable, respectively. It is worth noting that some of these configurations consist of several searches, and each search might use a different heuristic function. Regardless, they did not provide any meaningful improvement over blind search and were easily outperformed by the admissible heuristics iPDB, $h^{2}$ and $h^{3}$, which were able to prove 48,39 , and 55 instances as unsolvable. Overall, no method except for $h^{3}$ could compete with $\mathrm{CC}$ and both identified a significant number of initial states as dead-ends (CC proves that an initial state is a dead-end by finding an unsolvable projection, and the heuristic function $h^{3}$ proves this by producing the value $\infty$ ). However, $h^{3}$ is very expensive to compute and for most instances, just evaluating the initial state can take up to half a minute (if not more).

The best performing method was $\mathrm{CC}$, which proved a total of 66 instances as unsolvable. The most surprising observation was that iPDB identified 17 TPP instances as unsolvable, but CC only identified 4, despite that both methods are based on variable projection. However, they identified the same initial states as dead-ends, i.e. iPDB made use of good dead-end detection to prune the reachable state transition graph. 


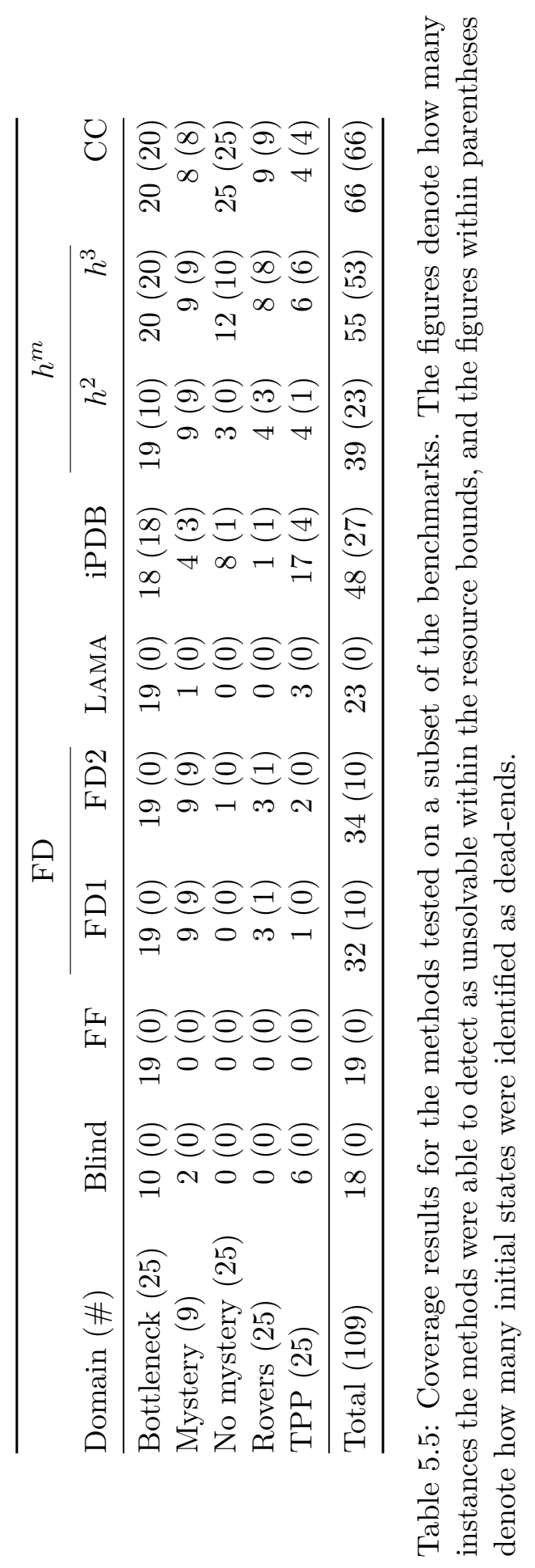




\subsubsection{Discussion}

The experiments were meant to illustrate a shortcoming of planners tailored toward solvable instances. However, we have also conveniently ignored some domains that would highlight a weakness of $\mathrm{CC}$, which we will discuss shortly. The configurations of the planners FF, FD and LAMA, were optimised for Plan Generation (PG), and one would assume such planners to be good at the corresponding problem Plan Existence (PE), however, this was obviously not the case since they just marginally outperformed blind search. The planner configurations for Cost Optimal Plan Generation (COPG), iPDB and $h^{m}$, were more successful, despite tackling a tougher problem.

Consider the following two properties of heuristic functions, where $h$ is some heuristic function and $h^{*}$ is the perfect heuristic function.

1. Let $s$ be a state. If $h(s)=\infty$ then $h^{*}(s)=\infty$.

2. For every state $s, h(s) \leq h^{*}(s)$.

A heuristic function is safe if it fulfills property (1), and it is admissible if it also fulfills (2). The heuristic functions of the planners were safe, and heuristic functions for PG need only to fulfill (1). However, it appears that admissible heuristic functions for COPG put more emphasis on (1) than non-admissible heuristic functions, despite (1) obviously being useful for both problems - a perplexing anomaly. After all, (2) is not important for unsolvable instances, thus there is no reason why safe non-admissible heuristic functions should perform worse than admissible ones.

There is a strong correlation between the instances that $\mathrm{CC}$ were able to prove unsolvable and the data in Table 5.1. The lowest inconsistent level for the domains that CC performed well on is quite low, but it is also low for the few instances of Rovers and TPP that the table had data on. Recall that we used $a$ lot more time to collect the data in Table 5.1, however, that did not help very much for Rovers and TPP. This exposes a weakness of CC, namely, that if there is no small unsolvable projection then spending more time is likely to be fruitless. This is especially clear in TPP, where iPDB was able to prove 17 instances as unsolvable and CC only 4. Note that the data in Table 5.1 in incomplete and it is likely that for the remaining instances, the lowest inconsistent level is quite high. In Chapter 6 we will take a look at how to remedy this by taking advantage of dead-ends identified whilst solving projections, using a more aggressive strategy, and by making projections more informative with the help of mutexes. 
A much more extensive experimental analysis is presented in Section 6.5, where we use more benchmarks and also compare against methods developed since the original paper was published. 


\section{Chapter Six Pattern Databases}

A major drawback of consistency checking is that, when it does not find an unsolvable projection, then all the work is wasted since it does not save any information about the solved projections. However, there is useful information to store, namely, dead-end states. We remind the reader that no goal state is reachable from a dead-end state. A dead-end state of a projection can match several states of the original instance, and all of them are dead-ends. Furthermore, all states reachable from a dead-end are also dead-ends. Consequently, dead-end detection can have a significant impact on the performance of a planner. After all, the initial state of an unsolvable instance is a dead-end, and the planner is done if it can detect it as such.

A natural continuation of consistency checking is thus to solve projections completely with regression search, and store all states that never got visited since they are dead-ends. If the initial state of a projection was never visited then we have proven that the original instance is unsolvable. And when we have spent ample time on solving projections, then we turn our focus on the original instance and perform a search that ignore states that match any stored dead-end state. This is one of the differences between the methods devised in this chapter and consistency checking. A pitfall of consistency checking is how reluctant it is to check larger projections, and the second difference is that the devised methods are a lot more eager to check larger projections.

In Section 6.1 we give a brief introduction to pattern databases [CS98] and present a strategy that we use to build them. We present the methods that we analyse in Section 6.2 and carry out a detailed statistical analysis of them in Section 6.3, which is similar to the one we did for consistency checking. For this statistical analysis, we took advantage of a large computer cluster to determine a distribution for every instance of how many dead-ends the projections have. These distributions are 
then used in Section 6.3.1 to show that the proposed methods construct pattern collections that are amongst the best. In other words, to improve dead-end detection further would require additional techniques. We show in Section 6.3.2 that the two proposed methods differ in a significant way: one method tends to find more unsolvable projections than the other, which is important since an unsolvable projection implies that the instance is unsolvable. In Section 6.4, we show that mutexes are useful for improving how many dead-ends patterns can detect. Finally, in Section 6.5 we run experiments and show that our best methods are competitive. The experiments also show that mutexes have a considerable effect on many instances.

\subsection{Definitions}

We first give a brief introduction to pattern databases, and present one technique for constructing pattern collections. A pattern $P \subseteq V(\Pi)$ is a subset of variables of an instance $\Pi$, and the lowest solution cost of reaching a goal state in $\left.\Pi\right|_{P}$ from each state $s \in$ TotalStates $\left(\left.\Pi\right|_{P}\right)$ is stored in a pattern database (PDB) $h^{P}$. The database is used to efficiently provide an estimate of the real solution cost from some state to a goal state. We write $h^{P}(s)$ to denote the cost associated with $s$ in $h^{P}$. A main problem with constructing a PDB is how to choose the pattern, since PDBs are often vastly different with regard to how informative they are (and there is an enormous amount of them). A pattern collection $C$ consists of several patterns which, together, are used to provide a solution cost estimate: $C(s)=\max \left\{h^{P}(s): P \in C\right\}$. We are only interested in dead-end detection and for this purpose it is sufficient to take the maximum, however, for an actual solution cost estimate it might be beneficial to take the sum of a few, carefully selected, PDBs.

A popular strategy to build a pattern collection is $i P D B\left[\mathrm{HBH}^{+} 07\right]$. The iPDB strategy uses hill-climbing search in the space of pattern collections, and we use a similar strategy. Hill-climbing search is an optimisation technique which attempts to maximise some score function by local search. In planning, the score function for solvable instances is often the average cost in the PDB. The search starts with a pattern of a single goal variable, and then attempts to find a better pattern by repeatedly adding a single variable to it. There might be several candidates, and the candidate whose PDB scored best is selected. This procedure is repeated until some condition is met. More precisely, for 
an instance $\Pi$, we use the following pattern selection strategy.

1. Let $P=\{g\}$ where $(g, d) \in G(\Pi), d \in D_{g}$ (i.e. $g$ is a goal variable), and let $b$ be a bound on the maximum PDB size. We also have some function Score, where the input is a PDB and the output is a number;

2. Compute $\operatorname{Score}\left(h^{P \cup\{v\}}\right)$ for every $v \in V(\Pi)$, where $v \notin P, v$ is weakly connected to some variable in $P$ in the causal graph, and $\left|D_{v}\right| \cdot \prod\left\{\left|D_{v^{\prime}}\right|: v^{\prime} \in P\right\} \leq b$.

3. If there are no candidates then return $P$; and

4. Let $P$ be the candidate with the highest score in step 2 and go to step 2. If there are several candidates with the same score then choose one arbitrarily.

The resulting pattern is added to the pattern collection, and then we start yet another search (with another initial pattern, if there is one). This is repeated until either: the collection is sufficiently large; or if the last 5 searches returned patterns that we have already seen before. The implementation tries to avoid candidates that were selected in previous searches. For example, suppose there are two candidates $C_{1}$ and $C_{2}$, $\operatorname{Score}\left(C_{1}\right)>\operatorname{Score}\left(C_{2}\right)$. If $C_{1}$ was selected in a previous search but not $C_{2}$, then $C_{2}$ is selected. And if both were selected in previous searches, then $C_{1}$ is selected. The purpose of this is to avoid selecting the same pattern several times when constructing a pattern collection.

\subsection{Methods}

We propose two methods for constructing pattern collections and both methods are based on hill-climbing search, and the only difference is the score function. Before we define them, we give a definition of a central concept. Let $\Pi$ be a planning instance. The function $\xi(\Pi)$ generates a set of at most 20000 reachable states of $\Pi$ by breadth-first search. Note that, if $\Pi$ is unsolvable then every state of $\xi(\Pi)$ is a dead-end. The function $\xi$ is required to generate the same set every time for the same instance. The figure 20000 is somewhat arbitrary, and we discuss why it is sufficiently large later on. Our methods are: 
- $H C_{D E}(\Pi)$ : A pattern collection is constructed for $\Pi$ by hillclimbing search with the scoring function:

$$
D E\left(h^{P}\right)=\frac{\mid\left\{s \in \text { TotalStates }\left(\left.\Pi\right|_{P}\right): h^{P}(s)=\infty\right\} \mid}{\mid \text { TotalStates }\left(\left.\Pi\right|_{P}\right) \mid}
$$

which favours PDBs with a high percentage of dead-ends.

- $H C_{\text {Samples }}(\Pi)$ : A pattern collection is constructed for $\Pi$ by hillclimbing search with a scoring function that attempts to maximise the number of different dead-end states that the pattern collection can detect. If two patterns are able to identify exactly the same states as dead-ends, then we only need one of them. The overlap of a new pattern with the collection is approximated with the help of $\xi(\Pi)$ in the following way. Let $P$ be a pattern and let $C$ be the current collection, then the scoring function is:

$$
\operatorname{Samples}\left(h^{P}, C\right)=\left|\left\{s \in \xi(\Pi): h^{P}(s)=\infty, C(s) \neq \infty\right\}\right|
$$

The function Samples outputs the number of dead-ends that $C$ did not detect as dead-ends, but $h^{P}$ did detect. Note that, $\xi$ generate states close to the initial state. Detecting dead-end states close to the initial state is likely to prune away many reachable states from the search space. Hence, Samples should favour patterns that can detect many, previously undetected, dead-end states of $\xi(\Pi)$. Furthermore, we resolve tiebreaks with $D E\left(h^{P}\right)$ (and if we still have a tiebreak then the successor is chosen arbitrarily).

\subsection{Statistical Analysis}

We present a detailed statistical analysis of how well pattern collections constructed by the methods defined in Section 6.2 perform on benchmarks. Roughly speaking, the analysis reveals the limit of pattern collections as a method for detecting dead-ends, and we attain this by letting a computer cluster ${ }^{1}$ generate PDBs using many years of CPUtime. The collected data lets us visualise the distribution of PDBs w.r.t. how many dead-ends they have - a crucial property for good dead-end detection. We show that the methods perform comparably but that $H C_{D E}$ tends to find more unsolvable projections, which is important since such projections prove that the instance is unsolvable.

\footnotetext{
${ }^{1}$ The processors were 8 -core Intel Xeon E5-2660 at $2.2 \mathrm{GHz}$, provided by National Supercomputer Centre (NSC) at Linköping University. Website: https://www.nsc.liu.se/
} 


\subsubsection{Dead-end Detection}

A disadvantage of a typical experimental analysis, such as measuring coverage, is the absence of a notion of optimality. For example, let $h^{P}$ be a PDB whose size is bounded by some integer $b$, and let $s$ be a dead-end state that $h^{P}$ cannot detect. Then we do not know if there is another PDB, whose size is also bounded by $b$, that can detect $s$. An optimal pattern collection for the bound $b$ would contain a PDB for every dead-end that is detectable by a PDB whose size is bounded by $b$. Note that there might be dead-ends that an optimal pattern collection does not detect, for example, the initial state if there is no PDB of an unsolvable projection whose size is bounded by $b$. In this section, we analyse how well the pattern collections constructed by $H C_{D E}$ and $H C_{\text {Samples }}$ are at detecting dead-ends, and whether spending more CPU-time would help. The latter is estimated by comparing how many dead-ends they detect compared to pattern collections that were constructed by using years of CPU-time on a computer cluster. The dead-ends that we use to compare them against are generated by $\xi$.

It is, of course, computationally infeasible to construct optimal pattern collections in general, and a more pragmatic approach is to spend an excessive amount of CPU-time instead. We used a computer cluster to generate 20 million random PDBs for every instance $\Pi$ where the size of every PDB is bounded by 500000 , and we denote this set of PDBs as $\mathscr{P}(\Pi)$. The pattern collection $\varphi(\Pi) \subseteq \mathscr{P}(\Pi)$ consists of the 1500 patterns with the highest percentage of dead-ends. We stress that even though we generate 20 million PDBs, it is not a complete set of PDBs for most instances, and it is possible for $H C_{D E}$ and $H C_{\text {Samples }}$ to generate PDBs not in $\mathscr{P}(\Pi)$. The reason why we do not generate more or larger PDBs is because of how computationally expensive it is. Of course, the same bound on PDB size was used by $H C_{D E}(\Pi)$ and $H C_{\text {Samples }}(\Pi)$ in the following analysis.

Figure 6.1 and 6.2 shows how $H C_{D E}$ and $H C_{\text {Samples }}$ perform against $\varphi$, where hill-climbing methods were given at most 20 minutes to construct a pattern collection. The pattern collections perform similarly and in most domains they were able to detect as many dead-ends as $\varphi$. The exceptions are Pegsol and 3-SAT, which we examine more closely later. In the other domains the ratio is close to 1, i.e. $H C_{D E}$ and $H C_{\text {Samples }}$ detected about as many dead-ends as $\varphi$. We observe the effect of $\mathscr{P}(\Pi)$ being incomplete in 3-SAT, where $H C_{D E}$ and $H C_{\text {Samples }}$ detect many more dead-ends than $\varphi$. Typically, the frequency of deadends drop as the instance difficulty increase (roughly speaking, the 


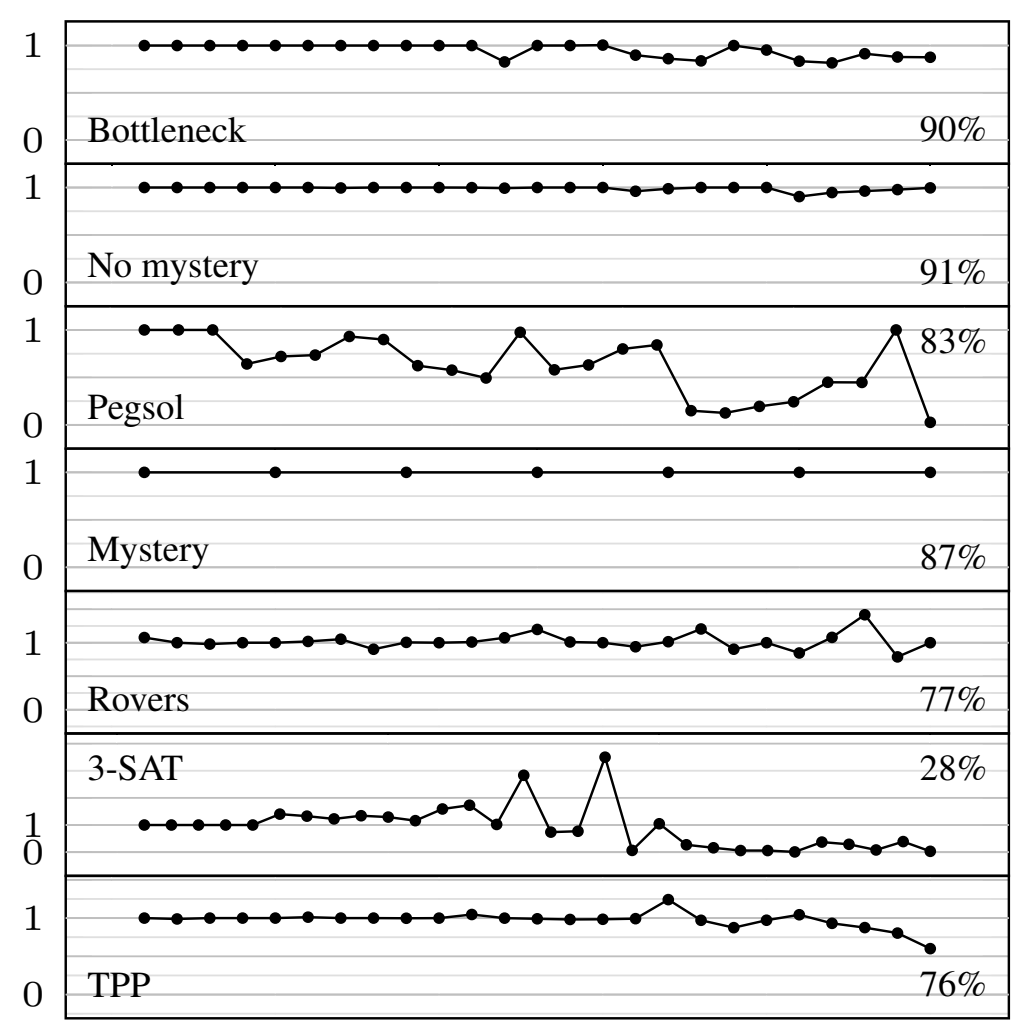

Figure 6.1: Every point represents an instance $\Pi$ and they are ordered according to increasing difficulty (left to right). The value of a point is the ratio of the number of dead-ends identified by $H C_{D E}(\Pi)$ to the number of dead-ends detected by $\varphi(\Pi)$ on the set $\xi(\Pi)$. A point below 1 means that the pattern collection identified fewer dead-ends than $\varphi(\Pi)$, and the percentage is the average ratio of dead-ends that $\varphi(\Pi)$ detected of $\xi(\Pi)$. No PDB detected any dead-end for Tiles. 


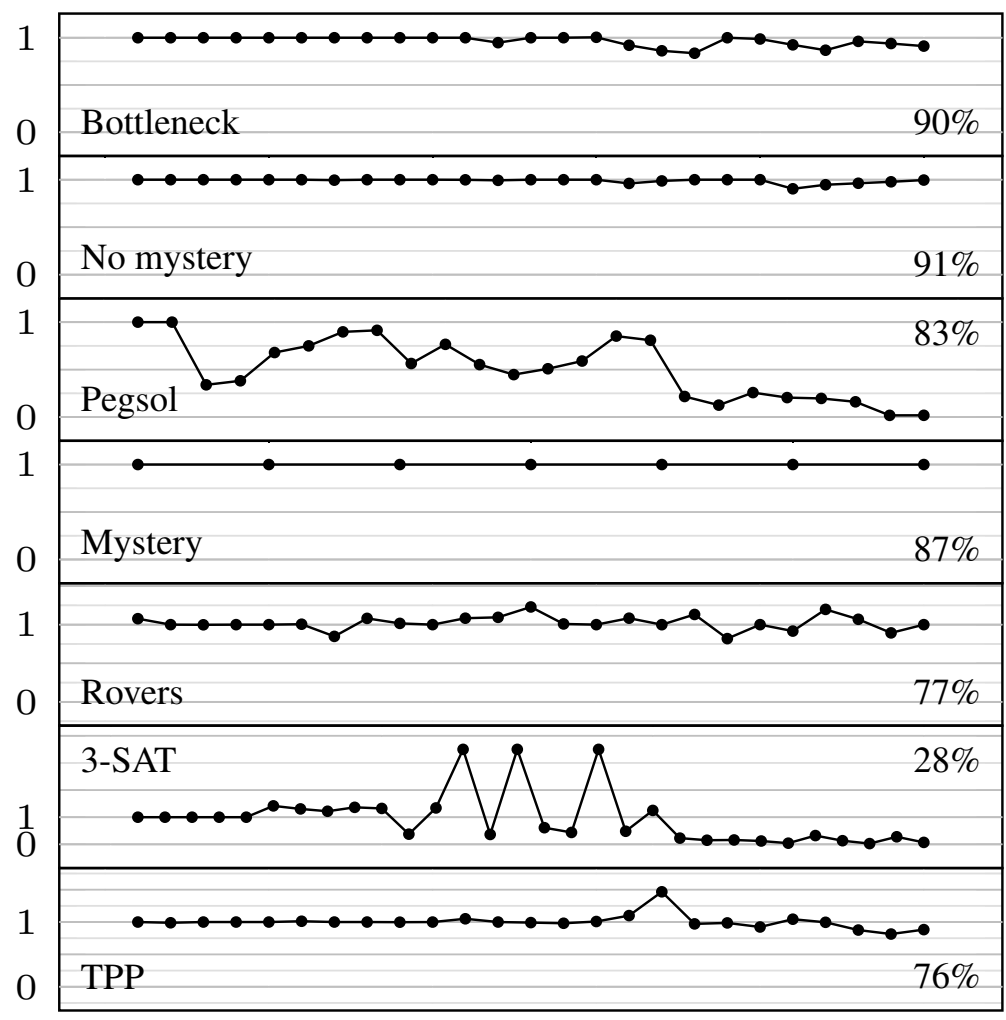

Figure 6.2: Same as Figure 6.1 except that we used $H C_{\text {Samples }}$. 


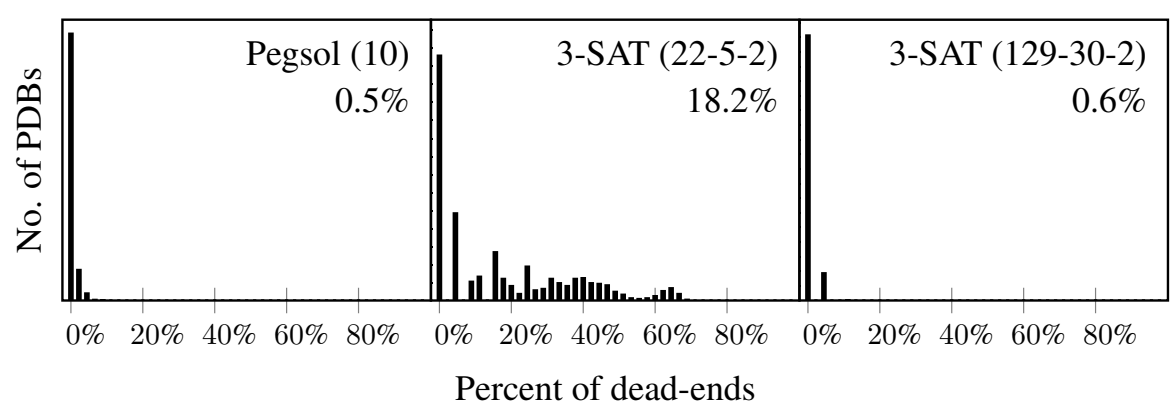

Figure 6.3: Histograms of the percentage of dead-ends of the PDBs in $\mathscr{P}$ for 3 instances. The numbers within the parentheses denote specific instances in the benchmark. The percentage in the upper right corner is the average percent of dead-ends across all PDBs of the instance.

difficulty of an instance is its state space size, or by the parameters used to generate it), i.e. good patterns become more rare. Consider this: $\varphi$ was given several weeks of CPU-time per instance to generate 20 million PDBs and construct a pattern collection, whereas $H C_{D E}$ and $H C_{\text {Samples }}$ were given a mere 20 minutes and they were still able to compete with $\varphi$ !

The two outliers are Pegsol and 3-SAT, but why is this? Figure 6.3 shows the histograms of how many dead-ends the PDBs of $\mathscr{P}$ have for 3 instances: a hard Pegsol instance, an easy 3-SAT instance and a hard 3-SAT instance. The easier 3-SAT instance has a smaller state space than the harder one which means that the PDBs for the easy instance are (relatively) a lot more informative than the PDBs for the hard instance. To get a more meaningful distribution for the hard instance we might have to consider larger PDBs or additional techniques (e.g. mutexes). The situation is different for Pegsol, where the distribution in Figure 6.3 is representative for every Pegsol instance, i.e. the PDBs are not very informative. The distributions are even worse for smaller PDBs, i.e. no $\mathrm{PDB}$ has any dead-end. The methods $H C_{D E}$ and $H C_{\text {Samples }}$ might struggle when given these instances because their score functions count dead-ends for smaller state space sizes but the score is almost always 0 . In other words, the search degrades to blind, uninformed search and is therefore unlikely to find the few patterns which are useful for dead-end detection. 


\subsubsection{Unsolvable Projections}

If we encounter an unsolvable projection at some point during the construction of a pattern collection then we can terminate early: we know that the initial state is a dead-end and that the instance is unsolvable. Hence, unsolvable projections are very important. The proposed methods differ in this regard; $H C_{D E}$ found a total of 1458 unsolvable projections over all instances, whereas $H C_{\text {Samples }}$ only found 499. To derive these figures, we did not terminate early, and every pattern collection contained at most $60 \mathrm{PDBs}$ of unsolvable projections. However, this does not mean that $H C_{D E}$ finds a single unsolvable projections faster, or more likely, than $H C_{\text {Samples }}$. In this section, we investigate why $H C_{D E}$ finds more unsolvable projections than $H C_{\text {Samples }}$, and whether the PDBs of unsolvable projections tend to have a lot of dead-ends in addition to the initial state, i.e. if such PDBs are likely to get a high score by $D E$.

\section{- Note}

We give a short example to illustrate how the figures in Table 6.1 are computed. Suppose we have three instances $\Pi_{1}, \Pi_{2}$ and $\Pi_{3}$ from the same domain $\mathcal{D}$. For each instance $\Pi_{i}, 1 \leq i \leq 3$ we compute 100 PDBs, denoted $h_{i}^{P_{1}}, \ldots, h_{i}^{P_{100}}$. Then $H_{i}$ is the histogram over the ratios $D E\left(h_{i}^{P_{1}}\right), \ldots, D E\left(h_{i}^{P_{1} 00}\right)$.

Suppose $\left.\Pi_{i}\right|_{P_{1}},\left.\Pi_{i}\right|_{P_{2}}$ and $\left.\Pi_{i}\right|_{P_{3}}$ are unsolvable, and are the only unsolvable projections. Let $r_{i}^{1}, r_{i}^{2}$ and $r_{i}^{3}$ be the percentile ranks of the PDBs of these unsolvable projections in $H_{i}$. The percentile rank of a $\mathrm{PDB}$ is its relative position in a distribution, e.g. if the percentile rank of a PDB is 99 then $99 \%$ of the PDBs have a smaller or equal ratio of dead-ends. The figures in Table 6.1 are computed in the following way. Let $r_{i}=\operatorname{avg}\left\{r_{i}^{1}, r_{i}^{2}, r_{i}^{3}\right\}$. Then: $\min \left\{r_{i}: 1 \leq i \leq 3\right\}$ is the minimum-average; $\operatorname{avg}\left\{r_{i}: 1 \leq i \leq 3\right\}$ is the average-average; and $\max \left\{r_{i}: 1 \leq i \leq 3\right\}$ is the maximumaverage. These figures are only for $\mathcal{D}$.

Table 6.1 shows that PDBs of unsolvable projections tend to have a high percentile rank in the distribution of frequency of dead-ends, i.e. PDBs of unsolvable projections tend to have many dead-ends. For example, Figure 6.3 illustrates this distribution for 3 instances. Note that a high percentile rank of a PDB for the Pegsol instance means that the PDB has around $10 \%$ dead-ends (i.e. a high percentile rank does not guarantee good dead-end detection but that it is amongst the best 


\begin{tabular}{lrrr}
\hline & \multicolumn{3}{c}{ Average percentile rank } \\
\cline { 2 - 4 } Domain & Minimum & Average & Maximum \\
\hline 3-SAT & 98 & 98 & 99 \\
Bottleneck & 49 & 67 & 93 \\
Mystery & 51 & 75 & 99 \\
No mystery & 96 & 97 & 99 \\
Pegsol & 96 & 98 & 99 \\
Rovers & 99 & 99 & 99 \\
TPP & 99 & 99 & 99 \\
\hline
\end{tabular}

Table 6.1: Minimum, average and the maximum average percentile rank of the ratio of dead-ends in the PDBs of the unsolvable projections in $\mathscr{P}(\Pi)$, for every instance $\Pi$. That is, for every instance of a domain we take the average percentile rank of the unsolvable projections, and then we take the minimum, the average or the maximum (of the aforementioned average) over the entire domain.

for said purpose). For most domains, the percentile ranks are very high, around 98, which means that PDBs of unsolvable projections tend to score high by $D E$. This partly explains why $H C_{D E}$ encounters far more unsolvable projections than $H C_{\text {Samples: }}$ : $D E$ serves as a heuristic function to find unsolvable projections. The two exceptions are Bottleneck and Mystery, but their average percentile rank is higher than 50, i.e. they have more dead-ends than the average PDB.

\subsection{Mutual Exclusions}

A useful and well-known technique to improve the heuristic values of a PDB is to use mutexes: a mutex is a partial state which matches states that cannot be reached from the initial state. We generate mutexes with the critical path heuristic $h^{m}$ [HG00]. More specifically, we let $m=2$ or $m=3$. In this section, we detail how many mutexes $h^{2}$ and $h^{3}$ identify and whether any of the mutexes can directly prove the instance unsolvable. If a mutex does not infer unsolvability, then it can still have a tremendous effect on increasing the number of dead-ends in a PDB.

Definition 6.1. A partial state $s_{m}$ of a $\mathrm{SAS}^{+}$instance $\Pi$ is a mutual exclusion, or mutex, if and only if there is no state $s$ reachable from $I(\Pi)$ such that $s_{m} \subseteq s$. 


\begin{tabular}{|c|c|c|c|c|}
\hline \multirow[b]{2}{*}{ Domain (\# instances) } & \multicolumn{2}{|c|}{ Mutexes identified } & \multicolumn{2}{|c|}{ Goal is mutex } \\
\hline & $h^{2}$ & $h^{3}$ & $\overline{h^{2}}$ & $h^{3}$ \\
\hline 3-SAT $(30)$ & 42 & 38554 & 0 & 0 \\
\hline Bottleneck (25) & 51679 & 248340 & 10 & 20 \\
\hline Mystery (9) & - & - & 9 & 9 \\
\hline No mystery (25) & 81788 & 2027164 & 0 & 10 \\
\hline Pegsol (24) & 826 & 4768 & 0 & 6 \\
\hline Rovers (25) & 19431 & 234424 & 3 & 8 \\
\hline Tiles (20) & 11160 & 11160 & 0 & 0 \\
\hline $\operatorname{TPP}(25)$ & 97858 & 1479852 & 1 & 5 \\
\hline
\end{tabular}

Table 6.2: The sum of mutexes identified for every domain. Mutexes are identified by either $h^{2}$ or $h^{3}$. The sum does not include mutexes for instances whose goal matched a mutex (e.g. Mystery).

A common definition of mutex in the literature is that there is no reachable total state which contains more than one variable-value pair of a mutex, but we use a more general definition (for mutexes of size 2 , both definitions are identical). We get mutexes of size 2 and 3 from $h^{2}$ and $h^{3}$, respectively. Ideally, for an unsolvable instance $\Pi$ we want to prove that some $s_{m} \subseteq G(\Pi)$ is a mutex (i.e. every goal state is unreachable), but it is obviously PSPACE-hard to do so. Hence, if we use a polynomial-time algorithm to identify mutexes then the typical case is that the goal does not match any identified mutex.

To (naively) decide whether a partial state $s_{m}$ is a mutex for an instance $\Pi$, we let $s_{m}$ be the goal and evaluate $h^{2}$ on $I(\Pi)$. Table 6.2 details how many mutexes were found by $h^{2}$ and $h^{3}$, and how many instances were identified as unsolvable by a mutex. Every instance of the Mystery domain had a goal which matched a mutex as well as many instances of the Bottleneck domain. Mutexes from $h^{2}$ immediately identified 23 of 183 instances as unsolvable, whilst mutexes from $h^{3}$ identified 58 instances as unsolvable. For every instance, it took less than 4 seconds for $h^{2}$ to identify mutexes, and (except in a few cases) less than 5 minutes for $h^{3}$.

The mutexes identified by $h^{2}$ are also identified by $h^{3}$, but they are in a different form. For example, suppose $h^{2}$ identifies a mutex $s_{m}=$ $\left\{\left(v_{1}, d_{1}\right),\left(v_{2}, d_{2}\right)\right\}$, then $h^{3}$ will generate the mutexes $\left\{s_{m} \cup\left\{\left(v_{3}, d_{3}\right)\right\}\right.$ : $\left.v_{3} \in V \backslash\left\{v_{1}, v_{2}\right\}, d_{3} \in D_{v_{3}}\right\}$. Obviously, this set, collectively, identifies the same states as $s_{m}$. In practice, we first generate mutexes by $h^{2}$ since they are more general, and only add mutexes generated by $h^{3}$ if 


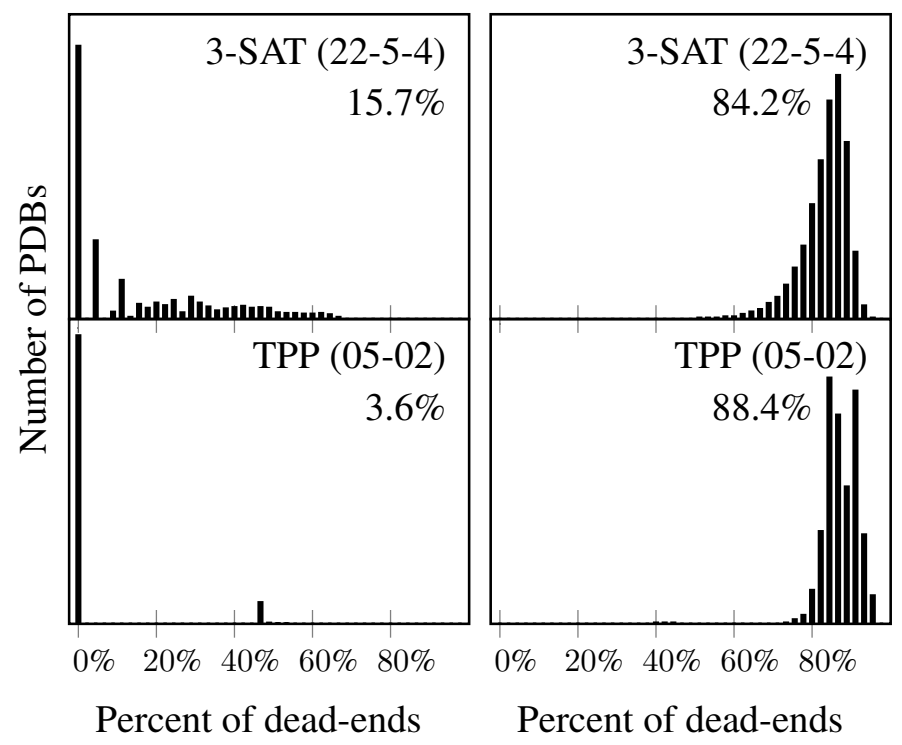

Figure 6.4: Histograms of the percent of dead-ends of the PDBs in $\mathscr{P}$ for two instances. The PDBs in the histograms to the left did not use mutexes, whilst the PDBs in the histograms to the right did. The mutexes were generated by $h^{3}$. No mutex matched any goal.

they are not matched by some more general mutex.

If a solution for a projection visits a state that matches a mutex then there is no corresponding solution for the original instance, and by avoiding such solutions we can generate PDBs with potentially more dead-ends. Figure 6.4 shows the impact of exploiting mutexes when generating PDBs for two instances, and the impact is a very significant increase in dead-ends: the average ratio of dead-ends for the 3-SAT PDBs went from $15.7 \%$ to $84.2 \%$ and for the TPP PDBs it went from $3.6 \%$ to $88.4 \%$. Figure 6.4 consists of only two instances, but the experiments in Section 6.5 show that many other instances benefit greatly, too, from using mutexes.

\subsection{Experimental Evaluation}

In this section, we evaluate our methods on all benchmarks. The tests were run on a computer with an Intel Xeon E5-2660 at $2.2 \mathrm{GHz}$ CPU and 4 GB available RAM, and every method were given 30 minutes for every instance. 


\subsubsection{Planners}

This time we do not compare against configurations meant for solvable instances (i.e. FF, LAMA, and so on), and focuse on methods developed for unsolvable instances. The methods we compare are:

- Blind. Same configuration as we used in Section 5.4.

- Two variants of the M\&S heuristic that are optimised for deadend detection [HKT14]. Hoffmann et al. [HKT14] gave three configurations, and we present the two best ones: Mrg1 and Mrg2.

- Consistency Checking (CC). See Chapter 5 for details. We also evaluate the impact of using mutexes when solving projections, which were generated by $h^{3}$.

- iPDB. This method is not designed for unsolvable instances, but serves as a baseline for our similar methods.

- $H C_{B L}$. A pattern collection is constructed by hill-climbing search with the scoring function:

$$
S\left(h^{P}\right)=\frac{\sum\left\{h^{P}(s): h^{P}(s) \neq \infty, s \in \text { TotalStates }\left(\left.\Pi\right|_{P}\right)\right\}}{\mid \text { TotalStates }\left(\left.\Pi\right|_{P}\right) \mid}
$$

This scoring function is useful for solvable instances, but not so much for unsolvable. This method is very similar to iPDB and the main difference is that this method does not terminate the search if the improvement is too small. The planner used a depth-first search algorithm, and the pattern collection is used to detect dead-ends. Just like iPDB, this method also serves as a baseline.

- $H C_{D E}$. The pattern collection is constructed as previously described, and is using the same planner as $H C_{B L}$. We also evaluate different PDB and pattern collection sizes, and how much PDBs benefit from mutexes generated by $h^{2}$ or $h^{3}$. These parameters are denoted as $C_{m}^{n}$, where $C$ is the pattern collection, $n \geq$ $\max \left\{\mid\right.$ TotalStates $\left.\left(\left.\Pi\right|_{P}\right) \mid: P \in C\right\}, m \geq \sum_{P \in C} \mid$ TotalStates $\left(\left.\Pi\right|_{P}\right) \mid$, and we write $h^{2}$ or $h^{3}$ to denote whether the mutexes were generated by $h^{2}$ or $h^{3}$.

- DE-PDBs [PS16], which identifies dead-ends by systematically constructing PDBs and then use them to prune the reachable state space - very comparable to our method. 
- Aidos $\left[\mathrm{SPS}^{+} 16\right]$, the winner of the first unsolvability IPC, which consists of an array of methods, and one of them is DE-PDBs.

- SymPA [Tor16], which performs bidirectional search in the original and abstract state spaces.

\subsubsection{Coverage}

Coverage results are presented in Table 6.3, together with two different totals. The first total is the sum of coverage for all domains, whilst the second ignores the coverages of 3-SAT, Pegsol and Tiles. We motivate the second total in the following way. Any heuristic function that is evaluated sufficiently fast will perform well on the excluded instances because the blind heuristic function performs well. In other words, we are not measuring how good they are at dealing with the combinatorial explosion. Note that blind search does not have full coverage for the excluded domains, but no other method performed better (except for Aidos in the 3-SAT domain). The domains excluded in second total coverage also coincide with the domains that we did not evaluate in Section 5.4, and a difference between the two totals is especially noticeable for consistency checking (CC): CC was not able to compete with Mrg1 in the first total (77 vs. 114), but could compete in the second total (66 vs. 65). This is because the lowest inconsistent level is too high for $\mathrm{CC}$ even though it is possible to simply visit every reachable state.

The M\&S methods Mrg1 and Mrg2, just like CC, were tailored towards proving that the initial state is a dead-end; in this regard, Mrg1 had the best performance with 114 . The runner ups were $C_{60 M}^{5 M}$ with mutexes by $h^{3}$ and Aidos, who showed 98 initial states as dead-ends (Aidos got coverage if any method detected the initial state as a deadend). When the latter methods had to fall back to search, then their total coverage was higher than Mrg1.

The best performing method w.r.t. total coverage was Aidos which proved 170 (or 106) of 183 (or 109) instances as unsolvable - impressive! However, it is arguably not fair to compare a single method to a collection of methods. Hence, we focus our comparison to the most similar method: DE-PDBs, which is also part of Aidos. Our best performing configuration was $C_{60 M}^{5 M}$ using mutexes generated by $h^{3}$, which identified 147 (or 98) as unsolvable. DE-PDBs identified 132 (or 83 ) instances as unsolvable, which is 15 less than ours. The difference is explained by Mystery and Rovers, where our method had a much 
better coverage thanks to mutexes. When $H C_{D E}$ did not have access to mutexes then its performance was comparable to DE-PDBs (137 against 132), however, it was able to find more unsolvable projections (66 against 48). Increasing the maximum allowed PDB size had varied effect, the total coverage dropped when it did not have any mutexes, and went up slightly when it had mutexes. Note that, we did not increase the maximum allowed pattern collection size. In other words, the total memory usage stayed the same and it was not utilised significantly better by increasing PDB size.

The reason why $H C_{B L}$ performs surprisingly well is because it stops once it finds an unsolvable projection, regardless of its score. This is especially clear in the second total coverage, where 49 of 64 instances were proven as unsolvable by finding an unsolvable projection. No 3-SAT, Pegsol or Tile was proved as unsolvable by encountering an unsolvable projection, and unsolvability was instead proven by exhaustive search. Note that iPDB performed significantly worse despite being similar to $H C_{B L}$, and found only 27 unsolvable projections. The poor performance of iPDB might be that it terminates its search too early since it does not see any significant heuristic improvement, and that this strategy does not work well on unsolvable instances. 


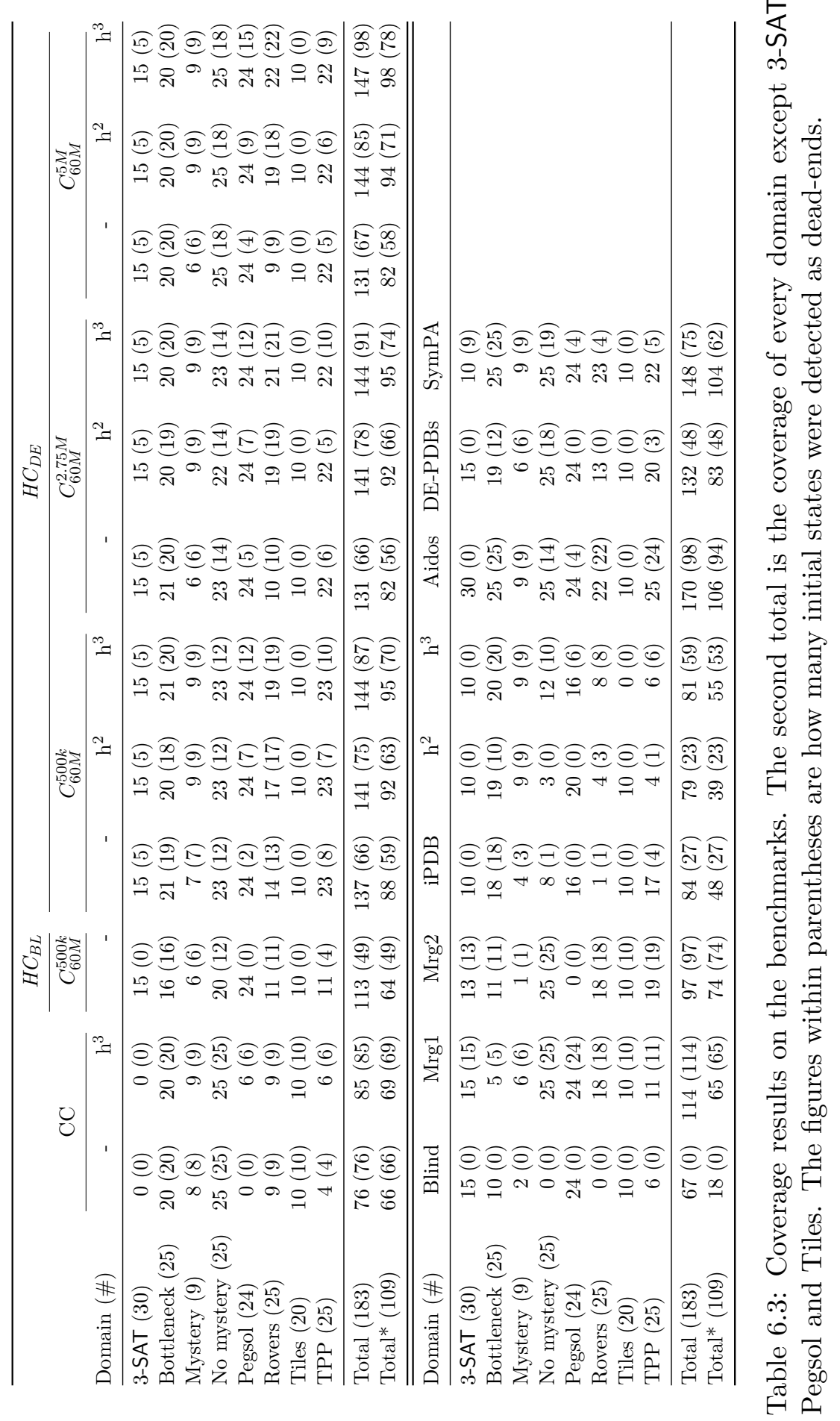




\subsubsection{Discussion}

An obvious critique against the statistical analysis done in Section 6.3 is that PDBs with the most dead-ends are not necessarily the best since there is no guarantee that those will have the most impact when pruning away reachable states. Suppose there exists a PDB with just one dead-end entry: the initial state. Obviously, there is no other PDB with a greater pruning impact since the search finishes immediately. However, this PDB would get a very low score by the scoring function $D E$ (but selected if found), and thus thus claiming that there are very few better pattern collections might not be true. The following theorem shows that the described PDB cannot exist unless the initial state is the only reachable state.

Theorem 6.2. Let $\Pi$ be a $\mathrm{SAS}^{+}$planning instance, and let $\left.\Pi\right|_{P}, P \subseteq$ $V(\Pi)$ be an unsolvable projection. Then for every reachable state $s$ of $\Pi, h^{P}(s)=\infty$.

Proof. Since $\left.\Pi\right|_{P}$ is unsolvable, then $h^{P}\left(\left.I(\Pi)\right|_{P}\right)=\infty$. This means that every state $s$ reachable from $\left.I(\Pi)\right|_{P}$ is also $h^{P}(s)=\infty$ (otherwise $h^{P}$ was computed incorrectly). Suppose there is a reachable state $s^{\prime}$ of $\Pi$ such that $h^{P}\left(\left.s^{\prime}\right|_{P}\right) \neq \infty$ (i.e. unreachable in $\left.\left.\Pi\right|_{P}\right)$. The proof of Theorem 2.8 essentially says that if a state is unreachable in a projection, then the matching states are unreachable in the original instance. This is easily shown by changing the goal of the original instance to a reachable matching state, which would bring forth the situation where the original instance is solvable but not a projection; an obvious contradiction. In other words, the assumption that $s^{\prime}$ is unreachable is contradictive, thus every reachable state of $\Pi$ is detected as a dead-end by $h^{P}$.

Theorem 6.2 is, perhaps, not surprising, but it explains a number of observations:

- PDBs of unsolvable projections can detect every reachable state as a dead-end, which means that we can expect PDBs of unsolvable projections to contain many dead-end entries. This is backed up by the data in Table 6.1, except for Bottleneck and Mystery, for which the average percentile ranks are a fair bit below 99 . This is possible since there might be states that are both unreachable and dead-ends, i.e. the state transition graph consists of more than 2 weakly connected components. Entries in a PDB for such states 
are, of course, pointless, and perhaps the scoring function for $H C_{D E}$ should check whether the state associated with a dead-end entry is reachable in the projection to avoid giving such PDBs an unsuitable high score. This is not a problem for $H C_{\text {Samples }}$ since it evaluate PDBs on reachable states.

- Theorem 6.2 also reveals an important property of PDBs: let $s$ be a state of the original instance that is detected as a dead-end by some PDB, then the very same PDB detects every state reachable from $s$ as a dead-end. This is trivially shown by replacing the initial state of the instance with $s$, thus Theorem 6.2 is applicable. Perhaps, most PDBs with a high percentage of dead-ends identified a state close to the initial state as a dead-end, and therefore also all subsequent states. This might also explain why the Tiles domain is difficult: it is possible to undo actions (reversible actions), hence, proving that one reachable state is a dead-end also proves that every reachable state is a dead-end. Solvable projections with only reversible actions cannot contain any reachable dead-end state since one would imply that every state is a dead-end, including goal states.

- Another possible critique against the statistical analysis in Section 6.3 is that we evaluated the methods on 'only' 20000 states, and that they were generated by using breadth-first search (instead of, for instance, depth-first search). We chose breadth-first search since detecting dead-ends close to the initial state often leads to pruning a large part of the state transition graph. Of course, there is no guarantee that this will happen. However, Theorem 6.2 says that if a PDB detects a dead-end state, then all states reachable from it are detected as dead-ends by the very same PDB. The problem with generating states using depth-first search is that it can give a misleading score, e.g. when a single PDB detects a large part of a search as dead-ends.

At this point, we have hopefully established that the scoring function $D E$ is a good way of measuring PDBs with respect to how well they prune away reachable states, and that $H C_{D E}$ finds PDBs with very high scores (Chapter 6.3). Two concerns that we have not discussed yet are: we did not use all of the IPC instances to evaluate $H C_{D E}$; and that the statistical analysis should have considered more and larger PDBs. Obviously, we believe that we would get a similar result, however, this 
kind of analysis is inherently computationally expensive and has to be restricted.

Based on the statistical analysis, we believe that there is little point in spending more time to construct a better pattern collection. Furthermore, since the pattern collections are often very good, we believe that further improvement is likely to come from enhancing patterns with other techniques such as mutexes. We showed that mutexes generated by $h^{m}$ had a tremendous impact on the number of dead-ends in PDBs, and it would be interesting to compare different mutex generation methods. Another possibility of improving PDBs is to simplify the instance such that the simplified instance is solvable if and only if the original instance is, e.g. redundant actions [HJ00] are actions that can be removed since they are replaceable by a sequence of other actions. DE-PDBs and $H C_{D E}$ without mutexes perform similarly, and a difference is the order they consider patterns. DE-PDBs is not as aggressive as $H C_{D E}$ since it considers patterns in the same way as consistency checking, perhaps they differ significantly w.r.t. pattern collection construction time.

It is worth noting that solvable instances might also contain deadends and therefore the proposed methods might be useful for such instances, e.g. Sokoban [PRB14]. Pattern collections can easily be tailored toward both heuristic guidance and dead-end detection; it is simply a matter of selecting different PDBs for either purpose. However, the cost is increased pattern collection construction time or increased memory usage. The latter might be addressed by compressing the PDBs [FKMH07]. 



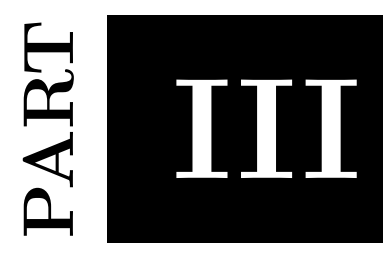

\section{Polytree Causal Graphs \& Variable Similarity}




\section{Overview of Part III}

We now take a short intermission in our enterprise to prove unsolvability, and consider instances with polytree causal graphs. In Chapter 7, we reveal two computationally tractable classes of instances, which both have polytree causal graphs and meet some additional restrictions. The notion of variable isomorphism was pivotal for finding the first class, and intuitively two variables are isomorphic if they behave in the same way in some optimal solution. If we know of an isomorphic pair, then we can remove one of the two variables without affecting solvability. If we also want to preserve optimal solution cost, then the action costs for the variables that we left intact need to be adjusted. We do precisely this for instances with polytree causal graphs, and identify a number of restrictions for when such a strategy yields a polynomial time algorithm. In Chapter 8, we use a variant of variable isomorphism to analyse unsolvable projections. We also identify another related tractable class through the idea of instance splitting, which splits the instance such that the original instance is solvable if and only if the smaller corresponding instances are. We construct a divide-and-conquer algorithm which runs in polynomial time when the instance meet certain restrictions.

We carried out an extensive statistical analysis of unsolvable projections in Chapter 5.3 and noted that unsolvable projections tend to have a significant number of variables in common. In Chapter 8 we ask the question: why those variables? We postulate that unsolvable projections tend to be composed of variables that play different roles, i.e they are not 'similar'. Isomorphic variables are, in some sense, extremely similar, and we devise a variable similarity measure to rate how similar two variables are. We then perform a statistical analysis and show that the variable similarity measure can differentiate between unsolvable and solvable projections quite well. This measure is then used to optimise consistency checking. 


\section{Chapter Seven \\ Tractable Classes}

One approach for constructing heuristic functions is to: (1) find a tractable (i.e. polynomial-time solvable) class of planning instances; (2) abstract the original instance to an instance in the tractable class and solve it; and (3) use the cost of the solution for the abstract instance as a heuristic value (cf. Helmert [Hel04]; Helmert, Haslum and Hoffman [HHH07]; Katz and Domshlak [KD10]). An ongoing quest in the planning community is to identify as many and as general tractable classes as possible, since having knowledge of these might prove invaluable for constructing efficient planners [KD08a]. A common approach to the problem of identifying tractable instances is to impose restrictions on the causal graph (cf., Brafman and Domshlak [BD03], Katz and Domshlak [KD07], or Giménez and Jonsson [GJ12]). In this chapter, we consider instances whose causal graphs are polytrees, i.e. acyclic graphs whose underlying undirected graphs are trees. PG is known to be NP-hard for instances whose causal graph is a directed path and having domain size $\geq 5$ [GJ09]. This implies that such instances must be avoided when searching for tractable fragments. A natural parameter when considering causal graphs is the diameter, that is, the length of the longest simple undirected path. Restrictions on the diameter have been used in previous work. For instance, Katz and Domshlak ([KD08b, KD10]) studied forks and inverted-forks while Katz and Keyder [KK12] studied hourglasses. In all these cases, the causal graph is a polytree with diameter $\leq 2$. Another parameter is the depth, namely, the length of the longest simple directed path. With this in mind, we use the following notation to define classes.

Definition 7.1. The classes $\overline{\mathcal{P}}_{k, d}^{i, o}$ and $\overrightarrow{\mathcal{P}}_{k, d}^{i, o}$ consist of instances that are restricted in the following way: (1) the causal graph is a polytree; (2) the indegree of the causal graph is bounded by a fixed constant $i$; 
(3) the outdegree of the causal graph is bounded by a fixed constant $o$;

(4) for $\overline{\mathcal{P}}_{k, d}^{i, o}$ the diameter is bounded by a fixed constant $d$, whilst for $\overrightarrow{\mathcal{P}}_{k, d}^{i, o}$ the depth is bounded by a fixed constant $d ;(5)$ and the domain size of every variable is bounded by $k$. We use the symbol $\infty$ to denote when the class is unrestricted in some regard.

Note that the only difference between the two classes is (4), i.e. whether we restrict the diameter or the depth of the causal graph. This is indicated by the line above the $\mathcal{P}$; no arrow head indicates that we restrict the diameter (undirected path), and an arrow head indicates that we restrict the depth (directed path). Section 7.2 shows that the class $\overline{\mathcal{P}}_{k, d}^{\infty, \infty}$ is tractable, and Section 7.3 shows that $\overrightarrow{\mathcal{P}}_{k, d}^{i, \infty}$ is tractable. In other words, the bound on the diameter is traded for a bound on the depth and a bound on the indegree. Note that a bounded depth still allows for an unbounded diameter.

\subsection{Preliminaries}

We briefly introduce and repeat some terminology that is used throughout this chapter. Consider a digraph $P=(V, E) . P$ is a polytree if it is acyclic and the underlying undirected graph is a tree. The diameter of $P$ is the longest shortest path in the underlying undirected graph, and the depth of $P$ is the longest shortest directed path in the underlying directed graph. A vertex $v \in V$ is a leaf if the degree of $v$ is exactly 1. Furthermore, we say that $v$ is an ingoing leaf if $(v, w) \in E$, and we say that $v$ is an outgoing leaf if $(w, v) \in E$, for some vertex $w \in V$. A vertex $v \in V$ is critical if it is connected to at least one leaf, and at most one non-leaf. The non-leaf connected to a critical vertex is its parent.

Planning instances whose causal graph is a polytree only have unary actions ( $a$ is unary if $|\operatorname{Domain}(\operatorname{eff}(a))|=1$ ), otherwise a cycle would have been introduced in the causal graph. We also define $A \dagger^{v}=\{a \in$ $A: v \in \operatorname{Domain}(\operatorname{pre}(a))\}$ and $A \dagger_{v}=\{a \in A: v \in \operatorname{Domain}(\operatorname{eff}(a))\}$. Additionally, the notation $\|x\|$ denotes how many bits are required to represent $x$ with a reasonable encoding. For example, $\|\Pi\|$ is how many bits we need to represent every variable (including its domain), every action, the initial state and the goal.

Lastly, we remind that the prevailconditions of an action $a$ are the preconditions that are still satisfied after applying the action, i.e. $a$ has a prevailcondition on $v$ if $v \in \operatorname{Domain}(\operatorname{pre}(a)) \backslash \operatorname{Domain}(\operatorname{eff}(a))$. 


\begin{tabular}{|c|c|c|c|}
\hline$d$ & 2 & fix. const & free \\
\hline 2 & COPG is in $\mathbf{P}$ & COPG is in $\mathbf{P}^{*}$ & PG is NP-h. ${ }^{* *}$ \\
\hline fix. const & COPG is in $\mathbf{P}$ & COPG is in $\mathbf{P}$ & PG is NP-h. \\
\hline free & PG is NP-h. ${ }^{* * *}$ & PG is NP-h. & PG is NP-h. \\
\hline
\end{tabular}

Table 7.1: The known results for $\overline{\mathcal{P}}_{k, d}^{\infty, \infty}$. The shaded cells are results that can be derived from the neighbouring cells, and the results in bold are the new results. (* Katz and Keyder [KK12]. ** Domshlak and Dinitz [DD01]. *** Giménez and Jonsson [GJ08].)

\subsection{Bounded Diameter}

The currently known results are presented in Table 7.1. We note that some of the previous work has used a slightly different set of restrictions and is more general, but the results are still applicable to the restrictions that we consider. These results left an open case: what happens if both the domain size and the diameter are bounded by arbitrary constants? We show that COPE is in $\mathrm{P}$ which immediately implies that COPG is in $\mathrm{P}$, too. This follows from the fact that the length of our optimal solutions are bounded by a polynomial in the instance size so we can use prefix search for generating cost-optimal solutions given that we have a polynomial time algorithm for COPE.

Let us compare our result with some other results for polytree causal graphs. Brafman and Domshlak [BD03] gave a polynomial time algorithm for instances with binary variables and where the causal graph is a polytree such that the indegree of every vertex is bounded by a constant. Giménez and Jonsson [GJ12] later generalised this result by giving an algorithm that, instead of restricting the indegree, restricts the number of prevailconditions. Note that a bound on the indegree implies a bound on the number of prevailconditions but not the other way round. Our algorithm is a bit different: it allows not only binary domains, but any domain size bounded by a constant and it allows the actions to have an unbounded number of prevailconditions. However, this flexibility comes at the expense of the diameter which must be bounded by a constant.

In this section, we devise an algorithm built on the notion of isomorphic variables. Intuitively, two variables are isomorphic if the structure of their domain transition graphs are isomorphic with regard to initial value, goal value, actions, prevailconditions, and so on. In other words, the two variables will (or can) behave exactly the same in 
every optimal solution, which allows us to combine them into a single variable. By combining isomorphic variables, we can gradually reduce the size of the initial instance, and this allows us to devise an algorithm that runs in polynomial time.

\subsubsection{Isomorphism}

Identifying isomorphic variables is the keystone of our algorithm; in fact, we will only need to take leaves into consideration. The basic idea is to look for isomorphic leaves that are connected to the same critical vertex and remove them in a systematic way. The algorithm will remove variables such that the set of critical vertices changes. The next lemma guarantees that we will not run out of critical vertices.

Lemma 7.2. Every tree consisting of at least three vertices has a critical vertex.

Proof. Let $G=(V, E)$ be a tree, and since $|V| \geq 3$ there exists a vertex $v \in V$ that is not a leaf. Remove all of the leaves of $G$ along with their connected edges, resulting in $G^{\prime}=\left(V^{\prime}, E^{\prime}\right)$. Since $v$ is not a leaf of $G$ then $v \in V^{\prime}$, i.e., $\left|V^{\prime}\right| \geq 1$. Per definition, every leaf of $G^{\prime}$ is a critical vertex of $G$ and there exists at least one leaf.

In graph-theoretic terms, two graphs $(V, E),\left(V^{\prime}, E^{\prime}\right)$ are said to be isomorphic if there exists a bijective function $\sigma: V \rightarrow V^{\prime}$ such that $(v, w) \in E$ if and only if $(\sigma(v), \sigma(w)) \in E^{\prime}$. However, we obviously need to take more structure into consideration when considering isomorphisms between variables in planning instances. This implies that we need a slightly more involved definition.

Before we define isomorphism we need the following defintions. Let $\omega=\left\langle a_{1}, \ldots, a_{n}\right\rangle$ be a sequence of actions, then $\mathcal{P}(\omega, v)=\left\langle\operatorname{pre}\left(a_{1}\right)[v]\right.$, $\left.\ldots, \operatorname{pre}\left(a_{n}\right)[v]\right\rangle$ denote the prevail- or preconditions $\omega$ has on the variable $v$. Also let $\mathcal{S}(\omega, v)=\left\langle I[v], \operatorname{eff}\left(a_{1}\right)[v], \ldots, \operatorname{eff}\left(a_{n}\right)[v]\right\rangle$ denote the values $v$ visits. If either the prevail-, precondition or effect is undefined for $v$ then the result is $u$, which symbolises an undefined value.

Definition 7.3. Let $\Pi=(V, A, I, G) \in \overline{\mathcal{P}}_{k, d}^{\infty, \infty}$. Two leaves $l_{1}, l_{2} \in$ $C G(\Pi)$ that are connected to the critical vertex $x$ are isomorphic if and only if there exists a bijective function $\sigma: D_{l_{1}} \rightarrow D_{l_{2}}$ such that:

1. $\operatorname{DTG}\left(l_{1}\right)$ is isomorphic to $\operatorname{DTG}\left(l_{2}\right)$ by $\sigma$,

2. $\sigma\left(I\left[l_{1}\right]\right)=I\left[l_{2}\right]$ and $\sigma\left(G\left[l_{1}\right]\right)=G\left[l_{2}\right]$, where if both $l_{1}, l_{2} \notin$ $\operatorname{Domain}(G)$ then the equality is true, 
3. $A \dagger^{l_{1}} \backslash A \dagger_{l_{1}}=A \dagger^{l_{2}} \backslash A \dagger_{l_{2}}$, and for every $a \in A \dagger^{l_{1}} \backslash A \dagger_{l_{1}}, \sigma\left(\operatorname{pre}(a)\left[l_{1}\right]\right)=$ $\operatorname{pre}(a)\left[l_{2}\right]$,

4. For every edge $\left(d, d^{\prime}\right) \in D T G\left(l_{1}\right)$ with label $L_{1},\left(\sigma(d), \sigma\left(d^{\prime}\right)\right) \in$ $\operatorname{DTG}\left(l_{2}\right)$ with label $L_{2}, L_{1}=L_{2}$, and the converse is true, and

5. For every $d, d^{\prime} \in D_{l_{1}}$, let $\omega_{l_{1}}, \omega_{l_{1}}^{\prime}$ be sequences of actions no longer than $\left|D_{l_{1}}\right|$ that take $l_{1}$ from $d$ to $d^{\prime}$, and $\omega_{l_{2}}, \omega_{l_{2}}^{\prime}$ be sequences of actions that take $l_{2}$ from $\sigma(d)$ to $\sigma\left(d^{\prime}\right)$ such that

- $\mathcal{P}\left(\omega_{l_{1}}, x\right)=\mathcal{P}\left(\omega_{l_{2}}, x\right)$,

- $\sigma\left(\mathcal{S}\left(\omega_{l_{1}}, l_{1}\right)\right)=\mathcal{S}\left(\omega_{l_{2}}, l_{2}\right)$,

- $\mathcal{P}\left(\omega_{l_{1}}^{\prime}, x\right)=\mathcal{P}\left(\omega_{l_{2}}^{\prime}, x\right)$, and

- $\sigma\left(\mathcal{S}\left(\omega_{l_{1}}^{\prime}, l_{1}\right)\right)=\mathcal{S}\left(\omega_{l_{2}}^{\prime}, l_{2}\right)$.

Then $R\left(c\left(\omega_{l_{1}}\right), c\left(\omega_{l_{1}}^{\prime}\right)\right)=R\left(c\left(\omega_{l_{2}}\right), c\left(\omega_{l_{2}}^{\prime}\right)\right)$, where: $R(x, y)=-1$ if $x<y ; R(x, y)=0$ if $x=y$; and $R(x, y)=1$ otherwise.

We note that the definition implicitly requires $l_{1}, l_{2}$ to be either both in- or outgoing from $x$. Condition 3 requires every action with an effect on $x$ to have the same prevailconditions on both $l_{1}, l_{2}$ (i.e., both are ingoing leaves), and condition 4 requires the actions in the leaves to have the same prevailconditions on $x$ (i.e., both are outgoing leaves). Condition 5 requires the costs of the actions for $l_{1}$ and $l_{2}$ to be 'compatible' with each other, i.e., the best actions to use to get to a particular value are the same no matter how $x$ behaves.

We will now see that there are explicit bounds on the number of non-isomorphic leaves. Define $\operatorname{IN}(x)$ and $\operatorname{OUT}(x)$ to be the sets of ingoing and outgoing leaves of a critical vertex $x$, respectively. We give a proof for out-leaves; the proof for in-leaves can be done in a similar way.

Lemma 7.4. Every critical vertex $x$ has at most

$$
f_{O}(m)=m(m+1) 2^{(m+1)^{3}}\left(\left((m+1)^{3}+1\right)^{m} !\right)
$$

non-isomorphic outgoing leaves, where $m=\max _{v \in\{x\} \cup \operatorname{Out}(x)}\left|D_{v}\right|$.

Proof. Let $l \in \operatorname{Out}(x)$. Every $a \in A \dagger_{l}$ is of the form $\{(x, \alpha),(l, \beta) \rightarrow$ $(l, \gamma)\}$ or $\{(x, \alpha) \rightarrow(l, \gamma)\}$, which means $m \cdot(m+1)$ possible preconditions and $m$ possible effects, thus $\left|A \dagger_{l}\right| \leq(m+1)^{3}$. A DTG consists of a subset of these actions, hence, there are at most $2^{(m+1)^{3}}$ different DTGs that $l$ 
can have. There are $m$ options for an initial value and $m+1$ options for a goal value (including no goal value). Furthermore, there are at most $\left(\left|A \dagger_{l}\right|+1\right)^{m}$ different sequences of actions of length no longer than $m$ and they can be sorted in at most $\left(\left|A \dagger_{l}\right|+1\right)^{m}$ ! different ways, which brings us to the final value $m(m+1) 2^{(m+1)^{3}}\left(\left((m+1)^{3}+1\right)^{m} !\right)$.

Lemma 7.5. Every critical vertex $x$ has at most $f_{I}(m)=f_{O}(m)(m+1)^{q}$ non-isomorphic ingoing leaves, where $q=\left|A \dagger_{x}\right|$ and $m=\max _{v \in\{x\} \cup \operatorname{IN}(x)}\left|D_{v}\right|$.

Proof. Since an ingoing leaf has no prevailconditions on other variables, the total number of different DTGs that it can have is less that or equal to $f_{O}(m)$ (see proof of Lemma 7.4). For each one of the $q$ actions, every ingoing leaf can have either no precondition on, or one precondition chosen from the $m$ values. Hence, there are $(m+1)^{q}$ different ways to set preconditions on all ingoing leaves for the $q$ actions that affect the critical node.

\subsubsection{Defoliation of Polytrees}

After having identified isomorphic leaves in the causal graph, our algorithm will remove them. This will be accomplished with two methods where we combine isomorphic leaves and then take the product of the remaining leaves and their correpsonding critical vertex. The idea behind this is that the combination step removes sufficiently many leaves and, thanks to that, the new product vertices will have domain sizes that grow in a controlled way. This method also guarantees that the number of vertices will decrease monotonically.

Definition 7.6. Let $\Pi=(V, A, I, G) \in \overline{\mathcal{P}}_{k, d}^{\infty, \infty}$ be an instance, $c$ be the cost function, and $v_{1}, v_{2} \in V, v_{1} \neq v_{2}$ be two isomorphic variables (i.e. leaves) by a function $\sigma$ such that both are connected to the same critical variable $x \in V$. The result of combining the two variables $v_{1}$ and $v_{2}$ is the instance $\Pi^{\prime}=\left(V^{\prime}, A^{\prime}, I^{\prime}, G^{\prime}\right)$ and cost function $c^{\prime}$ where:

- $V^{\prime}=\left(V \backslash\left\{v_{1}, v_{2}\right\}\right) \cup\left\{v_{3}\right\}$, where $v_{3} \notin V$,

- $D_{v_{3}}=D_{v_{1}}$,

\section{- Prevailconditions on $x$.}

Let $\lambda\left(a_{1}, a_{2}\right)=\operatorname{pre}\left(a_{1}\right) \rightarrow \operatorname{eff}\left(a_{1}\right)$, where $v_{1}$ is replaced with $v_{3}$ and the cost of the action is $c\left(a_{1}\right)+c\left(a_{2}\right)$. Then $A_{1}=$ $\left\{\lambda\left(a_{1}, a_{2}\right): a_{1} \in A \dagger_{v_{1}}, a_{2} \in A \dagger_{v_{2}}, \sigma\left(\operatorname{pre}\left(a_{1}\right)\left[v_{1}\right]\right)=\operatorname{pre}\left(a_{2}\right)\left[v_{2}\right]\right.$, 
$\left.\sigma\left(\operatorname{eff}\left(a_{1}\right)\left[v_{1}\right]\right)=\operatorname{eff}\left(a_{2}\right)\left[v_{2}\right], \operatorname{pre}\left(a_{1}\right)[x]=\operatorname{pre}\left(a_{2}\right)[x]\right\}$, where we interpret two undefined as equal,

- Prevailconditions on $v_{1}$ and $v_{2}$.

Let $\lambda(a)$ replace every occurance of $v_{1}$ in $a$ with $v_{3}$. Then $A_{2}=$ $\left\{\left\{(v, d) \in \operatorname{pre}(\lambda(a)): v \neq v_{2}\right\} \rightarrow \operatorname{eff}(\lambda(a)): a \in A \dagger_{x}\right\}$, where every action keep its cost, i.e. $c(a)$,

- $A^{\prime}=\left(A \backslash\left(A \dagger_{v_{1}} \cup A \dagger_{v_{2}} \cup A \dagger_{x}\right)\right) \cup A_{1} \cup A_{2}$,

- $I^{\prime}=\left.I\right|_{V \backslash\left\{v_{1}, v_{2}\right\}} \cup\left\{\left(v_{3}, I\left[v_{1}\right]\right)\right\}$, and

- $G^{\prime}=\left.G\right|_{V \backslash\left\{v_{1}, v_{2}\right\}} \cup \begin{cases}\left\{\left(v_{3}, G\left[v_{1}\right]\right)\right\} & \text { if } v_{1} \in \operatorname{Domain}(G) \\ \{\} & \text { otherwise }\end{cases}$

Intuitively, we replace $v_{2}$ by removing every occurrence of it and for every action $a$ affecting $v_{1}$ we adjust $c(a)$ to be the sum of the original cost and the cost of corresponding action for $v_{2}$. We can reuse $v_{1}$, but for clarity we use a new variable $v_{3}$. From now on, we extend the bijective function $\sigma$ to $A \dagger_{v_{1}}$ such that for every $a_{1} \in A \dagger_{v_{1}}, \sigma\left(a_{1}\right)=a_{2} \in$ $A \dagger_{v_{2}}$ where $\sigma\left(\operatorname{pre}\left(a_{1}\right)\left[v_{1}\right]\right)=\operatorname{pre}\left(a_{2}\right)\left[v_{2}\right], \sigma\left(\operatorname{eff}\left(a_{1}\right)\left[v_{1}\right]\right)=\operatorname{eff}\left(a_{2}\right)\left[v_{2}\right]$ and $\operatorname{pre}\left(a_{1}\right)[x]=\operatorname{pre}\left(a_{2}\right)[x]$.

Theorem 7.7. The cost of an optimal solution does not change by combining isomorphic variables.

Proof. Let $\Pi=(V, A, I, G) \in \overline{\mathcal{P}}_{k, d}^{\infty, \infty}$ be an instance and $c$ a cost function, let $v_{1}, v_{2} \in V$ be two isomorphic variables by a function $\sigma$, and let $v_{3}$, $\Pi^{\prime}$ be the resulting variable and instance by combining $v_{1}$ and $v_{2}$. We show that there is a correspondence between solutions of $\Pi$ and $\Pi^{\prime}$.

Let $\omega$ be an optimal solution for $\Pi$. Let $x$ be the parent of $v_{1}$ and $v_{2}$. We note that $\omega$ might use 'non-isomorphic' sequences of actions for $v_{1}$ and $v_{2}$ to reach their respective goal value, but by Definition 7.3 it is easy to show that we can replace either sequence with an isomorphic sequence of actions which does not increase the total cost of the solution. We construct $\omega^{\prime}$ from $\omega$ in the following way: first, remove every action in $A \dagger_{v_{2}}$ from $\omega$; second, replace every $a \in \omega \cap A \dagger_{v_{1}}$ with $\lambda(a, \sigma(a))$; finally, replace every $a \in \omega \cap A \dagger_{x}$ with the corresponding action $a^{\prime}$ from $A_{2}$ of Definition 7.6. Since $v_{1}$ and $v_{2}$ are isomorphic it is straightforward to show that $\omega^{\prime}$ is a solution for $\Pi^{\prime}$ and using condition 5 of Definition 7.3 , they both have the same cost. 
Note that the process is reversible. Let $\omega^{\prime}$ be a solution for $\Pi^{\prime}$, then we can construct a solution $\omega$ for $\Pi$ from $\omega^{\prime}$ in the following way: replace every $a \in \omega^{\prime} \cap A_{1}$ with $a_{1}$ and $a_{2}$ where $a_{1}$ and $a_{2}$ are isomorphic by $\sigma$ and $a=\lambda\left(a_{1}, a_{2}\right)$; and replace every $a^{\prime} \in \omega^{\prime} \cap A_{2}$ with the corresponding $a \in A \dagger_{x}$ (according to definition of $A_{2}$ in Definition 7.3). The rest of the actions remain unchanged. Therefore, the cost of an optimal solution for $\Pi$ and $\Pi^{\prime}$ is the same.

By combining leaves (with a common critical vertex) we can achieve the bounds given by Lemmata 7.4 and 7.5. After doing so we will construct a new variable whose domain is the product of all the leaves and the critical vertex. The product of two variables is a variable whose domain consists of possible states the two variables can be in, and an action for one of the original variables are then instantiated to several actions, one for each applicable 'state' in the domain of the new variable. The definition of product is very similar to synchronised product or the merge step from merge-and-shrink (M\&S). The result from merge of M\&S ignores the dependencies between variables that have not been merged, while Definition 7.8 maintains these dependencies. In other words, taking the product of two variables by Definition 7.8 changes the representation of the problem, but not the problem itself.

Definition 7.8. Let $\Pi=(V, A, I, G) \in \overline{\mathcal{P}}_{k, d}^{\infty, \infty}$, let $c$ be a cost function and let $v_{1}, v_{2} \in V$. The product of $v_{1}$ and $v_{2}$ with respect to $\Pi$ and $c$ is the instance $\Pi^{\prime}=\left(V^{\prime}, A^{\prime}, I^{\prime}, G^{\prime}\right)$ and cost function $c^{\prime}$ :

- $V^{\prime}=\left(V \backslash\left\{v_{1}, v_{2}\right\}\right) \cup\left\{v_{3}\right\}$

- $D_{v_{3}}=\left\{\left\{d_{1}, d_{2}\right\}: d_{1} \in D_{v_{1}}, d_{2} \in D_{v_{2}}\right\} \cup\{\{g\}\}$ (assume $D_{v_{1}} \cap$ $\left.D_{v_{2}}=\varnothing\right)$

- $A^{\prime}=A_{1} \cup A_{2} \cup A_{3} \cup A_{4} \cup A_{g}$ :

- The following allows the forthcoming definitions to be concise.

$$
\Delta(s, v)= \begin{cases}\{s[v]\} & \text { if } v \in \operatorname{Domain}(s) \\ D_{v} & \text { otherwise }\end{cases}
$$

where $s$ is a partial state and $v$ is a variable.

- Effect on $v_{1}$ :

$A_{1}=\left\{\left.\operatorname{pre}(a)\right|_{V \backslash\left\{v_{1}, v_{2}\right\}} \cup\left\{\left(v_{3},\left\{d_{1}, d_{2}\right\}\right)\right\} \rightarrow\left\{\left(v_{3},\left\{\operatorname{eff}(a)\left[v_{1}\right]\right.\right.\right.\right.$, $\left.\left.\left.\left.d_{2}\right\}\right)\right\}: a \in A \dagger_{v_{1}}, d_{1} \in \Delta\left(\operatorname{pre}(a), v_{1}\right), d_{2} \in \Delta\left(\operatorname{pre}(a), v_{2}\right)\right\}$, and the cost of every new action is $c(a)$, 
- Effect on $v_{2}$ :

$A_{2}$ is analogous to $A_{1}$ : swap $v_{1}$ with $v_{2}$ in the definition,

- Prevailconditions on $v_{1}$ and/or $v_{2}$ :

$A_{3}=\left\{\left.\operatorname{pre}(a)\right|_{A \backslash\left\{v_{1}, v_{2}\right\}} \cup\left\{\left(v_{3},\left\{d_{1}, d_{2}\right\}\right)\right\} \rightarrow \operatorname{eff}(a): a \in\right.$ $\left(A \dagger^{v_{1}} \cup A \dagger^{v_{2}}\right) \backslash\left(A \dagger_{v_{1}} \cup A \dagger_{v_{2}}\right)$, and the cost of every new action is $\left.c(a), d_{1} \in \Delta\left(\operatorname{pre}(a), v_{1}\right), d_{2} \in \Delta\left(\operatorname{pre}(a), v_{2}\right)\right\}$,

- No effect and no prevailcondition on both $v_{1}$ and $v_{2}$ : $A_{4}=\left\{a \in A \backslash\left(A \dagger^{v_{1}} \cup A \dagger^{v_{2}} \cup A \dagger_{v_{1}} \cup A \dagger_{v_{2}}\right)\right\}$, and every action keep its cost.

- New goal actions: $A_{g}=\left\{\left\{\left(v_{3},\left\{d_{1}, d_{2}\right\}\right)\right\} \rightarrow\left\{\left(v_{3},\{g\}\right)\right\}: d_{1} \in \Delta\left(G, v_{1}\right), d_{2} \in\right.$ $\left.\Delta\left(G, v_{2}\right)\right\}$, and the cost of every new goal action is 0 .

- $I^{\prime}=\left(\left.I\right|_{V \backslash\left\{v_{1}, v_{2}\right\}} \cup\left\{\left(v_{3},\left\{I\left[v_{1}\right], I\left[v_{2}\right]\right\}\right)\right\}\right.$

- $G^{\prime}=\left(\left.G\right|_{V \backslash\left\{v_{1}, v_{2}\right\}} \cup\left\{\left(v_{3},\{g\}\right)\right\}\right.$

Basically, the domain of the new variable is the set product of the domains of the first two variables, and a special value $g$ for the goal. For every action in $A(\Pi)$, we construct at $\operatorname{most} \max \left\{\left|D_{v_{1}}\right|,\left|D_{v_{2}}\right|\right\}^{2}$ new actions, and an additional $\left|A_{g}\right|$ for the goal variable.

Theorem 7.9. The cost of an optimal solution does not change by taking the product of two variables.

Proof. We give a brief overview without going into fine points of Definition 7.8. Let $v_{1}$ and $v_{2}$ be the variables of the product, and $v_{3}$ the resulting variable.

$(\Rightarrow)$ Let $\omega$ be an optimal solution for $\Pi$. Then we can construct a solution $\omega^{\prime}$ for $\Pi^{\prime}$ by simply applying the rules in Definition 7.8. For every action $a \in \omega$, iterating from start to end:

1. Action $a$ has an effect on $v_{1}$ or $v_{2}$. Add the action in the rule to the end of $\omega^{\prime}$. A single action might yield several actions, and in this case we can 'apply' the current $\omega$ ' to figure out which one of the actions is applicable. It is guaranteed by construction that one action is applicable.

2. Action $a$ has a prevailcondition on $v_{1}$ or $v_{2}$. Add the action in the rule to the end of $\omega^{\prime}$. Just like the previous case, the action might yield several actions and we can do the same to figure out which one we should use. 


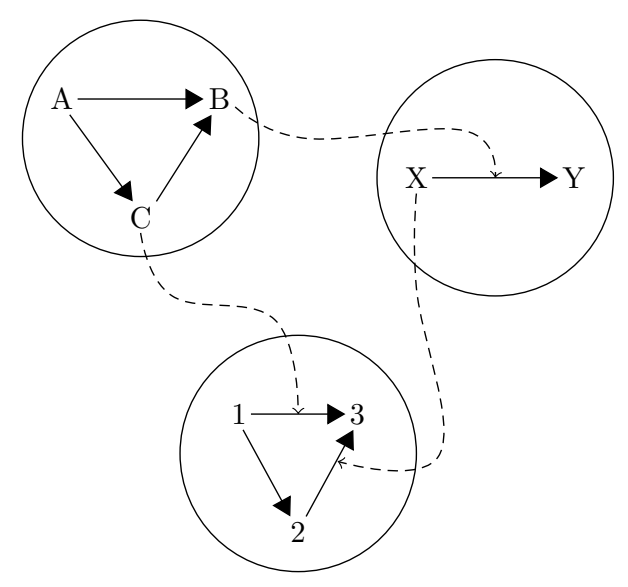

(a) Before the product.

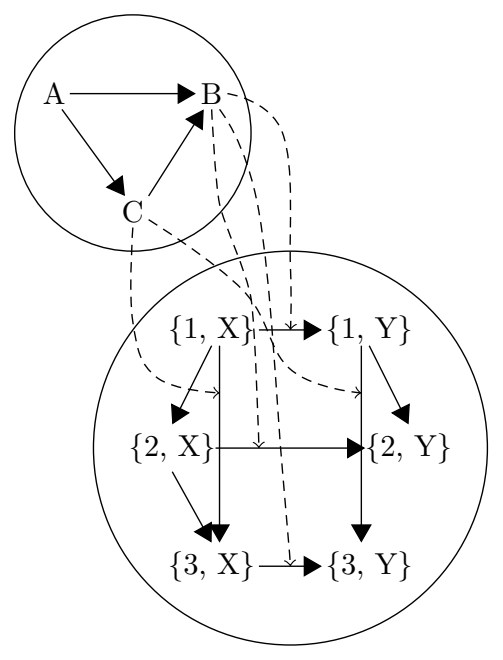

(b) After the product.

Figure 7.1: Circles are domain transition graphs, i.e. possible transitions between two values in the domain of the variable. Lines between values denote a precondition and an effect, and dashed lines to another line denote prevailconditions. Figure 7.1a presents the original instance, and Figure $7.1 \mathrm{~b}$ is the result of taking the product of the bottom and the right variable.

3. Action $a$ neither an effect nor prevailcondition on $v_{1}$ or $v_{2}$. Add action $a$ to the end of $\omega^{\prime}$.

If $v_{1}$ or $v_{2}$ is in $\operatorname{Domain}(G)$ then we need to add the corresponding action in $A_{g}$ to the end of $\omega^{\prime}$. By construction, $\omega^{\prime}$ is a solution, the replaced actions have the same cost and the cost of actions in $A_{g}$ is 0 . Therefore, $c(\omega)=c\left(\omega^{\prime}\right)$.

$(\Leftarrow)$ Let $\omega^{\prime}$ be an optimal solution for $\Pi^{\prime}$. It is straightforward to figure out which action in $A(\Pi)$ corresponds to an action $a \in \omega^{\prime}$. For actions with an effect or prevailcondition on $v_{1}$ or $v_{2}$, we simply have to find an action in $A(\Pi)$ that yields $a$ in the rule. If $a \in A_{g}$ then we skip it. Otherwise, we keep $a$ as is.

We illustrate the idea behind Definition 7.8 in Figure 7.1. The domain of the new variable is the cross product of each argument's domain; and the actions with a precondition or an effect on these variables are rewired to the new domain. The state transition graph 
before and after the product is essentially the same, with the exception that a goal value might need to be introduced (the figure does not illustrate this). We also make the following simple observation.

Lemma 7.10. The product of $n$ variables having domain size of at most $k$ results in a variable with domain size of at most $(k+1)^{n}$.

\subsubsection{The Algorithm}

We now present our algorithm, which can be found in Figure 7.2. The steps in the algorithm is roughly the following: (1) select a critical vertex $x,(2)$ identify the isomorphic leaves of $x,(3)$ combine the isomorphic leaves, and (4) take the product of the remaining leaves and the critical vertex. The resulting instance has strictly fewer variables so this process may be repeated in a recursive fashion. The algorithm terminates when there is two or fewer variables left, at which point the algorithm takes the product of the variables and uses Dijkstra's algorithm to compute the cost of an optimal solution.

Recall that, for a critical vertex $x$, the number of ingoing leaves for $x$ is bounded by a function in $\left|A \dagger_{x}\right|$. We need a constant bound on the number of leaves so this poses a problem. In other words, we must find a way to construct a set $\mathcal{R}_{x} \subseteq A \dagger_{x}$ that contains every action used in an optimal solution and $\left|\mathcal{R}_{x}\right|$ is bounded by a constant. Then, we can safely replace $A \dagger_{x}$ with $\mathcal{R}_{x}$. By knowing how many times a variables needs to change at most, we can construct the set $\mathcal{R}_{x}$.

Theorem 7.11. Let $\Pi \in \overline{\mathcal{P}}_{k, d}^{\infty, \infty}$ be an instance. The value of each variable in $\Pi$ changes at most $\mu$ times in every optimal solution, where

$$
\mu=t^{t j_{d \text { times }}^{t}} \quad t=(k(k+1))^{2}
$$

Proof. Let Change $_{\omega}(v)$ be the number of times that the value of variable $v$ changes in an optimal solution $\omega$. We claim that Change $_{\omega}(v) \leq p k(k+$ $1)^{p}+k$, where $p=\max \left\{\right.$ Change $\left._{\omega}\left(v^{\prime}\right):\left(v, v^{\prime}\right) \in C G(\Pi)\right\}$, and $p=0$ when there are no such variables $v^{\prime}$. Each one of the $v^{\prime}$ variables changes at most $p$ times, so each one of them can only have $p$ prevailconditions on $v$. Therefore, there are at most $(k+1)^{p}$ different sequences of prevailconditions on $v$. Variable $v$ takes at most $p$ different values in each sequence of prevailconditions, and there are at most $k$ changes between every two consecutive values (corresponding to a path in 
$D T G(v))$. This brings the number of changes up to $p k(k+1)^{p}$. Finally, there are at most $k$ actions needed to change the value of $v$ to $G[v]$ so Change $_{\omega}(v) \leq p k(k+1)^{p}+k$. By repeated use of this inequality and the fact that the diameter for $C G(\Pi)$ is bounded by $d$, a straightforward induction proof shows that Change $e_{\omega}(v) \leq \mu$.

Let Relevantactions $(\Pi, c, x)$ where $\Pi=(V, A, I, G), c: A \rightarrow \mathbb{N}$ and $x \in V$ be a function that computes the set $\mathcal{R}_{x}$ (with the properties we mentioned earlier) for the critical vertex $x$. Let $p$ be the parent of $x$ (we assume there exists one, otherwise it is trivial). The function does the following:

1. Let $\mathcal{R}_{x}=\varnothing$.

2. For every possible sequence of prevailconditions $P=\left\langle d_{1}, \ldots, d_{n}\right\rangle$, $d_{i} \in D_{p} \cup\{u\}$ (the value $u$ denotes an undefined prevailcondition), $n \leq \mu$ compute the cheapest sequence $\omega_{x}=\left\langle a_{1}, \ldots, a_{n}\right\rangle$ such that $\mathcal{P}\left(\omega_{x}, p\right)=P$. The cost associated with $\omega_{x}$ is the sum of $c\left(\omega_{x}\right)$ and the cost of the cheapest possible action sequence for the leaves with regard to the prevailconditions of $\omega_{x}$. The cheapest $\omega_{x}$ can be computed by simply trying all $\left|A \dagger_{x}\right|^{\mu}$ combinations. he cost of the cheapest possible action sequence for the leaves can be computed in the following way:

(a) For every $l \in \operatorname{IN}(x), \omega_{x}$ might have prevailconditions on $l$. To compute the cost of the cheapest path to the next prevailcondition, or goal value, we simply try every path in $\operatorname{DTG}(l)$ no longer than $\left|D_{l}\right|(\mu+1)$.

(b) For every $l \in \operatorname{Out}(x), \omega_{x}$ does not have any prevailconditions on $l$, but the other way around may happen. To compute the cost of the cheapest sequence of actions, we simply try every sequence for $l$ no longer than $\left|D_{l}\right|$ such that its prevailconditions can be satisfied by $\omega_{x}$.

We then set $\mathcal{R}_{x}$ to $\mathcal{R}_{x} \cup\left\{a_{1}, \ldots, a_{n}\right\}$.

3. Return $\mathcal{R}_{x}$.

If there exists a solution for the original $\Pi$, then $p$ has a sequence of prevailconditions on $x$ that is not longer than $\mu$ and we have computed the cheapest sequence of actions for it. An optimal solution (if there is one) is preserved by replacing $A_{x}$ with $\mathcal{R}_{x}$, which we will prove later.

The other functions occurring in the algorithm are: 
- CRiticalVertex(П) returns a variable that is a critical vertex in $C G(\Pi)$.

- CombineIsoVars takes the instance and a set of variables which are either ingoing leaves or outgoing leaves in the causal graph, and checks if they are pairwise isomorphic, and combine them if they are.

- Product takes an instance and a set of variables and returns a new instance in which the variables in the second argument are replaced by their product.

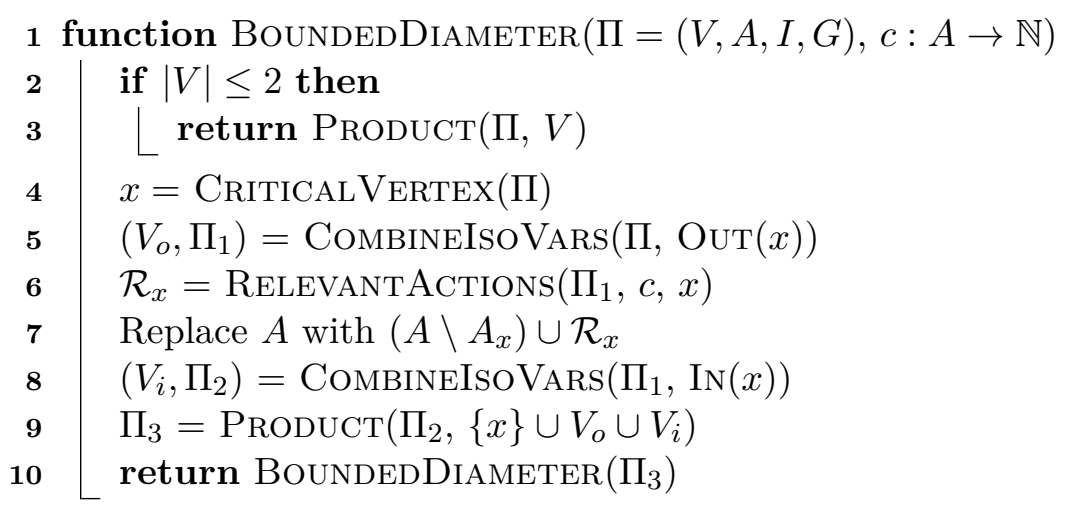

Figure 7.2: The devised algorithm, which outputs a new instance with a single variable of constant size.

\subsubsection{Correctness}

We begin by proving that algorithm BOUNDEDDIAMETER is correct. The groundwork has already been laid and the proofs are straightfoward. Firstly, we show that replacing actions with the ones computed by RELEVANTACTIONS does not change the cost of optimal solutions.

Lemma 7.12. The cost of an optimal solution does not change by replacing $A$ with $\left(A \backslash A \dagger_{x}\right) \cup \mathcal{R}_{x}$.

Proof. Let $\Pi=(V, A, I, G) \in \overline{\mathcal{P}}_{k, d}^{\infty, \infty}, c$ be a cost function, $\Pi^{\prime}=(V,(A)$ $\left.\left.A \dagger_{x}\right) \cup \mathcal{R}_{x}, I, G, c\right)$ and $p$ the parent of $x$. Let $\Pi$ have a solution $\omega$ and let $\alpha=\omega \cap A \dagger_{x}=\left\langle\alpha_{1}, \ldots, \alpha_{n}\right\rangle, a=\left\{a_{i}\right\}_{i=1}^{n}$ and $b=\left\{b_{i}\right\}_{i=1}^{n}$ such that $\operatorname{eff}\left(\alpha_{i}\right)[x]=a_{i}$ and $\operatorname{pre}\left(\alpha_{i}\right)[p]=b_{i}$. $\mathcal{R}_{x}$ contains enough 
actions for a locally optimal solution over $\{x\} \cup \operatorname{IN}(x) \cup \operatorname{OUT}(x)$ for any $(a, b)$. So, there exists a plan $\omega$ with actions in $\left(A \backslash A \dagger_{x}\right) \cup \mathcal{R}_{x}$ over $\{x\} \cup \operatorname{IN}(x) \cup \operatorname{OUT}(x)$ with the same cost as the part of $\omega$ over $\{x\} \cup \operatorname{IN}(x) \cup \operatorname{OUT}(x)$. Let

$$
\omega^{\prime}=\{a \in \omega: \nexists v \in\{x\} \cup \operatorname{IN}(x) \cup \operatorname{OUT}(x), v \in \operatorname{Domain}(\operatorname{eff}(a))\}
$$

Now, since both $\omega^{\prime}$ and $\omega$ require $x, p$ to take the values in $a, b$ for their execution, they can be combined together into a solution for $\Pi^{\prime}$ with the same cost as $\omega$.

We know that taking the product of two variables does not change the cost of optimal solutions, and next we show that the output of BOUNDEDDIAMETER does neither.

Theorem 7.13. Let $\Pi \in \overline{\mathcal{P}}_{k, d}^{\infty, \infty}$ be an instance. The cost of the optimal solution for BoundedDiameter( $\Pi)$ and $\Pi$ is the same.

Proof. According to Lemma 7.2, CRITICALVERTEX(П) returns a critical vertex $x$, which is connected to at least one leaf and the PRODUCT function reduces the number of variables by at least one. This means that the recursion stops after at most $|V|$ steps. Using Theorems 7.7, 7.9 and Lemma 7.12, the cost of the optimal solution for $\Pi$ and BoundedDiameter $(\Pi)$ are the same.

\subsubsection{Time Complexity}

We now turn our attention to the time complexity of algorithm PoLYTREE. The main result is proven in Theorem 7.18 which builds on Lemmata $7.14-7.17$. We remind the reader that $\|\Pi\|$ denotes the representational size of the instance $\Pi$.

Lemma 7.14. During the execution of BoundedDiameter( $\Pi)$, every $v \in V_{\Pi}$ is the output of CRITICALVERTEX at most once.

Proof. Assume that $x \in V$ is the output of a CRiticalVertex call. After $\operatorname{Product}\left(\Pi,\{x\} \cup V_{l}\right), x$ is a leaf. Hence, it cannot be a critical vertex anymore.

The fact that $\mathcal{R}_{x}$ contains at most $\mu$ actions for every pair $(a, b)$ of sequences of length at most $\mu$ over $D_{x}$ and $D_{p}$ including undefined, results in $\left|\mathcal{R}_{x}\right|<\mu^{2}\left|D_{x}\right|^{\mu}\left(\left|D_{p}\right|+1\right)^{\mu}$. After replacing $A \dagger_{x}$ with $\mathcal{R}_{x}$, we can use Lemma 7.5 and the bound on $\left|\mathcal{R}_{x}\right|$ to derive a new bound on 
the number of non-isomorphic ingoing leaves. Note that for instances in $\overline{\mathcal{P}}_{k, d}^{\infty, \infty}, \mu$ is a constant that only depends on $k$ and $d$. Now let $f(k)=(k+1)^{f_{O}(k)+f_{I}(k)+1}$ (where $f_{I}$ and $f_{O}$ are the functions defined in Lemmata 7.4 and 7.5, respectively) and let $f^{n}=f \circ f^{n-1}$ denote the $n$-th iteration of $f$.

Lemma 7.15. Let $y$ be the new variable created by PRoduct $(\Pi$, $\{x\} \cup V_{o} \cup V_{i}$ ) (where $V_{o}$ is the outgoing leaves connected to $x$ and $V_{i}$ are the ingoing). Then $\left|D_{y}\right| \leq f(m)$ where $m=\max \left\{\left|D_{v}\right|: v \in\right.$ $\left.\{x, p\} \cup V_{o} \cup V_{i}\right\}$ and $p$ is the parent of $x$.

Proof. This is straightforward according to Lemmata 7.4, 7.5, 7.10 and the fact that $f_{I}$ and $f_{O}$ are strictly increasing.

We now show that the domain of the final variable is bounded by a constant.

Lemma 7.16. $\left|D_{\text {BoundedDiameter( } \Pi)}\right| \leq f^{d}(k)$ for $\Pi \in \overline{\mathcal{P}}_{k, d}^{\infty, \infty}$.

Proof. Let $v$ be the variable in BoundedDiameter( $\Pi)$ and assume to the contrary that $\left|D_{v}\right|>f^{d}(k)$. Going one step back and using Lemma 7.15 , either one of the multiplied vertices or the parent of $v$ must have had a domain size larger than $f^{d-1}(k)$. According to Lemma 7.14, $v$ has not been a critical vertex before so it has a domain size of at most $k$. Therefore, there exists a vertex among the leaves or the parent of $v$, call it $v_{1}$, such that $\left|D_{v_{1}}\right|>f^{d-1}(k)$. Again, going one step back, there exists a vertex among the leaves or the parent of $v_{1}$, call it $v_{2}$, such that $\left|D_{v_{2}}\right|>f^{d-2}(k)$. Continuing this, we reach a vertex, $v_{d}$, such that $\left|D_{v_{d}}\right|>k$. Since the domain size of $v_{d}$ is greater than $k$, it cannot be a variable in the original instance $\Pi$, so we must have another vertex, call it $v_{d+1}$, that was either a leaf for $v_{d}$ or its parent if we go back again. This means that there is a path of length $d+1$ from $v$ to $v_{d+1}$ in the polytree, but the causal graph for $\Pi$ is supposed to be a tree with a diameter of $d$ and we have a contradiction.

Since every variable's domain is bounded by a constant during every step of the algorithm, we can guarantee that RELEVANTACTIONS runs in polynomial time.

Lemma 7.17. RelevantACTiOns runs in polynomial time.

Proof. As a result of Lemma 7.16, the domain size of all variables is bounded by $f^{d}(k)$ throughout the algorithm. It is not hard to see that the algorithm runs in polynomial time by combining this fact with the fact that $\mu$ is a constant. 
Finally, we show that the algorithm runs in polynomial time and thus the class is in $\mathbf{P}$.

Theorem 7.18. $\operatorname{COPE}\left(\overline{\mathcal{P}}_{k, d}^{\infty, \infty}\right)$ is in $\mathbf{P}$.

Proof. Let $\Pi=(V, A, I, G) \in \overline{\mathcal{P}}_{k, d}^{\infty, \infty}$ be an instance where $c$ is the cost function. The functions appearing in the algorithm run in polynomial time. In particular, one may note COMBINEIsoVARS runs in polynomial time since Lemma 7.16 implies that the variable domains are of constant size which implies that the isomorphism check can be performed in polynomial time. Hence, according to Lemma 7.14, BoundedDiA$\operatorname{Meter}(\Pi)$ runs in polynomial time, too. Finally, applying Dijkstra's algorithm to the resulting DTG does not change the overall complexity due to Lemma 7.16 and the fact that the action weights are bounded by a polynomial (that only depends on $k$ and $d$ ) in $\|\Pi\|$.

In order to use prefix search to construct an optimal solution for the original instance $\Pi$ we need an upper bound on the solution length that is less than some polynomial $p(\|\Pi\|)$. After running the algorithm on an instance $\Pi$, we get a new instance $\Pi^{\prime}$. Let $\omega$ be an optimal solution for $\Pi^{\prime}$. We know that the cost of an optimal solution for $\Pi$ is $c(\omega)$, but we do not have an upper bound on the length of it. Note that every action in $\Pi^{\prime}$ represents at most $|V|$ actions in $\Pi$, where $V$ is the variable set of $\Pi$, because the algorithm combines at most $|V|$ variables. Furthermore, $|\omega|$ is bounded by $f^{d}(k)$. Hence, a loose polynomial upper bound on the length of an optimal solution for $\Pi$ is $f^{d}(k) \cdot|V|$.

\subsubsection{Discussion}

\section{Exploiting Isomorphism}

Consider the class of instances $\Theta$ having fork causal graphs and domain size bounded by $k$. A directed graph $(V, E)$ is a fork if there exists a vertex $r \in V$ such that every $(u, v) \in E \Leftrightarrow u=r$. Definition 7.3 tells us when two leaves in $V(\Pi)$ are isomorphic. Since the domain size is bounded by $k$, then the number of non-isomorphic leaves is bounded by a constant $c$. This bound can be exploited by variable projection in the following way. As we have discussed extensively in Part II, if the projection of $\Pi$ onto $V^{\prime} \subseteq V(\Pi)$ is unsolvable, then so is $\Pi$. Suppose $V^{\prime}$ contains two isomorphic variables $l_{1}, l_{2}$. Clearly, removing $l_{1}$ or $l_{2}$ from $V^{\prime}$ would not make the projection solvable. Thus, it is pointless of to choose $V^{\prime}$ such that $\left|V^{\prime}\right|>c+1$ if one wants to check unsolvability. 
With this in mind, we can use the consistency checking algorithm for solving $\operatorname{PE}(\Theta)$ : (1) enumerate all variable sets of size $c,(2)$ for every such variable set project the instance onto it and solve it, (3) if it is unsolvable then report that the original instance is unsolvable, and (4) if all variable sets lead to solvable instances, then report that the instance is solvable. We see that this procedure is a sound and complete method for $\operatorname{PE}(\Theta)$ and it trivially runs in polynomial time. In fact, this is the first example of a non-trivial class of planning instances where consistency checking yields a polynomial-time algorithm that is simultaneously sound and complete.

This simple idea can, of course, be generalised. Up to now, we have only discussed isomorphic leaves but it is possible to generalise the definition to isomorphic subforks and more general substructures. While isomorphic variables would most likely be rare in practice, the observation that projecting onto a set containing isomorphic variables is pointless might hint that the inclusion of 'similar' variables should be avoided. Consequently, it might be interesting to measure how similar two variables are. We speculate that choosing a set whose variables are 'dissimilar' are the most useful for proving that an instance is unsolvable. We investigate this idea in the context of consistency checking in Chapter 8. We also speculate that this may, at least partially, explain why methods such as merge-and-shrink [HHH07] have been successful for identifying unsolvable instances [HKT14]. The way merge-and-shrink choses variables to merge may somehow correlate to their dissimilarity.

\section{The Algorithm}

Many polynomial-time algorithms for planning under polytree causal graphs share a common problem: the degree of the polynomials are very high, cf., Giménez and Jonsson [GJ12] and Katz and Keyder [KK12]. The presented algorithm is, unfortunately, plagued with the very same problem. We believe that our algorithm can be vastly improved in at least two different ways: (1) the upper bound on the number of times a variable has to change is very pessimistic and (2) the isomorphism checking procedure is based on blind exhaustive enumeration. However, such improvements do not change the idea of enumerating many different paths in the DTGs that our and the previously mentioned algorithms are based upon. A completely new idea may be needed in order to obtain significantly lower complexity figures.

We observe that the high degree of the polynomial stems from 
one single step, RELEVANTACTIONS, which is needed because we allow causal graphs with unbounded indegree. If the indegree is bounded by a constant, then after merging isomorphic outgoing leaves, the number of actions affecting the critical vertex is bounded by a constant. Hence, we can skip RELEVANTACTIONS and the time complexity of the algorithm is instead $\mathcal{O}\left(\|\Pi\|^{4}\right)$, which is dramatically better. This might hint that imposing a restriction on the indegree might be necessary to, for example, construct efficient heuristics based on polytree causal graphs. Furthermore, Katz and Domshlak [KD09] implemented and evaluated an admissible heuristic based on forks and inverted-forks, which the class studied in this thesis generalises. To overcome the problem that their heuristic was computationally quite expensive, they precompiled values and stored them in a database. The very same idea may be useful for a heuristic based on our class.

\subsection{Bounded Depth}

A more general restriction of diameter is depth. Whilst a bounded diameter implies a bounded depth, the opposite is not true. In this section we consider a bound on the depth instead of the diameter, and show that $\overrightarrow{\mathcal{P}}_{k, d}^{i, \infty}$ is tractable. That is, our algorithm requires a bounded indegree, which was not necessary for $\overline{\mathcal{P}}_{k, d}^{\infty, \infty}$. The algorithm presented in this section is built on the notion of splitting an instance into several independent instances. It is obvious that when we have several weakly connected components in the causal graph, then we can project the original instance onto each of them and solve them independently. The solutions for the projections can then be interleaved to construct a solution for the original instance. In other words, we fabricate weakly connected components in the causal graph by instance splitting, and then use a divide-and-conquer strategy to construct a solution (if one exists). As with most divide-and-conquer algorithms, it is desirable to have subproblems of about equal size, and in our case this means that the weakly connected components should have about the same number of variables.

Throughout this section, we will use the following assumption and notation. We assume, without loss of generality, that there does not exist two actions $a_{1}, a_{2}$ such that $\operatorname{pre}\left(a_{1}\right)=\operatorname{pre}\left(a_{2}\right)$ and $\operatorname{eff}\left(a_{1}\right)=\operatorname{eff}\left(a_{2}\right)$, but $c\left(a_{1}\right) \neq c\left(a_{2}\right)$. A weakly connected component is a subset of vertices that induces a subgraph such that there is an undirected path between any two vertices. We use the notation $\operatorname{wcc}(G)$ where $G$ is a graph to 
denote the weakly connected components in $G$ (i.e. is set of sets of vertices).

\subsubsection{Instance Splitting}

A crucial part of the forthcoming algorithm is how to decide on a variable to split the instance in; this must be done with care to prevent that some weakly connected component in the causal graph consists of the majority of the variables.

Theorem 7.19. Let $\Pi \in \overrightarrow{\mathcal{P}}_{\infty, \infty}^{\infty, \infty}$ be a planning instance with variable set $V$. There exists a split variable $v \in V$ which splits the causal graph $C G(\Pi)$ into weakly connected components $C_{1}, \ldots, C_{j}$ such that $\left|C_{i}\right| \leq|V| / 2$ for all $i, 1 \leq i \leq j$.

We say that every variable with this property is a split variable. The existence of such a variable is guaranteed [Vyg11] and can be computed in polynomial time. For instance, one could simply try to remove each variable from the original instance and write down the size of the largest component, and then return the variable that gave the smallest largest component. This algorithm runs in time $\mathcal{O}\left(|V|^{3}\right)$.

Once we have decided on a split variable, we split the instance. To do this we introduce a copy of the split variable into every newly introduced weakly connected component in the causal graph. Of course, this is insufficient since solutions for the projections onto these weakly connected components might use different actions for the copies, and to synchronise them we decide on a specific action sequence beforehand that every solution must use.

Definition 7.20. Let $\Pi=(V, A, I, G) \in \overrightarrow{\mathcal{P}}_{\infty, \infty}^{\infty, \infty}$ be a planning instance, $v \in V$ be a variable, and $S=\left\langle a_{1}^{v}, \ldots, a_{m}^{v}\right\rangle$ be a sequence of actions, where $a_{n}^{v} \in A \dagger_{v}$ for $1 \leq n \leq m$. Removing $v$ from $C G(\Pi)$ results in $j$ new weakly connected components, denoted $C_{1}, \ldots, C_{j}$. Splitting the instance $\Pi$ in variable $v$ by $S$ yields the instance $\operatorname{Split}(\Pi, S, v)=$ $\left(V^{\prime}, A^{\prime}, I^{\prime}, G^{\prime}\right)$ where:

- $V^{\prime}=\left\{v_{1}, \ldots, v_{j}\right\} \cup V \backslash\{v\}$, where $v_{1}, \ldots, v_{j}$ are new variables

- $A^{\prime}=\bigcup_{i=1}^{j}\left\{a_{1}^{v_{i}}, \ldots, a_{m}^{v_{i}}\right\} \cup \bigcup_{a \in A} \lambda(a)$, where $a_{1}^{v_{i}}, \ldots, a_{m}^{v_{i}}$ are new actions

- $I^{\prime}=\left\{\left(v_{1}, 0\right), \ldots,\left(v_{j}, 0\right)\right\} \cup I \backslash\{(v, I[v])\}$ 
- $G^{\prime}=\left\{\left(v_{1}, m\right), \ldots,\left(v_{j}, m\right)\right\} \cup G \backslash\{(v, G[v])\}$

The domain of every variable $v_{i} \in\left\{v_{1}, \ldots, v_{j}\right\}$ is $\{0, \ldots, m\}$, the actions affecting it are $a_{1}^{v_{i}}, \ldots, a_{m}^{v_{i}}$, and the definition of every action $a_{n}^{v_{i}}, 1 \leq$ $n \leq m, 1 \leq i \leq j$ is:

- $a_{n}^{v_{i}}=\left\{\left(v_{i}, n-1\right)\right\} \cup\left\{\left(v^{\prime}, d^{\prime}\right) \in \operatorname{pre}\left(a_{n}^{v}\right): v^{\prime} \in C_{i}\right\} \rightarrow\left\{\left(v_{i}, n\right)\right\}$

- $c\left(a_{n}^{v_{i}}\right)=c\left(a_{n}^{v}\right)$

Note that for every $i$, the action $a_{n}^{v_{i}}$ only has preconditions on variables in 1 weakly connected component in the causal graph. However, collectively, they have the same preconditions as the original action. (It is safe to do this since all newly introduced actions must be in every solution and in the same order.) The idea of $\lambda$ is to remap the prevailconditions on $v$ to the newly introduced variables. Let $C_{i}$ be the component that contains the variable that $a$ has an effect on, then $\lambda(a)$ is defined as follows.

$$
\lambda(a)= \begin{cases}\varnothing & \text { if } v \in \operatorname{Domain}(\operatorname{eff}(a)) \\ \{a\} & \text { if } v \notin \operatorname{Domain}(\operatorname{pre}(a)) \cup \operatorname{Domain}(\operatorname{eff}(a)) \\ \left\{a_{1}, \ldots, a_{k}\right\} & \text { otherwise }\end{cases}
$$

Where:

- $\mathcal{C}(S, v, d, I)=\sum\{1: s[v]=d, s \in\{\operatorname{eff}(a): a \in A\} \cup\{I\}\}$,

- $\mathcal{N}\left(\left\langle a_{1}^{v}, \ldots, a_{m}^{v}\right\rangle, l, v, d, I\right)= \begin{cases}0 & \text { if } l=1 \text { and } I[v]=d \\ & \text { where } l=\mathcal{C}\left(\left\langle a_{1}^{v}, \ldots, a_{n}^{v}\right\rangle, v, d, I\right) \\ & \text { and } \operatorname{eff}\left(a_{n}^{v}\right)[v]=d, \text { otherwise }\end{cases}$

- $k=\mathcal{C}(S, v, \operatorname{pre}(a)[v], I)$, and

- $a_{l}=\left\{\left(v_{i}, \mathcal{N}(S, l, v, \operatorname{pre}(a)[v], I)\right)\right\} \cup\left\{\left(v^{\prime}, d^{\prime}\right) \in \operatorname{pre}(a): v^{\prime} \neq v\right\} \rightarrow$ $\operatorname{eff}(a), c\left(a_{l}\right)=c(a)$, for $1 \leq l \leq k$.

The function $\mathcal{C}$ denotes how many times a value $d \in D_{v}$ is visited by the sequence $S$ and the initial state. The function $\mathcal{N}$ gives the position of the action in $S$ that visits the given value for the $l$ :th time.

Figure 7.3 illustrates various aspects of splitting: the need of a predefined action sequence for the split variable; introduction of copies of the split variable and actions with prevailconditions on it; and the goal of fabricating more weakly connected components in the causal 


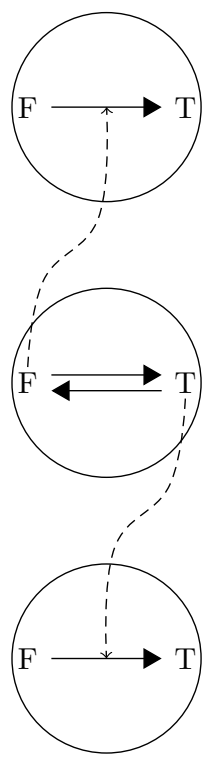

(a) Before the split.

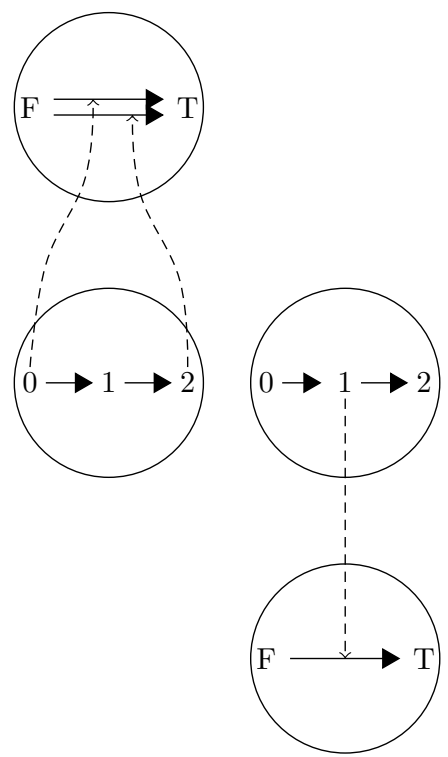

(b) After the split.

Figure 7.3: Circles are domain transition graphs. Lines between values denote a precondition and an effect, and dashed lines to another line denote prevailconditions. The initial instance consists of 3 binary variables. The instance was split in the middle variable by actions that changes the value from $F$ to $T$, and back to $F$. The resulting instance consists of 4 variables, whereas two new variables are ternary; and there are two actions that can change the value of the top variable, one for each middle value that represent the old prevailcondition. 
graph. Once we have split an instance and found solutions for each independent projection, we interleave them in the following way. Let $\omega=\left\langle a_{1}, \ldots, a_{n}\right\rangle, \omega^{\prime}=\left\langle a_{1}^{\prime}, \ldots, a_{m}^{\prime}\right\rangle$ be two sequences of actions, then $\omega\|\|$ $\omega^{\prime}=\left\langle a_{1}, \ldots, a_{n}, a_{1}^{\prime}, \ldots, a_{m}^{\prime}\right\rangle$. To simplify our algorithm, interleave also supports the special value 'Unsolvable': $\omega\|\mid\|$ Unsolvable $=$ Unsolvable, or Unsolvable $\| \mid \omega^{\prime}=$ Unsolvable. In other words, if any argument is 'Unsolvable', then so is the result.

Theorem 7.21. Let $\Pi \in \overrightarrow{\mathcal{P}}_{\infty, \infty}^{\infty, \infty}$ be a SAS ${ }^{+}$instance with cost function $c$. There exists a solution with total cost $t$ for $\Pi$ if and only if there exists a solution with $\operatorname{cost} t+c(S) \cdot(|\operatorname{wcc}(\operatorname{Split}(\Pi, S, v))|-|\operatorname{wcc}(\Pi)|)$ for Split $(\Pi, S, v)$ for every variable $v \in V(\Pi)$ and some action sequence $S$ with effects only on $v$.

Proof. $(\Rightarrow)$ Let $\omega=\left\langle a_{1}, \ldots, a_{m}\right\rangle$ be a solution for $\Pi$ such that $c(\omega)=t$. Let $S=\left\langle a_{1}^{v}, \ldots, a_{p}^{v}\right\rangle$ be every action in $\omega$ that are in $A \dagger_{v}$, in the same order. By Definition 7.20 the instance Split( $\Pi, S, v)$ has replaced the variable $v$ by $j=|\operatorname{wcc}(\operatorname{Split}(\Pi, S, v))|-|\operatorname{wcc}(\Pi)|+1$ new variables $v_{1}, \ldots, v_{j}$ and replaced every action that either had an effect or a prevailcondition on $v$ with actions that has an effect or a prevailcondition on one of the variables that replaced $v$. In order to construct a solution $\omega^{\prime}$ for $\operatorname{Split}(\Pi, S, v)$, we can replace every action $a$ in $\omega$ with a sequence of actions:

\section{- Action $a$ has a prevailcondition on $v$ :}

Let $v_{i}$ be the variable that replaced $v$ in the weakly connected component that also contains the variable $a$ has an effect on. Let $l$ be the number of times pre $(a)[v]$ is visited before $a$ in $\omega$. Then, replace $a$ with the action $a^{\prime}$ that has $\operatorname{pre}\left(a^{\prime}\right)\left[v^{\prime}\right]=l$ and $\operatorname{eff}(a)=\operatorname{eff}\left(a^{\prime}\right)$. This action exists since we based our split on $\omega$.

\section{- Action $a$ has an effect on $v$ :}

Let $n$ be the number of actions with an effect on $v$ in $\omega$ before $a$. Then, replace $a$ with the introduced actions $a_{n}^{v_{1}}, \ldots, a_{n}^{v_{j}}$ that has the precondition $\operatorname{pre}\left(a_{n}^{v_{i}}\right)\left[v_{i}\right]=n$ and $\operatorname{effect} \operatorname{eff}\left(a_{n}^{v_{i}}\right)\left[v_{i}\right]=n+1$.

\section{- Action $a$ has no prevailcondition nor effect on $v$ :}

Do not replace $a$, i.e. keep $a$.

We only replace actions that has an effect on $v$ with several actions, one for every introduced weakly connected component in the causal graph. The sequence $S$ consists of such actions and thus $c\left(\omega^{\prime}\right)=t+(j-1) c(S)$. 
$(\Leftarrow)$ Let $\omega$ be a solution for $\operatorname{Split}(\Pi, S, v)$ with cost $t$, where $S=$ $\left\langle a_{1}, \ldots, a_{m}\right\rangle$. Let $j=|\operatorname{wcc}(\operatorname{Split}(\Pi, S, v))|-|\operatorname{wcc}(\Pi)|+1$ be the number of variables introduced by the split. In other words, the weakly connected components $C_{1}, \ldots, C_{j}$ were introduced by the split. There might be additional weakly connected components that were part of the original instance, thus we do not need to consider them. Let $\omega_{i}$ be the part of $\omega$ that solves $\left.\Pi\right|_{C_{i}}$ (i.e. every action that has an effect on any variable in $C_{i}$ ). Note that $\omega_{1}\|\| \cdots \| \omega_{j}$ is a solution for $\operatorname{Split}(\Pi, S, v)$ since every solution only affects the variables in its component and can be interleaved in any way. Let $a_{n}^{v_{i}}, 1 \leq n \leq m, 1 \leq i \leq j$ be the actions that replaced $a_{n}$ for $v_{i}$. Another solution for $\operatorname{Split}(\Pi, S, v)$ is to interleave $\omega_{1}, \ldots, \omega_{j}$ such that the actions $a_{n}^{v_{1}}, \cdots, a_{n}^{v_{j}}$ are grouped up:

$$
\ldots, a_{1}^{v_{1}}, a_{1}^{v_{2}}, \cdots, a_{1}^{v_{j}}, \ldots, a_{n}^{v_{1}}, a_{n}^{v_{2}}, \cdots, a_{n}^{v_{j}} \ldots, a_{m}^{v_{1}}, a_{m}^{v_{2}}, \cdots, a_{m}^{v_{j}}, \ldots
$$

In other words, for every $n$, there are no other actions in between the actions $a_{n}^{v_{1}}, \cdots, a_{n}^{v_{j}}$, but with other actions in between these subsequences. To construct a solution $\omega^{\prime}$ for $\Pi$, we replace these subsequences, and actions with a prevailcondition on a new variable, with one action:

- Subsequence $a_{n}^{v_{1}}, \ldots, a_{n}^{v_{j}}$ :

Replace subsequence with action $a_{n}$.

- Subsequence $a$, where $a$ has a prevailcondition on $v_{i}$ :

Let $\omega_{i}$ be the sequence that contains $a$, and let $l$ be the number of actions with an effect on $v_{i}$ before $a$ in $\omega_{i}$. Then, replace $a$ with the action $a^{\prime}$ that has $c\left(a^{\prime}\right)=c(a), \operatorname{eff}(a)=\operatorname{eff}\left(a^{\prime}\right)$, and $\operatorname{pre}\left(a^{\prime}\right)=$ $\operatorname{pre}\left(\left.a\right|_{C_{i}}\right) \cup\left\{\left(v, \operatorname{eff}\left(a_{l}\right)[v]\right)\right\}$ or $\operatorname{pre}(a)=\operatorname{pre}\left(\left.a\right|_{C_{i}}\right) \cup\{v=I[v]\}$ if $l=0$. This action exists per definition.

- Subsequence $a$, where $a$ has no prevailcondition nor effect on any $v_{i}$ :

Do not replace $a$, i.e. keep $a$.

We replace the actions that were introduced by the split with the corresponding action that they replaced. Clearly,

$$
c\left(\omega^{\prime}\right)=t-(j-1) c(S)
$$

The proof of Theorem 7.21 specifies how to construct a solution for the original instance based on a solution for a split instance. There are no time-consuming steps and they can easily be performed in polynomial time, and the algorithm we present next is very much inspired by this proof. 


\subsubsection{Algorithm}

The input to the algorithm is a planning instance $\Pi \in \overrightarrow{\mathcal{P}}_{k, d}^{i, \infty}$, and the idea of the algorithm is as follows: (1) compute a variable $v$ to split in; (2) compute an upper bound of how many times $v$ might change value for any optimal solution; (3) for every possible sequence of actions $S$ that only affects $v$, we split $\Pi$ in $S$, perform recursive calls, and construct a solution for $\Pi$; and (4) return the solution with the smallest cost. Furthermore, if $\Pi$ only consists of 4 variables or less, then we simply solve it directly with some optimal and complete planner.

Theorem 7.22. Let $\Pi=(V, A, I, G) \in \overrightarrow{\mathcal{P}}_{\infty, \infty}^{\infty, \infty}$ be a planning instance, and let $c$ be a cost function. The maximum number of times $v \in V$ needs to change value for any optimal solution is

$$
\text { Change }(v) \leq\left|D_{v}\right| \cdot p \cdot\left(\left|D_{v}\right|+1\right)^{p}+\left|D_{v}\right|
$$

where

$$
p=\max \left\{\text { Change }\left(v^{\prime}\right):\left(v, v^{\prime}\right) \in C G(\Pi)\right\}
$$

If there is no variable $v^{\prime}$ then $p=0$.

Proof. In the case where there are not any outgoing variables, then no other variable has any prevailconditions on $v$ and we only have to find the cheapest path to its goal value. The length of the cheapest paths is obviously bounded by $\left|D_{v}\right|$. In this case, $p=0$, which gives us Change $(v) \leq\left|D_{v}\right|$.

In the case where there are outgoing variables, then $p \neq 0$ is the maximum number of times any variable with a prevailcondition on $v$ has to change. It is sufficient to only care about this number because we can always satisfy whatever prevailconditions other variables have on $v$, as long as they change value fewer times. The idea is as follows: since all outgoing variables can only change at most $p$ times then they can only have $p$ prevailconditions on $v$, in some ordering. There are $\left(\left|D_{v}\right|+1\right)^{p}$ unique orders to have prevailconditions on $v$, and for every ordering the variable follows a path in $\operatorname{DTG}(v)$ that visits the values in the ordering. The length of the ordering is $p$ and between any two values the variable has to change value at most $\left|D_{v}\right|$ times. Regardless of how many outgoing variables $v$ has, for every outgoing variable one of the orderings must have satisfied all of its prevailconditions on $v$ for the optimal solution. After having done all this, the variable has to assume its goal value, which it can do in $\left|D_{v}\right|$ variable changes. 
Theorem 7.23. Let $\Pi=(V, A, I, G) \in \overrightarrow{\mathcal{P}}_{k, d}^{\infty, \infty}$ be a planning instance, and let $c$ be a cost function. Then, Change $(v), v \in V$ is bounded by some function, or constant, $\eta=f(k, d)$.

Proof. Without loss of generality, assume that the domain size of every variable size is exactly $k$. Going back to the Theorem 7.22, and more specifically, the value of $p$ : let $O$ be the set of outgoing variables from $v$, then the only possibility for two variables $v_{1}, v_{2}$ to have Change $\left(v_{1}\right)>$ Change $\left(v_{2}\right)$ is if they have different values for $p$ themselves. One way this could happen is if there does not exists a variable that has a prevailcondition on $v_{2}$, but there exists a variable that has a prevailcondition on $v_{1}$. Even if both of them do, at one point during the recursive chain that case must happen, which reflects in their values of $p$. Hence, to determine what child to use to compute the value of $p$, we simply take the variable that has a prevailcondition on $v$ and has the longest chain of children, grandchildren, and so on.

The definition of Change $(v)$ is recursive, but since the longest directed path is bounded, then so is the recursive depth. Furthermore, Change $(v)$ only depend on $k$ and $d$, and both are bounded by a constant, then Change $(v)$ is bounded by some function $f(k, d)$.

To make the complexity analysis of the forthcoming algorithm easier, we make sure that the recurrence relation in the algorithm conforms to the form that is required by the master theorem (Theorem 7.25). The problem with our explanation so far is that there is no obvious upper bound on the number of recursive calls since the degree for every variable in the causal graph is unbounded. One way to solve this is to pass several components to each recursive call. A complication with this is that we need to ensure that the total number of variables to the recursive calls decreases strictly. The algorithm in Figure 7.4 details how to divide, or distribute, the components between the two recursive calls. The DIVIDE function takes a set Components which contains disjunct sets of variables, and the output is two sets $A$ and $B$ such that $A \cup B=C, A \cap B=\varnothing$. The aim is to distribute the disjunct sets into two different sets as evenly as possible (with regard to the total number of variables). The function DIVIDE is a greedy; it starts with $A=\varnothing$, $B=\varnothing$, and for every set adds it to the set that currently contains the fewest variables.

Figure 7.5 presents the algorithm that computes the cost of an optimal solution for instances in $\overrightarrow{\mathcal{P}}_{k, d}^{i, \infty}$. For clarity and conciseness, the algorithm does not construct an optimal solution and instead compute 


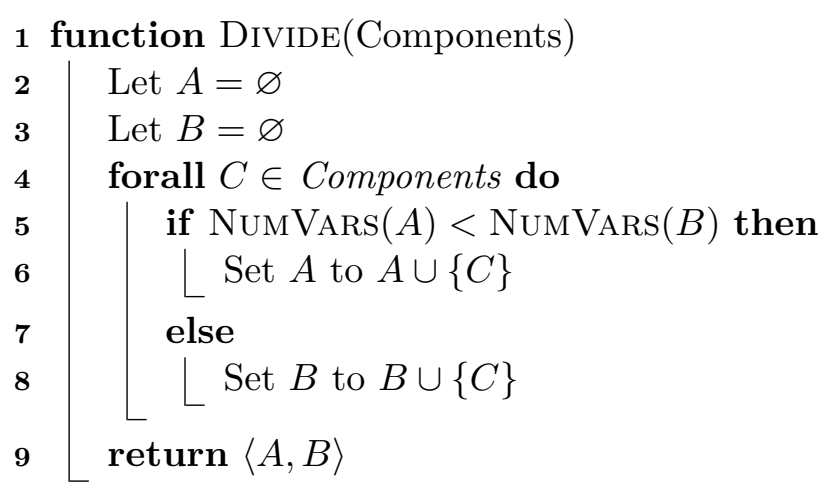

Figure 7.4: A greedy algorithm that distributes components between two sets. The algorithm considers one component at a time, and adds it to the set which has the fewest total number of variables.

the cost of one. In the proof of Theorem 7.21 we had access to a variable $v$ and a sequence of actions $S$ to split with, and we had a solution $\omega$ for $\operatorname{Split}(\Pi, S, v)$. Of course, in practice we do not have access to these. Firstly, we have to select the variable $v$ so that when we split the instance in it then no weakly connected component in the causal graph is too big, and Theorem 7.19 discussed how to do this. Secondly, we also need a sequence of actions $S$ for $v$, which is difficult since the cost of the optimal solution for the splitted instance is affected by the choice of $S$. In an actual implementation, we would most likely need a heuristic function to decide on a good $S$, but for the complexity analysis we simply try all possible sequences $S$ of length up to $\eta$. Since $\eta$ is a constant and the number of actions is bounded by some constant $\kappa=g(k, i)$, where $k, d$ and $i$ are constants, then the number of sequences $S$ that we need to try is bounded by $\sum_{n=0}^{\eta} \kappa^{n}$ (i.e. a constant). Note that, if we do not have a bounded number of actions, then we get an unbounded number of recursive calls. We then split the instance and perform recursive calls, and this is essentially our algorithm. There is one quirk though; if the weakly connected components are too small then we might introduce too many new variables, and to prevent this the base case of the algorithm is to solve weakly connected components of size 4 or less immedatiely by some optimal planner. 


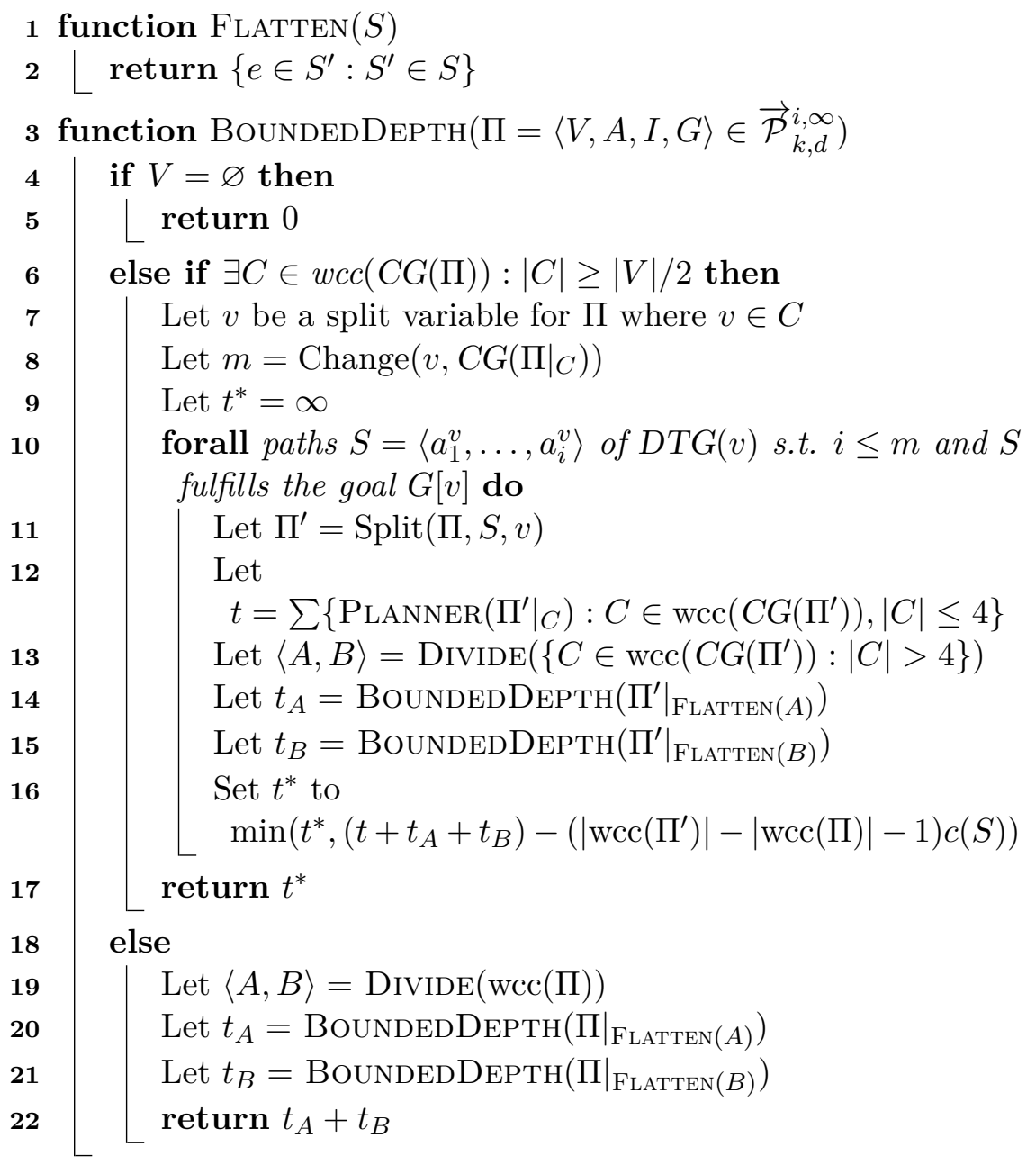

Figure 7.5: Algorithm for computing the total cost of an optimal solution for $\Pi \in \overrightarrow{\mathcal{P}}_{k, d}^{i, \infty}$. 


\subsubsection{Complexity}

Since we introduce variables when we split the instance, we need to make sure that partitioning by the algorithm in Figure 7.4 is sufficiently even.

Theorem 7.24. Let $\Pi$ be a planning instance with variable set $V$. Consider the function call BoundedDePTH(П), which consequently calls Divide. The output of Divide is two sets of components $A$ and $B$ such that $|A| \leq \frac{9}{10}|V|$ and $|B| \leq \frac{9}{10}|V|$.

Proof. There are two calls to Divide in BoundedDepth, and we start with the second one. For this call, there is no component $C$ such that $|C| \geq|V| / 2$. Since every component $C$ is of size $|C|<|V| / 2$, then the difference between $A$ and $B$, with respect to number of variables, is at most $|V| / 2-1<|V| / 2$. Hence, $A$ and $B$ contain at most $\max \{x$ : $\left.2 x-1 \leq \frac{1}{2}\right\}|V|=\frac{3}{4}|V|$ variables, individually. ${ }^{1}$

For the first call, there exists a component $C$ such that $|C| \geq|V| / 2$, and we note that $C$ is the only component of size $|V| / 2$ or greater (otherwise the number of variables would exceed $|V|$ ). Splitting the largest component yields at most $|V| / 5$ components because components of size 4 or less are immediately solved. We also add one variable to every new component, i.e. at most $|V| / 5$ new variables. After splitting, every component has a size between 5 and $|V| / 2$, and from the first case we can conclude that the number of variables in $A$ and $B$ contains is at most $\frac{3}{4}(|V|+|V| / 5)=\frac{9}{10}|V|$ variables, individually.

Before we present the proof for the asymptotic complexity of the algorithm we introduce the master theorem.

Theorem 7.25. Master theorem [CLRS09]. Let $a \geq 1$ and $b>1$ be constants, let $f(n)$ be a function, and let $T(n)$ be defined on the nonnegative integers by the recurrance

$$
T(n)=a T\left(\frac{n}{b}\right)+f(n)
$$

where $\frac{n}{b}$ is interpreted as either $\left\lfloor\frac{n}{b}\right\rfloor$ or $\left\lceil\frac{n}{b}\right\rceil$. Then $T(n)$ has the following asymptotic bounds:

1. If $f(n)=\mathcal{O}\left(n^{c}\right)$ where $c<\log _{b} a$, then $T(n)=\Theta\left(n^{\log _{b} a}\right)$.

\footnotetext{
${ }^{1}$ The largest possible size of a set such that it contains less than $|V| / 2$ more variables than the other set is solved by maximising $x$ in $x-(1-x) \leq \frac{1}{2}$. That is, $x \cdot|V|$ is the largest possible size.
} 
2. If $f(n)=\Theta\left(n^{\log _{b} a}\right)$, then $T(n)=\Theta\left(n^{\log _{b} a} \log n\right)$.

3. If $f(n)=\Omega\left(n^{c}\right)$ where $c>\log _{b} a$ and $a f\left(\frac{n}{b}\right) \leq k f(n)$ for some constant $k<1$ and all sufficiently large $n$, then $T(n)=\Theta(f(n))$.

In the context of a recursive algorithm: $n$ is the size of the problem; $a$ is the number of subproblems in the recursion; $\frac{n}{b}$ is the (maximum) size of each subproblem; and $f(n)$ is extra work done for every recursive call, such as dividing and merging. Our algorithm is designed around the master theorem, and next on the schedule is to apply it.

Theorem 7.26. The asymptotic complexity of the algorithm in Figure 7.5 is $\Theta\left(|V(\Pi)|^{a}\right)$ for instances $\Pi \in \overrightarrow{\mathcal{P}}_{k, d}^{i, \infty}$, where

$$
a=\log _{\frac{10}{9}} 2\left(k(k+1) \sum_{j=0}^{i}\left[k^{j}\left(\begin{array}{l}
i \\
j
\end{array}\right)\right]\right)^{\eta}
$$

Proof. Let $\Pi=(V, A, I, G) \in \overrightarrow{\mathcal{P}}_{k, d}^{i, \infty}$ be a planning instance. Since we are working with polytree causal graphs, then only variables with a degree $>1$ are split variables and the degree of variables introduced by splitting is 1 (i.e. they are never split variables). This means that a split variable will never have a domain size $>k$ (this is not necessarily true for the newly introduced variables). Let $v^{\prime}$ be a split variable of the original instance, and $v$ be a variable introduced by splitting in $v^{\prime}$. Since $\operatorname{DTG}(v)$ is a simple path then it will change value exactly $\left|D_{v}\right|-1$ times, thus $\left|D_{v}\right|-1 \leq \eta$ (for the original instance). Hence, $\eta$ is still a valid upper bound.

Observe that a bound on $|A|$ depends on $|V|, k$ and $i$ : since the causal graph is a polytree, then the actions are unary; every variable's domain size is bounded by $k$, so there are at most $k$ different actions with the same pre- and prevailconditions; and every action can have at most $i$ prevailconditions. Thus the number of actions with an effect on a specific variable is bounded by:

$$
x=k(k+1) \sum_{j=0}^{i}\left[k^{j}\left(\begin{array}{l}
i \\
j
\end{array}\right)\right]
$$

Hence, $|A|$ is bounded by $|V| x$.

Partial states are obviously bounded by a polynomial function that only depends on $|V|$ and $k$, that is, $\|I\|$ and $\|G\|$ are bounded by it. Since the all other components of $\Pi$, that is $\|A\|,\|I\|,\|G\|$, are bounded 
by a polynomial function that takes $|V|, k$, and $i$, then $\|\Pi\|$ is also bounded by some polynomial function that only depends on $|V|, k$ and $i$. Note that $k$ and $i$ are fixed constants. Thus, we can use the master theorem on $|V|$ instead of $\|\Pi\|$.

Theorem 7.23 and Theorem 7.24 also provide an upper bound on the number of recursive calls, and guarantee that the size of the input to them is strictly smaller. More specifically, since the selected variable $v$ can change at most $\eta$ times, and there are at most $x$ actions that has an effect on $v$, then the number of action sequences is bounded by $x^{\eta}$. For every such sequence we make two recursive calls, which gives us a total of $2 x^{\eta}$ recursive calls.

The master theorem requires the recurrence to be of the form $T(n)=a T\left(\frac{n}{b}\right)+f(n)$, which we have.

- $n=|V|$ (size of the problem),

- $a=2 x^{\eta}$ (number of recursive calls),

- $b=\left(\frac{9}{10}\right)^{-1}=\frac{10}{9}$ (size of subproblems, i.e. Theorem 7.24), and

- $f(n)=\mathcal{O}\left(n^{3}\right)$.

Case (1) of the master theorem states that if $f(n)=\mathcal{O}\left(n^{c}\right)$ where $c<\log _{b} a$, then $T(n)=\Theta\left(n^{\log _{b} a}\right)$. The lowest sensible values for $k$ and $i$ are 2 and $0(d=0$ implicitly), respectively, which implies $a \geq 2 \cdot(2 \cdot 3)^{2}=72$. Since $3<\log _{\frac{10}{9}} 72 \approx 40.59$, then the asymptotic complexity of the algorithm is $\Theta\left(|V|^{\log _{\frac{10}{9}} a}\right)$.

A brief note on $f(n)$ in the proof. We have not shown that the work outside of the recursive calls, that is, finding a split variable, splitting the instance, and so on, can be done in $\mathcal{O}\left(n^{3}\right)$. It is, however, obvious that this can be done in polynomial time with a reasonable exponent, moreover, we have leeway for the exponent to be as large as 40 .

\subsubsection{Soundness, Completeness \& Optimality}

We now show soundness, completeness and optimality of the algorithm in Figure 7.5. The proofs are straightfoward and rely heavily on Theorem 7.21 and 7.22 .

Theorem 7.27. Soundness.

Let $\Pi \in \overrightarrow{\mathcal{P}}_{k, d}^{\infty, \infty}$, and $c=\operatorname{BoundedDePth}(\Pi)$. If $c \neq \infty$, then there exists a solution for $\Pi$ with $\operatorname{cost} c$. 
Proof. Let $\Pi=(V, A, I, G) \in \overrightarrow{\mathcal{P}}_{k, d}^{\infty, \infty}$. There are three cases in the algorithm: (1) $V=\varnothing ;(2)$ there exists a component $C \geq|V| / 2 ;(3)$ otherwise.

(1) Returns 0 since the goal is met in the initial state, i.e. $G(\Pi) \subseteq$ $I(\Pi)$. This case only happens if the original instance does not have any variable.

(2) We have a split variable $v$, a sequence of actions $S$ for $v$, and $\Pi^{\prime}=\operatorname{Split}(\Pi, S, v)$. By construction (Definition 7.20), we can solve the projections induced by the weakly connected components $C_{1}, \ldots, C_{j}$ in $C G\left(\Pi^{\prime}\right)$ independently. According to Theorem 7.21 the total cost of an optimal solution for $\Pi^{\prime}$ is $\sum_{i=1}^{j} c_{i}-|\operatorname{wcc}(\operatorname{Split}(\Pi, S, v)) \backslash \operatorname{wcc}(\Pi)| c(S)$, where $c_{i}$ is the cost of an optimal solution for $\left.\Pi^{\prime}\right|_{C_{i}}$.

(3) It is obvious that several projections induced by weakly connected components in the causal graph can be (optimally) solved independently and that their solutions can be interleaved in any way to construct an optimal solution.

\section{Theorem 7.28. Completeness.}

Let $\Pi \in \overrightarrow{\mathcal{P}}_{k, d}^{\infty, \infty}$, and $c=\operatorname{BoundedDepth}(\Pi)$. Then $c=\infty$ if and only if $\Pi$ is unsolvable.

Proof. Let $\Pi=(V, A, I, G) \in \overrightarrow{\mathcal{P}}_{k, d}^{\infty, \infty}$. Suppose $c=\infty$ and that there exists a solution $\omega$ for $\Pi$. We note that the algorithm has three cases: (1) $V=\varnothing$; (2) there exists a component $C \geq|V| / 2$; (3) otherwise. One of these cases must yield $\infty$ despite the existence of a solution.

(1) Returns 0 since the goal is met in the initial state, i.e. $G(\Pi) \subseteq$ $I(\Pi)$. This contradicts the assumption.

(2) Theorem 7.21 says that there exists a sequence of actions $S$ for every $v$ such that the instance $\operatorname{Split}(\Pi, S, v)$ is solvable if and only if $\Pi$ is. Theorem 7.22 states that $|S| \leq \eta$ for some optimal solution, and the algorithm enumerates all such sequences. Therefore the sequence will eventually be found and $\infty$ will not be returned. This contradicts the assumption.

(3) This case cannot yield $\infty$ since we can solve the projections induced by weakly connected components in the causal graphs independently, and interleave their solutions in any way. We sum their costs and no cost is $\infty$ unless the input instance is unsolvable. This contradicts the assumption. 


\section{Theorem 7.29. Optimality.}

Let $\Pi \in \overrightarrow{\mathcal{P}}_{k, d}^{\infty, \infty}$, and $c=\operatorname{BoundedDePth}(\Pi)$. Then, there does not exists a solution for $\Pi$ with $\operatorname{cost} c^{\prime}$ such that $c^{\prime}<c$.

Proof. Since the algorithm is complete (Theorem 7.28), we assume that $\Pi$ is solvable. In other words, there exists an optimal solution for $\Pi$ with cost $c^{\prime}$. Let $c=\operatorname{BoundedDePth}(\Pi)$, and assume $c>c^{\prime}$. Then suboptimality must have been introduced in at least one of the three cases: (1) $V=\varnothing ;$ (2) there exists a component $C \geq|V| / 2$; (3) otherwise.

(1) The algorithm always returns 0 , which is the optimal cost for instances with no variables. In other words, suboptimality cannot have been introduced in this case.

(2) Theorem 7.21 and 7.22 says that there is no longer sequence $S$ which would yield a split with a smaller cost for $\Pi$ (i.e. after compensating for the additional costs). Since there exists a solution for $\Pi$ with cost $c^{\prime}$ then a sequence $S,|S| \leq \eta$ can be extracted from it. Furthermore, the algorithm enumerates every sequence $S^{\prime},\left|S^{\prime}\right| \leq \eta$, computes the cost of $\operatorname{Split}(\Pi, S, v)$ and stores the cost of the cheapest split. However, since $c>c^{\prime}$ it must never have encountered $S$ - a contradiction.

(3) Since we can solve projections induced by weakly connected components in the causal graph independently, and optimally, to construct an optimal solution (or cost) for the original instance. Hence, suboptimality cannot have been introduced in this case.

\subsubsection{Discussion}

At the end of the proof for Theorem 7.26, we considered the lowest sensible values for the fixed constants $k, i$ and $d$, and derived an unflattering exponent $\geq 40$. This is probably a very pessimistic upper bound since it depends on Change is likely not a tight estimate. If the goal is to find an optimal solution, then the algorithm must try all possible action sequences to split with. However, if an suboptimal solution is acceptable then it can stop after finding a split that has a solution. However, this does not change the complexity analysis, but it might perform well in practice. Furthermore, there are plenty of existing heuristic functions that could suggest action sequences to split with. Consider a solution $\omega$ for a variable projection that contains the split variable. We can then extract an action sequence for a split variable from $\omega$ by removing actions that do not have an effect on the split variable. Of course, for every remaining action we need to find 
an action in the original instance that projects to it. In other words, the co-operation of some abstraction method, or heuristic function, and this algorithm could prove worthwhile to solve polytree instances.

Polytree causal graphs might not occur frequently in practice, although this does not mean that they are not useful. It is possible to transform causal graphs to polytrees by removing pre-, prevailconditions and effects, but this also means that solutions are no longer valid for the original instance. However, such solutions can still be used to form a distance heuristic for the original instance. This strategy is also performed by another causal graph heuristic [Hel04].

Another observation is that many parts of the algorithm can be used for other causal graph structures. A vertex in an undirected connected graph is an articulation point (or cut vertex) if and only if removing it disconnects the graph. In order words, such vertices in a causal graph are split variables, even if the causal graph is not a polytree. It might be rewarding to split the instance and use other means to solve projections induced by the weakly connected components in the causal graph. After all, the state transition graph grows exponentially with the number of variables, so if one is fortuitous and splits the variable with a good action sequence then the reward would be substantial. 



\section{Chapter Eight Variable Similarity}

The last topic in this thesis is variable similarity. This topic might be a bit controversial since there is no similar research, and because we analyse unsolvable projections with a very pragmatic approach. In Chapter 7 we exploited the fact that when two variables are isomorphic (i.e. extremely 'similar'), then we can aggregate them into one. This is not uncommon; many theoretical results exploit similarities to deduce when we can do something without ruining some purpose. For example, in M\&S it is favourable to aggregate states with similar distances (or costs) to the initial state and the goal states, and the purpose is to minimise the size of the abstract state space without sacrificing too much information. Furthermore, when two states meet specific conditions states, they can be aggregated without affecting solvability with respect to subsequent merges. In some sense, the set of conditions denotes when two states are sufficiently similar to be exploited.

In this chapter we look into a much more general notion of similarity which might be useful despite the fact that we have no theoretical understanding. The idea is to capture the cases above and other ones. Of course, the tool is not perfect but might function well in practice. More specifically, we devise a variable similarity measure that rates how similar two variables are on a scale (between 0 and 1 ). This measure takes into account the domain transition graph of both variables and the causal graph. Regarding the latter, we consider direct and indirect dependencies, and whether the indirect dependencies are also similar. In other words, two variables are similar if they have similar domain transition graphs and if they have dependencies on similar variables. Consider an instance from some logistics domain; two trucks should be considered to be similar if they are able to visit the same areas and pick up the same packages. This is symbolised by having identical, or similar, domain transition graphs, and if they have arcs to the same 
variables in the causal graph then they, presumably, have actions to load and unload the same packages. We use the measure to analyse unsolvable variable projections and observe that they tend to consist of dissimilar variables, more so than solvable projections.

\subsection{Definitions}

As we mentioned briefly earlier, the variable similarity measure takes into account: (1) how similar the two domain transition graphs are and (2) how similar the adjacent variables in the causal graph are. In order for two variables to be considered similar, they must depend on similar variables. The latter part is defined as a modification of SimRank [JW02], which is a general similarity measure. SimRank computes a similarity value for any domain with object-to-object relationships. Jeh and Widom [JW02] explain that SimRank, intuitively, considers two objects to be similar if they are referenced by similar objects, which coincides with our earlier discussion. Furthermore, many instances encoded with the $\mathrm{SAS}^{+}$formalism also capture the notion of objects and relationships: the variables represent the objects, and the actions represent the relationships. The input to SimRank is a graph, and in our case, an abstract overview of the instance and its dependencies is provided by the causal graph. Our equations differ slightly from what Jeh and Widom [JW02] gave. We use their minimax variant, but we let $s\left(v_{1}, v_{2}\right)$ take the average rather than the minimum in order to not ignore either the in- or outgoing edges completely. The equations we use are:

$$
\begin{gathered}
s_{\text {IN }}\left(v_{1}, v_{2}\right)=\frac{C \cdot \sum_{v_{1}^{\prime} \in \operatorname{IN}\left(v_{1}\right)} \max \left\{s_{\text {IN }}\left(v_{1}^{\prime}, v_{2}^{\prime}\right): v_{2}^{\prime} \in \operatorname{IN}\left(v_{2}\right)\right\}}{\max \left(\left|\operatorname{IN}\left(v_{1}\right)\right|,\left|\operatorname{IN}\left(v_{2}\right)\right|\right)} \\
s_{\text {OUT }}\left(v_{1}, v_{2}\right)=\frac{C \cdot \sum_{v_{1}^{\prime} \in \operatorname{OUT}\left(v_{1}\right)} \max \left\{s_{\text {OUT }}\left(v_{1}^{\prime}, v_{2}^{\prime}\right): v_{2}^{\prime} \in \operatorname{OUT}\left(v_{2}\right)\right\}}{\max \left(\left|\operatorname{OUT}\left(v_{1}\right)\right|,\left|\mathrm{OUT}\left(v_{2}\right)\right|\right)} \\
s\left(v_{1}, v_{2}\right)=\frac{s_{\mathrm{IN}}\left(v_{1}, v_{2}\right)+s_{\text {OUT }}\left(v_{1}, v_{2}\right)}{2}
\end{gathered}
$$

Where $\operatorname{IN}\left(v_{1}\right)=\left\{v_{2}:\left(v_{2}, v_{1}\right) \in C G(\Pi)\right\}$ and $\operatorname{Out}\left(v_{1}\right)=\left\{v_{2}:\right.$ $\left.\left(v_{1}, v_{2}\right) \in C G(\Pi)\right\}$, i.e., the neighbours that $v$ is connected to with an in- and outgoing arc, respectively. If either IN or OUT returns an empty set, then $s_{\text {IN }}\left(v_{1}, v_{2}\right)=0$ and $s_{\text {OUT }}\left(v_{1}, v_{2}\right)=0$. Intuitively, how similar a variable $v_{1}$ is to a variable $v_{2}$ depend on if every adjacent variable of $v_{1}$ (in the causal graph) can be matched with a similar neighbour of 
$v_{2}$, which is done recursively. We refer the reader to Jeh and Widom for how to efficiently compute the value. The constant $C$ is what Jeh and Widom called the decay factor, and its purpose is to let the closest relationships have the most impact on the final similarity rating. We replace $C$ with a function $d\left(v_{1}, v_{2}\right)$ which allows us to influence the final similarity rating with regard to how similar the DTGs of the two variables $v_{1}$ and $v_{2}$ are.

A natural choice of computing a similarity rating of two DTGs is to compute the graph edit distance and divide it with some upper-bound of the edit distance. The graph edit distance is the smallest number of operations required to transform a graph to another, by using operations such as vertex insertion, vertex deletion, edge insertion, edge deletion, and so on. A naive upper-bound is to delete every vertex and edge, and then insert every vertex and edge in the goal graph. However, computing the graph edit distance is NP-hard $\left[\mathrm{ZTW}^{+} 09\right]$. Instead, the similarity rating $d\left(v_{1}, v_{2}\right)$ is computed in the following way:

1. For every pair of vertices $d_{1}, d_{2}$ in $\operatorname{DTG}\left(v_{i}\right)$, compute the cheapest cost $c$ from $d_{1}$ to $d_{2}$, and insert $c$ into a sorted list $l_{i}$;

2. Compute the string edit distance (polynomial time) $s$ for transforming $l_{1}$ to $l_{2}$ (symbol insertion, deletion and substitution); and

3. Return $1-\left(s / \max \left\{\left|l_{1}\right|,\left|l_{2}\right|\right\}\right)$.

This method of computing a graph similarity rating is simple, but given two variables $v_{1}, v_{2}$ with isomorphic DTGs then $d\left(v_{1}, v_{2}\right)=1$ (i.e., $s=0)$.

We extend the function $s$ to sets of variables, and take the pairwise average:

$$
s(V)=\frac{1}{\left(\begin{array}{c}
|V| \\
2
\end{array}\right)} \sum_{v_{i}, v_{j} \in V, i<j} s\left(v_{i}, v_{j}\right)
$$

We take the pairwise average since, as discussed above, we want a good mix between variables that are depended on and depends on other variables. Taking, for example, the maximum, could be bad since there might be good combinations with two similar variables. The next problem is that $s(V)$ does not guarantee that the range of $s(V)$ is between 0 and 1 , or that they are evenly distributed. Hence, to give a domain-independent threshold we need to normalise and adjust the ratings produced by $s$. A straightforward way is to find the range of $s$, 
take the minimum $\min$ and the maximum $\max$ of it, and then adjust the output:

$$
s^{*}(V)=\frac{s(V)-\min }{\max -\min }
$$

In the upcoming statistical analysis, the variable projection with the most similar variables is rated 1 , and the projection with the least similar variables is rated 0 .

\subsection{Statistical Analysis}

In this statistical analysis we consider projections at the lowest inconsistent level, i.e. there are no unsolvable projections with strictly fewer variables. The objective of this analysis is to find out whether the 'core' of unsolvable projections tend to consist of dissimilar variables. The risk of considering larger projections is that the core can be obscured by all other variables. Furthermore, as we noted in Section 6.5.3, PDBs of unsolvable projections tend to contain many dead-end entries, thus this analysis is relevant for PDBs too.

Figure 8.1 contains histograms of the similarity ratings for both unsolvable projections, and all projections, for the lowest inconsistent level $k$. For most domains, the similarity measure partitions unsolvable and solvable projections surprisingly well; unsolvable projections tend to have a very low similarity rating (close to $0 \%$ ). This is very good news since we can exploit this by checking projections with a low similarity rating. For 3-SAT and Pegsol, unsolvable projections tend to have a lower similarity rating, however, the contrast is not as extreme. Every projection in the domain Tiles had the same similarity rating, thus the measure was unable to differentiate between them. For level $k+1$ (Figure 8.2) the differences are not as distinct. One explanation is that almost all of them are successors of some unsolvable projection, and the added (superfluous) variable is very often similar to the variables of the unsolvable predecessor(s).

\subsection{Improving Consistency Checking}

One obvious way to utilise the variable similarity measure is to filter out solvable projections for consistency checking. A disadvantage of doing this is that we can no longer guarantee $k$-consistency since we might filter out every unsolvable projection. If this happens then inconsistency 

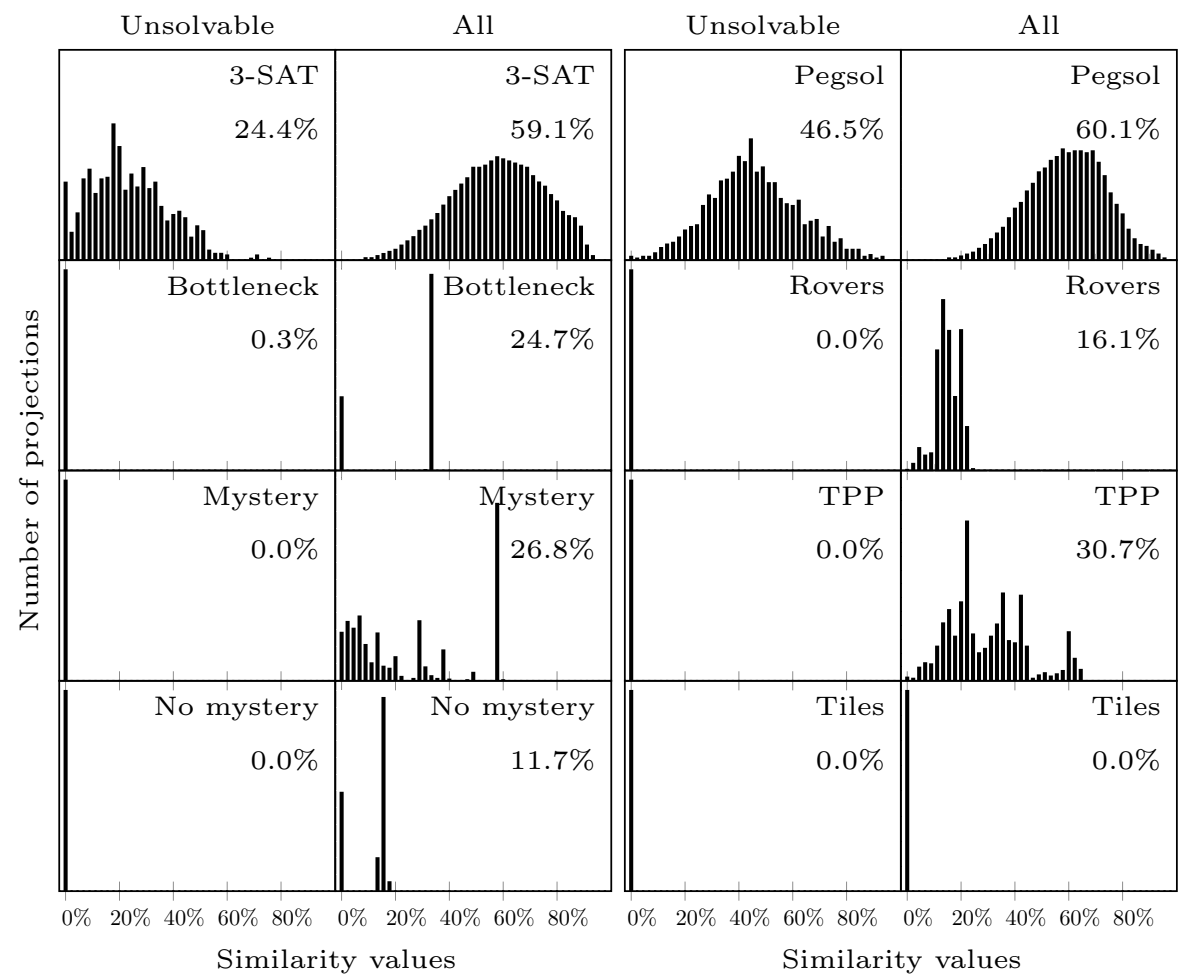

Figure 8.1: Histograms for every domain over the similarity rating of projections for the lowest inconsistent level $k$. The histograms to the left are restricted to unsolvable projections, and the corresponding histograms to the right are not restricted. 

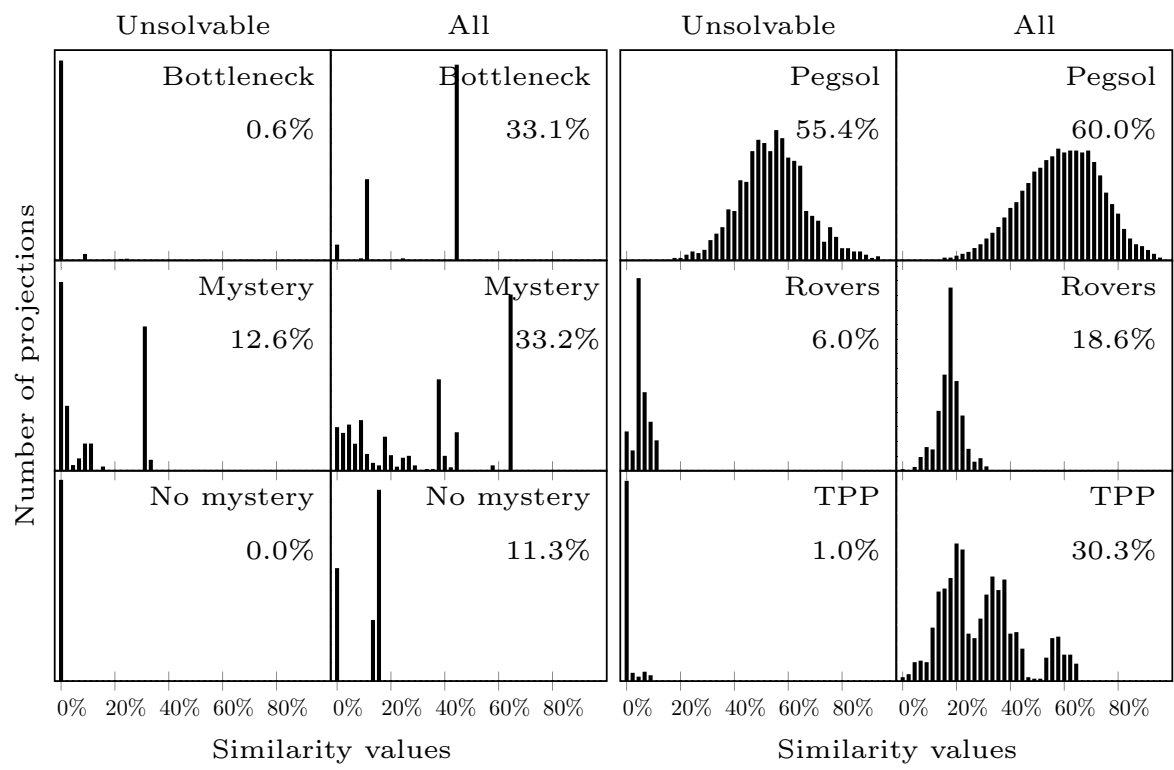

Figure 8.2: Histograms for every domain over the similarity rating of projections for one level above the lowest inconsistent level $k$, i.e. $k+1$. The histograms to the left are restricted to unsolvable projections, and the corresponding histograms to the right are not restricted. 
must instead be found for a $k^{\prime}>k$. Based on the statistical analysis, this scenario seems unlikely.

In addition to the variable similarity measure, we also exploit observations made in the statistical analysis for consistency checking, i.e. Section 5.3. Suppose we know the percentage $p$ of unsolvable projections for level $k$, and let $u$ be the number of unsolvable projections that we aim to not filter out. Then we use the following conditions to decide whether to check a projection $\left.\Pi\right|_{P}$ for some instance $\Pi$ :

1. if $G(\Pi)$ is defined for some variable in $P$,

2. if $C G\left(\left.\Pi\right|_{P}\right)$ is a weakly connected component,

3. if we have not checked $\frac{u}{p}$ projections,

4. if the similarity rating of $P$ is below the median rating for level $k$ (sort w.r.t. the rating, and only consider the most dissimilar half), and

5. if $r \geq(1-c)^{1 / u}$, where $r \in[0,1]$ is a random number from an uniform distribution, and $c \in[0,1]$ influences the probability that the current level is consistent.

Conditions (1) and (2) were used in the first version of consistency checking, and are repeated to serve as a reminder. The statistical analysis in Section 5.3 revealed how many unsolvable projections there are, even though they are scarce, they are not extremely rare. Furthermore, they tend to be less scarce at higher levels. Condition (3) exploits this by generating a subset of level $k$, which contains $\frac{u}{p}$ random projections with $k$ variables; and the expected number of unsolvable projections in this set is $u$. For example, if $u=10$ and $p=0.0001$ then we generate a set of 100000 random projections of size $k$. According to the statistical analysis, if there is not a single unsolvable projection in this set, then it is very likely that $\Pi$ is $k$-consistent. Condition (4) is fairly straightfoward: we ignore projections with similar variables. If every projection has an unique rating, then we filter out about half the projections (which might seem cautious). Continuing on the previous example, we filter out about 50000 of the randomly generated projections that we have reason to believe are solvable. Condition (5) exploits the fact that we only need to find 1 unsolvable projection, and that there often are several unsolvable projections. Suppose there are 10 unsolvable projections and we give every projection a $30 \%$ chance of being checked, then the probability of not checking any of the 10 unsolvable projections is $(1-0.3)^{10} \approx 2.8 \%$. 
In other words, $70 \%$ of all projections are filtered out, yet there is a $97.2 \%$ chance that there still is an unsolvable projection to check. In the previous example, the final number of projections to check is about 15000. Note that, in condition (5), we give the probability of filtering out every unsolvable projection.

Condition (3) and (5) are approximately the same, both filter out projections blindly based on the given probabilities. The main difference is that condition (3) establishes a set of projections for other conditions to operate on. The implementation does not does not take every projection into account, it performs a breadth-first search with a bounded branching factor (branches are selected randomly). This hopefully avoids the potential problem of bias with respect to how frequently variables are selected. Furthermore, since we know that unsolvable projections do tend to cluster up, we want an 'uneven' search to increase the likelyhood of finding a cluster. Recall that the pattern database method $H C_{D E}$ had a tendency of finding the same cluster several times because it selected similar branches in its search. Additionally, this implementation is often necessary since representing every projection of size $k$ can make the method run out of memory, or time from generating them.

The variable similarity measure cannot improve our pattern database methods in any significant way since they already select amongst the best patterns within the given restrictions for dead-end detection. The measure could be useful for guiding the initial part of the hill-climbing search since small PDBs might not contain any dead-end, thus the successor is selected arbitrarily. When this happens, we can use the measure as a fall back, and select a successor of dissimilar variables.

\subsection{Experimental Evaluation}

In this section, we evaluate different configurations of consistency checking on all benchmarks. We gave every configuration 30 minutes and $4 \mathrm{~GB}$ of available memory for every instance. Every configuration uses a subset of the filters presented in Section 8.3, and the constants are: $c=90 \%, p=0.01 \%, u=10$. Since the frequency of unsolvable projections tends to increase for higher levels, we do not aim to find inconsistency at the lowest possible level, but rather one above.

The coverage results are detailed in Table 8.1. Note that configuration $\{1,2\}$ is the configuration from Chapter 5 . The figures within parentheses are the average level inconsistencies were found at for the in- 


\begin{tabular}{lrrrrr}
\hline Domain $(\#)$ & $\{1,2\}$ & $\{1,2,3\}$ & $\{1,2,3,4\}$ & $\{1,2,3,4,5\}$ & $\{1,2,3,5\}$ \\
\hline 3-SAT (30) & $0(-)$ & $1(18.0)$ & $5(18.8)$ & $5(17.8)$ & $5(18.0)$ \\
Bottleneck (25) & $20(1.0)$ & $20(1.0)$ & $20(1.0)$ & $20(1.0)$ & $20(1.0)$ \\
Mystery (9) & $9(1.0)$ & $9(1.0)$ & $9(1.0)$ & $9(1.0)$ & $9(1.0)$ \\
No mystery (25) & $25(3.6)$ & $25(3.6)$ & $25(3.6)$ & $25(3.7)$ & $25(3.7)$ \\
Pegsol (24) & $6(1.0)$ & $7(3.6)$ & $15(13.1)$ & $24(16.5)$ & $24(16.5)$ \\
Rovers (25) & $9(1.4)$ & $9(1.9)$ & $10(2.4)$ & $11(4.2)$ & $12(3.9)$ \\
Tiles (20) & $10(8.0)$ & $10(8.0)$ & $10(8.0)$ & $10(8.0)$ & $10(8.0)$ \\
TPP (25) & $6(1.0)$ & $6(1.0)$ & $7(1.7)$ & $9(4.1)$ & $7(2.0)$ \\
\hline Total (183) & 85 & 87 & 101 & 113 & 112 \\
Total* $(109)$ & 69 & 69 & 71 & 74 & 73 \\
\hline
\end{tabular}

Table 8.1: Coverage results when using different filters. The figures in the set denote which filters are used from the list in Section 8.3, and the figures within parentheses are the average level inconsistencies were found at. Every configuration used mutexes generated by $h^{3}$. The second total excludes 3-SAT, Pegsol and Tiles.

stances they showed unsolvability, i.e. the average might differ between configurations that show unsolvability for different instances. Perhaps the most surprising configuration is $\{1,2,3\}$; restricting consistency checking to check a fixed number of projections per level did not give a sigificant increase in coverage. One would assume that giving up early on a level that has proven to either contain no or very few unsolvable projections would be helpful, but 100000 projections per level might still be too many. For Pegsol instances, this configuration reached level 16 (out of 33 levels), whilst $\{1,2\}$ reached level 8 , before running out of time. However, this improvement was obviously not enough to find an unsolvable projection.

The next configuration, $\{1,2,3,4\}$, performed a lot better. Most notably, it proved an additional 8 Pegsol and 4 3-SAT instances as unsolvable. Regarding the first domain, this configuration found unsolvable projections around level 20, but could not go any further since it ran out of time. The number of projections it checked per level was around 50000 .

Out of these configurations, the best performing one was $\{1,2,3,4,5\}$ (i.e. every filter was used); the increase in coverage came mostly from Pegsol, and some from 3-SAT, Rovers and TPP. This configuration found unsolvable projections between level 20 and 24 for most Pegsol instances, and it checked around 10300 projections per level. When 


\begin{tabular}{lrrr}
\hline Domain $(\#)$ & $\{1,2,3\}$ & $\{1,2,3,4\}$ & $\{1,2,3,4\}+h^{3}$ \\
\hline 3-SAT (30) & $10(37.1)$ & $10(34.2)$ & $10(33.8)$ \\
Bottleneck (25) & $12(4.3)$ & $18(5.5)$ & $20(1.0)$ \\
Mystery (9) & $7(4.1)$ & $4(4.3)$ & $9(1.0)$ \\
No mystery (25) & $24(5.1)$ & $25(4.7)$ & $25(3.6)$ \\
Pegsol (24) & $20(26.3)$ & $21(25.5)$ & $21(18.6)$ \\
Rovers (25) & $5(9.8)$ & $5(11.8)$ & $9(2.7)$ \\
Tiles (20) & $10(9.0)$ & $10(9.0)$ & $10(8.0)$ \\
TPP (25) & $4(14.5)$ & $11(15.5)$ & $11(10.2)$ \\
\hline Total (183) & 92 & 104 & 115 \\
Total* $(109)$ & 52 & 63 & 74 \\
\hline
\end{tabular}

Table 8.2: Coverage results for three configurations. The figures in the set denote which filters are used from the list in Section 8.3, and the figures within parentheses are the average level inconsistencies were found at. The third filter reduces to 100 and 100000 projections for $\{1,2,3\}$ and $\{1,2,3,4\}$, respectively, and the fourth filter reduces to 100 projections. No configuration used mutexes unless stated otherwise. The second total excludes 3-SAT, Pegsol and Tiles.

filter 4 was removed from the best configuration, i.e. $\{1,2,3,5\}$, then some coverage was lost for TPP since it ran out of time, but it gained some coverage in Rovers.

Consider the increase in the average level inconsistencies were found at for Pegsol in Table 8.1. This increase does not stem from the methods skipping many smaller unsolvable projections, but rather that the lowest inconsistent level was too high for some configurations. We also note a similar result for Rovers. Of course, the methods can fail to find an unsolvable projection at the lowest inconsistent level, but it is rare that it does not find an unsolvable projection at the lowest inconsistent level or the next level.

It is not obvious from Table 8.1 how useful the variable similarity measure is. Hence, in Table 8.2 we test two configurations and both are limited to 100 projections. The configurations are $\{1,2,3\}$ and $\{1,2,3,4\}$, where the third filter reduces to 100 and 100000 projections for respective configuration. The fourth filter, instead of halving, ensures that the 100 most dissimilar projections are left to be checked. We also included one configuration that uses mutexes so that we can compare the figures with the ones in Table 8.1. The configuration with the 
similarity filter performs overall better (104) than the configuration without it (92). The similarity filter works well on Bottleneck and TPP instances, but has an opposite effect on Mystery instances. The histograms for Mystery show that there are a fair amount of solvable instances that are also very dissimilar, but this does not explain why configuration $\{1,2,3\}$ performs well on Mystery instances.

\subsection{Discussion}

Limiting the number of projections to check for every level appears to be crucial for good performance. The statistical analysis throughout this thesis supports this since the frequency of unsolvable projections is not extremely low for the inspected instances. By exploiting this we increased the total coverage from 85 to 112 - 115, depending on configuration. In Section 6.5, the method that identified the most initial states as dead-ends was Mrg1 with 114, or Aidos with 94 for the second total, and one of the configurations barely beat Mrg1. Consistency checking can be improved further by correctly identifying how many projections to consider per level, after all, we could detect 5 more 3-SAT instances by limiting to 100 projections per level.

Even though the variable similarity measure did not have an important impact on the total coverage, it safely removed most solvable projections and thus improved the running time significantly. Since the measure is very fast to evaluate and it removed half of the projections to check per level, then it effectively halved the running time for sufficiently large instances. Nevertheless, it might be the case that the variable similarity measure can be exploited in different and better ways. Perhaps, by refining the variable measure we could provide a more distinct partitioning between unsolvable and solvable projection. For example, we only take into account preconditions and effects indirectly via the causal graph, and by considering them directly we might end up with a better measure. It is important to note that the variable measure never backfired. In the worst observed case, Tiles, it simply gave the same similarity to every projection. In other words, it degraded elegantly: it gave no information, rather than misleading information. Of course, this may not be the case for every domain and has to be investigated further. 



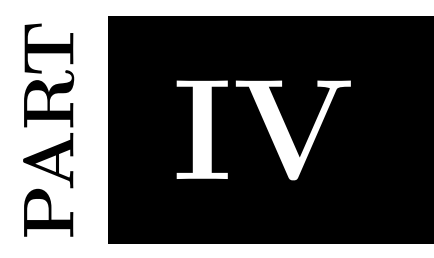

Concluding Remarks 



\section{Chapter Nine \\ Concluding Remarks}

This thesis is about to come to a conclusion, and it is time to summarise what we have learned. The theme of this thesis is unsolvable planning instances - a facet of planning that has historically been neglected - and we devised several methods for detecting unsolvable planning instances using variable projection. Furthermore, we scrutinised variable projections statistically to outline various properties of projections, which are exploited in a range of ways.

The first method was consistency checking, which checks every projection with a certain number of variables. The method starts with projections with $i=1$ variables, and if all such projections are solvable then $i$ is incremented by 1 . This is repeated until either an unsolvable projection is found, or $i$ is larger than some fixed constant $k$. As we noted in Chapter 8 , it is essential to reduce the number of projections to check since there are simply too many of them. Suppose we want to check every projection with 10 variables of an instance with 30 variables, then (if the undirected causal graph is a complete graph) we have to check about 30 million projections - an infeasible task. If unsolvable projections are not exceedingly rare then after checking 1 million projections, it becomes questionable to check any more projections. The statistical analysis revealed that even though unsolvable projections are uncommon at the lowest inconsistent level, there are still sufficiently many of them for proving unsolvability. We exploited this by limiting the number of projections to consider per level, and it had an immense impact on the performance of the method.

A flaw of consistency checking is that if we never find an unsolvable projection, then all of the computations are wasted. The second method, pattern databases, mends this by storing every encountered dead-end state in a data structure, which is then used to prune the state transition graph of the original instance. Of course, this could be added 
to consistency checking but then we have to use a regression search algorithm and store them at the expense of time and space. However, it is often worthwhile to pay this price in practice. In Chapter 6 we devised an opportunistic method which do what we just discussed. By an extensive statistical analysis, we showed that this method found very good PDBs for the purpose of dead-end detection. We also showed that PDBs of unsolvable projections tend to contain a lot of dead-end entries, which explained why the method also found many unsolvable projections. Out of all of our proposed methods, this method had the best performance with respect to total coverage.

We also explored polytree causal graphs, and unearthed some tractable classes. The techniques used to find these classes gave inspiration to a variable similarity measure. This measure was able to distinguish between unsolvable and solvable projections fairly well, and was used to optimise the running time of consistency checking.

\section{Future Research Directions}

We have already discussed a few research directions, and we extend on some of these and also discuss some additional ones.

\section{Refining Variable Projection}

Variable projection is a fairly coarse abstraction method; every variable that we add to a projection, at least doubles the size of its state transition graph. We demonstrated that variable projection can be refined with the help of mutexes, which could turn previously solvable projections into unsolvable ones. They could also increase the number of dead-ends in a projection. However, there might be other techniques that are also useful for variable projection.

Since we are only interested in (un)solvability, then it might be possible to simplify an instance such that the instance is solvable if and only if the simplified instance is. Hopefully, it is easier in practice to prove that the simplified instance is unsolvable. One such simplification is to remove redundant actions [HJ00], which are actions that can be replaced by a sequence of other actions. In other words, if a redundant action is used in some solution, then we can construct another solution without the redundant action. Thus, we can remove the redundant action without affecting solvability. With respect to some projection, if we remove a redundant action then we potentially remove a transition in 
its state transition graph. In the original instance, this does not matter as much since, per definition, there is another path to the target state but this is not necessarily true for the projection. For example, if the redundant action has preconditions on a different set of variables than the sequence of actions it is redundant to, and the variable projection was not projected onto some of those variables, then the redundant action can be applicable in states where the sequence of actions is not. Therefore, removing redundant actions might be helpful.

\section{Refining the Variable Similarity Measure}

The statistical analysis of the variable similarity measure is compelling: the similarity values of unsolvable projections were much closer to 0 than the similarity values of all projections. In many domains, every unsolvable projection got 0 as a score, or very close to it. However, the statistical analysis is restricted to those instances where we could solve every projection at the lowest inconsistent level. Furthermore, since there is little formal understanding of the variable similarity measure, it is difficult to get an understanding of the generality of the measure. A more extensive analysis must be performed to see if the result holds, and if it does not then it can still useful: we can design a heuristic function that decides when it should be used. We suspect that the variable similarity measure works well on 'natural' domains, e.g. logistics, but less so on domains such as 3-SAT since it is not clear what every variable in the planning instance represents.

The variable similarity measure can be improved by taking into account more information. For instance, at the moment it only considers preconditions and effects indirectly via the causal graph. Furthermore, the way that we compute the similarity between two domain transition graphs is very crude, and there are many other ways of measuring graph similarity. Hopefully, by improving these aspect of the measure, it can then partition between unsolvable and solvable projection more accurately.

\section{Consistency Checking}

Two approaches of improving consistency checking is by refining variable projection - which we have discussed - and by improving the filters, which decides whether a projection should be checked. It is critical to reduce the number of projections to check and to do it in an 'informative' fashion. At the moment, the informative filters that we have are: (1) 
check if the projection has a goal variable; (2) check if causal graph of the projection is weakly connected; (3) check if the variable similarity value of the projection is sufficiently low. The first two are very simple, and the third one is somewhat difficult to exploit in its current state. Hopefully, by refining the variable similarity measure, we can set a much more aggressive threshold and improve the performance of consistency checking even more.

\section{Pattern Databases}

The scoring function of $H C_{D E}$ counts the number of dead-ends, and when $H C_{D E}$ is in the scenario where the PDB of every successor contains no dead-ends, then it has to make an uninformative decision of which one to choose as the successor. This is obviously bad, and one approach to decide tie-breaks is to use the variable similarity measure. Since the measure considers structural information encoded in the causal graph and the domain transition graphs, then it can compute different scores for the successors. However, it is not clear whether this would lead to any improvement since $H C_{D E}$ already constructs very good pattern collections. The previous statement might not be true for all domains or for other restrictions than what we used in the statistical analysis, and as such, a more extensive statistical analysis should be carried out. Of course, we believe that we would get a similar results in most domains.

\section{CEGAR}

Variable projection is a fairly coarse abstraction method, and another method which allows for a more fine grained control is cartesian abstraction [SH13], and variable projection is a special case of it. Seipp and Helmert [SH13] use cartesian abstraction in roughly the following way: (1) find a solution for the abstract instance, if there is none then the original instance is unsolvable; (2) find a flaw in the solution by applying it on the original instance, if there is none then we have found a solution for the original instance; (3) adjust the abstraction to resolve the flaw; (4) if we are not satisfied with the current abstraction then go to (1), otherwise we are done. In other words, this algorithm, also known as counterexample-guided cartesian abstraction refinement (CEGAR), disproves abstract solutions. Every abstract solution for unsolvable instances can be disproven, however, CEGAR appears to not work well on unsolvable instances in practice (we got 74 out of 183 total coverage on the same machine and instances as our other experiments). 
This might be because there are simply too many abstract solutions to disprove, and disproving them one by one is too time consuming. In contrast to cartesian abstraction, the success of variable projection could be explained by being so coarse; it disproves a number of abstract solutions in one go. We envision that CEGAR could be useful towards the end of both consistency checking and the PDB methods: once we have identified a variable projection with a lot of dead-end states but which is solvable, there might be just a few abstract solutions to disprove and thus it might be appropriate to use CEGAR to find an unsolvable abstraction. In other words, if no unsolvable variable projection is found, then our best candidate could be used to jump-start CEGAR.

Additionally, PDBs could be used to help CEGAR decide how to resolve flaws by looking at the PDBs with many dead-ends. For example, suppose CEGAR constructs an abstract solution that visits a dead-end state. CEGAR would, eventually, refine the abstraction such that the dead-end state is detected, but if it had access to dead-end detection then it might be able to do so earlier by looking at the pattern. Alternatively, CEGAR could attempt to apply the abstract solution on the original instance during its construction. That is, for every action added to the abstract solution, apply the latest action and, by using some dead-end detection, check if the latest reached state is a dead-end. However, this must be done with care since CEGAR might backtrack, i.e. we do not want to preemptively fix flaws. The benefit is that CEGAR can avoid generating, and thus avoid disproving, abstract solutions that other techniques has already disproved. Note that any dead-end detection technique for the original instance can be used.

\section{Polytrees}

We showed that $\overline{\mathcal{P}}_{k, d}^{\infty, \infty}$ and $\overrightarrow{\mathcal{P}}_{k, d}^{i, \infty}$ are in $\mathbf{P}$. Suppose our goal is to find a more general and tractable class, then consider the following results for the PG problem.

- $\overline{\mathcal{P}}_{2, \infty}^{\infty, \infty}$ is NP-hard [GJ08], i.e. polytree causal graphs with binary variables;

- $\overrightarrow{\mathcal{P}}_{5, \infty}^{1,1}$ is NP-hard [GJ09], that is, we cannot remove the bound on the diameter or depth; and

- $\overline{\mathcal{P}}_{\infty, 2}^{\infty, \infty}$ is NP-hard [DD01], namely, we cannot remove the bound on both the indegree and the domain size. 
Two open questions are whether the problems $\overrightarrow{\mathcal{P}}_{k, d}^{\infty, \infty}$ and $\overrightarrow{\mathcal{P}}_{\infty, d}^{i, \infty}$ are tractable. Note that the first problem generalises both of our results, whilst the second generalises the last result. It might be difficult to adapt our algorithms to unbounded domain sizes since tractability follows from proving an upper bound on the number of times every variable has to change value. The derivation of the upper bound rely heavily on the maximum domain size, but is more flexible with respect to indegree. Hence, one way of showing that $\overrightarrow{\mathcal{P}}_{k, d}^{\infty, \infty}$ is tractable could be to remove the dependency on the indegree when derivating said upper bound.

The polynomial time algorithm for $\overrightarrow{\mathcal{P}}_{k, d}^{i, \infty}$ is a divide-and-conquer algorithm: it identifies a split variable in the causal graph, splits the instance in it, and solves the subproblems independently. However, for this to work, we have to decide on a sequence of actions $S$ with effects on the split variable. This strategy is not just applicable for polytree causal graphs, in fact, we can do this for any causal graphs with articulation points (removing one disconnects the weakly connected component it is in). Note that every action with an effect on an articulation point must be unary. The problem is that it is difficult to decide on $S$. One possible approach of deciding on $S$ is to use existing heuristic functions since they often solve some relaxed problem under the hood, and the solution might provide insight into which actions should be in $S$. If one decides on an $S$ successfully, then the reward is monumental since the size of the state transition graph has, at least, been cut in half; and in the best case (e.g. fork and inverted-fork causal graphs) the total size of each independent state transition graphs is $\mathcal{O}(|V| \cdot|A| \cdot k)$ where $k$ is the maximum domain size and $|V|$ is the number of variables. Clearly, assuming $|S|$ is not exponential in $|V|$, this is much better than $\mathcal{O}\left(k^{|V|}\right)$. However, certain fork and inverted-forked causal graphs requires $|S|$ to be exponential in $|V|$. Whilst the notion of instance splitting is very intruiging, the concept is poorly understood and must be studied further to assess the feasibility of it. 


\section{Bibliography}

[ABJS16] Meysam Aghighi, Christer Bäckström, Peter Jonsson, and Simon Ståhlberg. Analysing approximability and heuristics in planning using the exponential-time hypothesis. In Proceedings of the 22nd European Conference on Artificial Intelligence (ECAI'16), pages $184-192,2016$.

[ABJSb16] Meysam Aghighi, Christer Bäckström, Peter Jonsson, and Simon Ståhlberg. Refining complexity analyses in planning by exploiting the exponential time hypothesis. Annals of Mathematics and Artificial Intelligence (AMAI), pages 157$175,2016$.

[AJS15] Meysam Aghighi, Peter Jonsson, and Simon Ståhlberg. Tractable cost-optimal planning over restricted polytree causal graphs. In Proceedings of the 29th AAAI Conference on Artificial Intelligence (AAAI'15), pages 3225-3231, 2015 .

[App16] Austin Appleby. Murmurhash. https://github.com/ aappleby/smhasher/, January 2016.

[BD03] Ronen I. Brafman and Carmel Domshlak. Structure and complexity in planning with unary operators. Journal of Artificial Intelligence Research (JAIR), pages 315-349, 2003.

[BGHH05] Mark Boddy, Johnathan Gohde, Thomas Haigh, and Steven Harp. Course of action generation for cyber security using classical planning. In Proceedings of the 15th International Conference on Automated Planning and Scheduling (ICAPS '05), pages 12-21, 2005. 
[BJS13] Christer Bäckström, Peter Jonsson, and Simon Ståhlberg. Fast detection of unsolvable planning instances using local consistency. In Proceedings of the 6th International Symposium on Combinatorial Search (SoCS '13), pages 29-37, 2013.

[BN95] Christer Bäckström and Bernhard Nebel. Complexity results for SAS+ planning. Computational Intelligence, 11(4):625-655, 1995.

[CLRS09] Thomas H. Cormen, Charles E. Leiserson, Ronald L. Rivest, and Clifford Stein. Introduction to Algorithms, Third Edition. The MIT Press, 3rd edition, 2009.

[CS98] Joseph Culberson and Jonathan Schaeffer. Pattern databases. Computational Intelligence, 14(3):318-334, 1998.

[Dau07] Kerstin Dautenhahn. Socially intelligent robots: dimensions of human-robot interaction. Philosophical Transactions of the Royal Society B: Biological Sciences, 362(1480):679-704, 2007.

[DD01] Carmel Domshlak and Yefim Dinitz. Multi-agent off-line coordination: Structure and complexity. In 6th European Conference on Planning (ECP '01), pages 34-43, 2001.

[DF99] Rodney Downey and Michael Fellows. Parameterized Complexity. Monographs in Computer Science. Springer, 1999.

[ELV07] Stefan Edelkamp, Stefan Leue, and Willem Visser, editors. Directed Model Checking, 26.04. - 29.04.2006, volume 06172 of Dagstuhl Seminar Proceedings. Internationales Begegnungs- und Forschungszentrum fuer Informatik (IBFI), Schloss Dagstuhl, Germany, 2007.

[ERH17] Salomé Eriksson, Gabriele Röger, and Malte Helmert. Unsolvability certificates for classical planning. In Proceedings of the 27th International Conference on Automated Planning and Scheduling (ICAPS '17), pages 88-97, 2017.

$\left[\mathrm{FBCc}^{+} 10\right]$ David Ferrucci, Eric Brown, Jennifer Chu-carroll, James Fan, David Gondek, Aditya A. Kalyanpur, Adam Lally, J. William Murdock, Eric Nyberg, John Prager, Nico Schlaefer, and Chris Welty. Articles building Watson: An 
overview of the DeepQA project. AI magazine, 31(3):59-79, 2010 .

[Fer12] David Ferrucci. Introduction to "This is Watson". IBM Journal of Research and Development, 56(3):235-249, May 2012 .

[FG06] Jörg Flum and Martin Grohe. Parameterized Complexity Theory. Texts in Theoretical Computer Science. An EATCS Series. Springer, 2006.

[FKMH07] Ariel Felner, Richard E. Korf, Ram Meshulam, and Robert C. Holte. Compressed pattern databases. Journal of Artificial Intelligence (JAIR), 30:213-247, 2007.

[FLB $\left.{ }^{+} 13\right]$ David Ferrucci, Anthony Levas, Sugato Bagchi, David Gondek, and Erik Mueller. Watson: Beyond Jeopardy! Artificial Intelligence, 199-200(1):93-105, Jun 2013.

[FNRS10] Ariel Futoransky, Luciano Notarfrancesco, Gerardo Richarte, and Carlos Sarraute. Building computer network attacks. Computing Research Repository (CoRR), abs/1006.1916, 2010.

[GJ79] Michael Garey and David Johnson. Computers and Intractability: A Guide to the Theory of NP-Completeness. W. H. Freeman, 1979.

[GJ08] Omer Giménez and Anders Jonsson. The complexity of planning problems with simple causal graphs. Journal of Artificial Intelligence Research (JAIR), pages 319-351, 2008.

[GJ09] Omer Giménez and Anders Jonsson. Planning over chain causal graphs for variables with domains of size 5 is NPhard. Journal of Artificial Intelligence Research (JAIR), pages 675-706, 2009.

[GJ12] Omer Giménez and Anders Jonsson. The influence of kdependence on the complexity of planning. Artificial Intelligence, 177-179:25-45, 2012.

[GKS12] Robert Goldman, Ugur Kuter, and Tony Schneider. Using classical planners for plan verification and counterexample generation. AAAI Workshops, 2012. 
[GSH16] Daniel Gnad, Marcel Steinmetz, and Jörg Hoffmann. Django: Unchaining the power of red-black planning. First Unsolvability International Planning Competition, 2016.

[GSJ+16] Daniel Gnad, Marcel Steinmetz, Mathäus Jany, Jörg Hoffmann, Ivan Serina, and Alfonso Gerevini. Partial delete relaxation, unchained: On intractable red-black planning and its applications. In Proceedings of the 9th Annual Symposium on Combinatorial Search (SoCS '16), pages 45-53, 2016.

[GTSH17] Daniel Gnad, Álvaro Torralba, Alexander Shleyfman, and Jörg Hoffmann. Symmetry breaking in star-topology decoupled search. In Proceedings of the 27th International Conference on Automated Planning and Scheduling (ICAPS '17), pages 125-134, 2017.

[GW94] Ian Gent and Toby Walsh. The SAT phase transition. In Proceedings of the 11th European Conference on Artificial Intelligence (ECAI'94), pages 105-109, 1994.

$\left[\mathrm{HBH}^{+} 07\right]$ Patrik Haslum, Adi Botea, Malte Helmert, Blai Bonet, and Sven Koenig. Domain-independent construction of pattern database heuristics for cost-optimal planning. In Proceedings of the 22th AAAI Conference on Artificial Intelligence (AAAI '07), pages 1007-1012, 2007.

[HD09] Malte Helmert and Carmel Domshlak. Landmarks, critical paths and abstractions: What's the difference anyway? In Proceedings of the 19th International Conference on Automated Planning and Scheduling (ICAPS '09), pages 162-169, 2009.

[Hel04] Malte Helmert. A planning heuristic based on causal graph analysis. In Proceedings of the 14th International Conference on Automated Planning and Scheduling (ICAPS '04), pages 161-170, 2004.

[Hel06a] Malte Helmert. The Fast Downward planning system. Journal of Artificial Intelligence Research (JAIR), 26:191$246,2006$. 
[Hel06b] Malte Helmert. New complexity results for classical planning benchmarks. In Proceedings of the 16th International Conference on Automated Planning and Scheduling (ICAPS '06), pages 52-61, 2006.

[HG00] Patrik Haslum and Héctor Geffner. Admissible heuristics for optimal planning. In Proceedings of 5th International Conference on Artificial Intelligence Planning and Scheduling (AIPS '00, pages 140-149, 2000.

[HGHB05] Steven Harp, Johnathan Gohde, Thomas Haigh, and Mark Boddy. Automated vulnerability analysis using AI planning. In AI Technologies for Homeland Security, Papers from the 2005 AAAI Spring Symposium, Technical Report SS-05-01, Stanford, California, USA, March 21-23, 2005, pages 46-53, 2005.

[HHH07] Malte Helmert, Patrik Haslum, and Jörg Hoffmann. Flexible abstraction heuristics for optimal sequential planning. In Proceedings of the 17th International Conference on Automated Planning and Scheduling (ICAPS '07), pages 176-183, 2007.

[HJ00] Patrik Haslum and Peter Jonsson. Planning with reduced operator sets. In Proceedings of 5th International Conference on Artificial Intelligence Planning and Scheduling (AIPS '00), pages 150-158, 2000.

[HKT14] Jörg Hoffmann, Peter Kissmann, and Álvaro Torralba. "Distance"? Who cares? Tailoring merge-and-shrink heuristics to detect unsolvability. In Proceedings of the 21st European Conference on Artificial Intelligence (ECAI '14), pages 441446, 2014.

[HN01] Jörg Hoffmann and Bernhard Nebel. The FF planning system: Fast plan generation through heuristic search. Journal of Artificial Intelligence Research (JAIR), 14:253-302, 2001.

[HNR68] Peter Hart, Nils Nilsson, and Bertram Raphael. A formal basis for the heuristic determination of minimum cost paths. IEEE Transactions on Systems, Science, and Cybernetics, 4(2):100-107, 1968. 
[IP01] Russell Impagliazzo and Ramamohan Paturi. On the complexity of k-SAT. Journal of Computer and System Sciences (JCSS), 62(2):367-375, 2001.

[IPZ01] Russell Impagliazzo, Ramamohan Paturi, and Francis Zane. Which problems have strongly exponential complexity? Journal of Computer and System Sciences (JCSS), 63(4):512-530, 2001.

$\left[\mathrm{JMM}^{+} 00\right]$ Ari Jónsson, Paul Morris, Nicola Muscettola, Kanna Rajan, and Ben Smith. Planning in interplanetary space: Theory and practice. In Proceedings of the 5th International Conference on Artificial Intelligence Planning Systems (AIPS '00), pages $177-186,2000$.

[JW02] Glen Jeh and Jennifer Widom. SimRank: A measure of structural-context similarity. In Proceedings of the 8th ACM SIGKDD International Conference on Knowledge Discovery and Data Mining (KDD '02), pages 538-543, 2002.

$\left[\mathrm{KAK}^{+} 97\right]$ Hiroaki Kitano, Minoru Asada, Yasuo Kuniyoshi, Itsuki Noda, and Eiichi Osawa. RoboCup: The robot world cup initiative. In Proceedings of the First International Conference on Autonomous Agents, pages 340-347, 1997.

[KD07] Michael Katz and Carmel Domshlak. Structural patterns of tractable sequentially-optimal planning. In 17th International Conference on Automated Planning and Scheduling (ICAPS '07), pages 200-207, 2007.

[KD08a] Michael Katz and Carmel Domshlak. New islands of tractability of cost-optimal planning. Journal of Artificial Intelligence Research (JAIR), 32(1):203-288, 2008.

[KD08b] Michael Katz and Carmel Domshlak. Structural patterns heuristics via fork decomposition. In 18th International Conference on Automated Planning and Scheduling (ICAPS '08), pages 182-189, 2008.

[KD09] Michael Katz and Carmel Domshlak. Structural-pattern databases. In 19th International Conference on Automated Planning and Scheduling (ICAPS '09), pages 186-193, 2009. 
[KD10] Michael Katz and Carmel Domshlak. Implicit abstraction heuristics. Journal of Artificial Intelligence Research (JAIR), 39:51-126, 2010.

[KK12] Michael Katz and Emil Keyder. Structural patterns beyond forks: Extending the complexity boundaries of classical planning. In 26th AAAI Conference on Artificial Intelligence (AAAI '12), pages 1779-1785, 2012.

[Kno94] Craig Knoblock. Automatically generating abstractions for planning. Artificial Intelligence, 68:243-302, 1994.

[LCO15] Carlos Linares López, Sergio Jiménez Celorrio, and Ángel García Olaya. The deterministic part of the seventh international planning competition. Artificial Intelligence, 223:82-119, 2015.

[LMG16] Nir Lipovetzky, Christian Muise, and Hector Geffner. Traps, invariants, and dead-ends. In Proceedings of the 26th International Conference on Automated Planning and Scheduling (ICAPS '16), pages 211-215, 2016.

[Moo59] Edward Moore. The shortest path through a maze. In Proceedings of the International Symposium on the Theory of Switching, pages 285-292. Harvard University Press, 1959.

[Orm16] David Ormerod. AlphaGo defeats Lee Sedol 4-1 in Google DeepMind challenge match. https://gogameguru.com/ alphago-defeats-lee-sedol-4-1/, March 2016.

[Pan97] Bruce Pandolfini. Kasparov and Deep Blue: The Historic Chess Match Between Man and Machine. Fireside Chess Library, 1997.

[Pap94] Christos H. Papadimitriou. Computational Complexity. Addison-Wesley, 1994.

[PRB14] André Grahl Pereira, Marcus Ritt, and Luciana S. Buriol. Solving sokoban optimally using pattern databases for deadlock detection. In Encontro Nacional de Inteligência Artificial e Computacional, pages 514-520, 2014.

[PS16] Florian Pommerening and Jendrik Seipp. Fast downward dead-end pattern database. First Unsolvability International Planning Competition, 2016. 
[RHN06] Jussi Rintanen, Keijo Heljanko, and Ilkka Niemelä. Planning as satisfiability: parallel plans and algorithms for plan search. Artificial Intelligence, 170(12-13):1031-1080, 2006.

[RMKB13] Hayley Robinson, Bruce MacDonald, Ngaire Kerse, and Elizabeth Broadbent. The psychosocial effects of a companion robot: A randomized controlled trial. Journal of the American Medical Directors Association, 14(9):661 - 667, 2013.

[RW10] Silvia Richter and Matthias Westphal. The LAMA planner: Guiding cost-based anytime planning with landmarks. Journal of Artificial Intelligence Research (JAIR), 39(1):127177, September 2010.

[SBH13] Carlos Sarraute, Olivier Buffet, and Jörg Hoffmann. Pomdps make better hackers: Accounting for uncertainty in penetration testing. Computing Research Repository (CoRR), abs/1307.8182, 2013.

[SH13] Jendrik Seipp and Malte Helmert. Counterexampleguided cartesian abstraction refinement. In 23rd International Conference on Automated Planning and Scheduling (ICAPS '13), pages 347-351, 2013.

[SH16] Marcel Steinmetz and Jörg Hoffmann. Towards clauselearning state space search: Learning to recognize dead-ends. In Proceedings of the 30th AAAI Conference on Artificial Intelligence (AAAI'16), pages 760-768, 2016.

$\left[\mathrm{SHM}^{+} 16\right]$ David Silver, Aja Huang, Chris Maddison, Arthur Guez, Laurent Sifre, George van den Driessche, Julian Schrittwieser, Ioannis Antonoglou, Veda Panneershelvam, Marc Lanctot, Sander Dieleman, Dominik Grewe, John Nham, Nal Kalchbrenner, Ilya Sutskever, Timothy Lillicrap, Madeleine Leach, Koray Kavukcuoglu, Thore Graepel, and Demis Hassabis. Mastering the game of go with deep neural networks and tree search. Nature, 529(7587):454-489, January 2016.

[Smi04] David Smith. Choosing objectives in over-subscription planning. In Proceedings of the 14th International Conference 
on Automated Planning and Scheduling (ICAPS '04), pages 393-401, 2004.

[SP12] Carlos Sarraute and Ken Pickering. Encounters of the third kind between pentesting and automated planning. Invited talk at AAAI '12 workshop Problem Solving Using Classical Planners, 2012.

[SPS ${ }^{+}$16] Jendrik Seipp, Florian Pommerening, Silvan Sievers, Martin Wehrle, Chris Fawcett, and Yusra Alkhazraji. Fast Downward Aidos. First Unsolvability International Planning Competition, 2016.

[Stå17] Simon Ståhlberg. Tailoring pattern databases for unsolvable planning instances. In Proceedings of the 27th International Conference on Automated Planning and Scheduling (ICAPS '17), pages 274-282, 2017.

[THK16] Álvaro Torralba, Jörg Hoffmann, and Peter Kissmann. MSUnsat and SimulationDominance: Merge-and-shrink and dominance pruning for proving unsolvability. First Unsolvability International Planning Competition, 2016.

[Tor16] Álvaro Torralba. SymPA: Symbolic perimeter abstractions for proving unsolvability. First Unsolvability International Planning Competition, 2016.

[Tré82] Charles Pierre Trémaux. French engineer of the telegraph in public conference. In École polytechnique of Paris, 18591882 .

[Tur50] Alan Turing. Computing machinery and intelligence. Mind, 59(236):433-460, 1950.

[Vyg11] Jens Vygen. Splitting trees at vertices. Discrete Mathematics, 311(1):67-69, 2011.

$\left[\mathrm{ZTW}^{+}\right.$09] Zhiping Zeng, Anthony K. H. Tung, Jianyong Wang, Jianhua Feng, and Lizhu Zhou. Comparing stars: On approximating graph edit distance. In Proceedings of the $V L D B$ Endowment, 2(1):25-36, August 2009. 



\section{Dissertations}

\section{Linköping Studies in Science and Technology Linköping Studies in Arts and Science Linköping Studies in Statistics \\ Linköping Studies in Information Science}

\section{Linköping Studies in Science and Technology}

No 14 Anders Haraldsson: A Program Manipulation System Based on Partial Evaluation, 1977, ISBN 917372-144-1.

No 17 Bengt Magnhagen: Probability Based Verification of Time Margins in Digital Designs, 1977, ISBN 91-7372157-3.

No 18 Mats Cedwall: Semantisk analys av processbeskrivningar i naturligt språk, 1977, ISBN 91- 7372168-9.

No 22 Jaak Urmi: A Machine Independent LISP Compiler and its Implications for Ideal Hardware, 1978, ISBN 91-7372-188-3.

No 33 Tore Risch: Compilation of Multiple File Queries in a Meta-Database System 1978, ISBN 91- 7372-232-4.

No 51 Erland Jungert: Synthesizing Database Structures from a User Oriented Data Model, 1980, ISBN 917372-387-8.

No 54 Sture Hägglund: Contributions to the Development of Methods and Tools for Interactive Design of Applications Software, 1980, ISBN 91-7372-404-1.

No 55 Pär Emanuelson: Performance Enhancement in a Well-Structured Pattern Matcher through Partial Evaluation, 1980, ISBN 91-7372-403-3.

No 58 Bengt Johnsson, Bertil Andersson: The HumanComputer Interface in Commercial Systems, 1981, ISBN 91-7372-414-9.

No 69 H. Jan Komorowski: A Specification of an Abstract Prolog Machine and its Application to Partial Evaluation, 1981, ISBN 91-7372-479-3.

No 71 René Reboh: Knowledge Engineering Techniques and Tools for Expert Systems, 1981, ISBN 91-7372489-0.

No 77 Östen Oskarsson: Mechanisms of Modifiability in large Softw are Systems, 1982, ISBN 91- 7372-527-7.

No 94 Hans Lunell: Code Generator Writing Systems, 1983, ISBN 91-7372-652-4.

No 97 Andrzej Lingas: Advances in Minimum Weight Triangulation, 1983, ISBN 91-7372-660-5.

No 109 Peter Fritzson: Tow ards a Distributed Programming Environment based on Incremental Compilation, 1984, ISBN 91-7372-801-2.

No 111 Erik Tengvald: The Design of Expert Planning Systems. An Experimental Operations Planning System for Turning, 1984, ISBN 91-7372- 805-5.

No 155 Christos Levcopoulos: Heuristics for Minimum Decompositions of Polygons, 1987, ISBN 91-7870133-3.

No 165 James W. Goodwin: A Theory and System for NonMonotonic Reasoning, 1987, ISBN 91-7870-183-X.

No 170 Zebo Peng: A Formal Methodology for Automated Synthesis of VLSI Systems, 1987, ISBN 91-7870-225-9.

No 174 Johan Fagerström: A Paradigm and System for Design of Distributed Systems, 1988, ISBN 91-7870301-8.

No 192 Dimiter Driankov: Tow ards a Many Valued Logic of Quantified Belief, 1988, ISBN 91-7870-374-3.
No 213 Lin Padgham: Non-Monotonic Inheritance for an Object Oriented Knowledge Base, 1989, ISBN 917870-485-5.

No 214 Tony Larsson: A Formal Hardware Description and Verification Method, 1989, ISBN 91-7870-517-7.

No 221 Michael Reinfrank: Fundamentals and Logical Foundations of Truth Maintenance, 1989, ISBN 917870-546-0.

No 239 Jonas Löwgren: Knowledge-Based Design Support and Discourse Management in User Interface Management Systems, 1991, ISBN 91-7870-720-X.

No 244 Henrik Eriksson: Meta-Tool Support for Knowledge Acquisition, 1991, ISBN 91-7870-746-3.

No 252 Peter Eklund: An Epistemic Approach to Interactive Design in Multiple Inheritance Hierarchies, 1991, ISBN 91-7870-784-6.

No 258 Patrick Doherty: NML3 - A Non-Monotonic Formalism with Explicit Defaults, 1991, ISBN 917870-816-8.

No 260 Nahid Shahmehri: Generalized Algorithmic Debugging, 1991, ISBN 91-7870-828-1.

No 264 Nils Dahlbäck: Representation of DiscourseCognitive and Computational Aspects, 1992, ISBN 91-7870-850-8.

No 265 Ulf Nilsson: Abstract Interpretations and Abstract Machines: Contributions to a Methodology for the Implementation of Logic Programs, 1992, ISBN 917870-858-3.

No 270 Ralph Rönnquist: Theory and Practice of Tensebound Object References, 1992, ISBN 91-7870-873-7.

No 273 Björn Fjellborg: Pipeline Extraction for VLSI Data Path Synthesis, 1992, ISBN 91-7870-880-X.

No 276 Staffan Bonnier: A Formal Basis for Horn Clause Logic with External Polymorphic Functions, 1992, ISBN 91-7870-896-6.

No 277 Kristian Sandahl: Developing Knowledge Management Systems with an Active Expert Methodology, 1992, ISBN 91-7870-897-4.

No 281 Christer Bäckström: Computational Complexity of Reasoning about Plans, 1992, ISBN 91-7870-979-2.

No 292 Mats Wirén: Studies in Incremental Natural Language Analysis, 1992, ISBN 91-7871-027-8.

No 297 Mariam Kamkar: Interprocedural Dynamic Slicing with Applications to Debugging and Testing, 1993, ISBN 91-7871-065-0.

No 302 Tingting Zhang: A Study in Diagnosis Using Classification and Defaults, 1993, ISBN 91-7871-078-2

No 312 Arne Jönsson: Dialogue Management for Natural Language Interfaces - An Empirical Approach, 1993, ISBN 91-7871-110-X.

No 338 Simin Nadjm-Tehrani: Reactive Systems in Physical Environments: Compositional Modelling and Framework for Verification, 1994, ISBN 91-7871-237-8.

No 371 Bengt Savén: Business Models for Decision Support and Learning. A Study of Discrete-Event Manufacturing Simulation at Asea/ ABB 1968-1993, 1995, ISBN 91-7871-494-X. 
No 375 Ulf Söderman: Conceptual Modelling of Mode Switching Physical Systems, 1995, ISBN 91-7871-5164.

No 383 Andreas Kågedal: Exploiting Groundness in Logic Programs, 1995, ISBN 91-7871-538-5.

No 396 George Fodor: Ontological Control, Description, Identification and Recovery from Problematic Control Situations, 1995, ISBN 91-7871-603-9.

No 413 Mikael Pettersson: Compiling Natural Semantics, 1995, ISBN 91-7871-641-1.

No 414 Xinli Gu: RT Level Testability Improvement by Testability Analysis and Transformations, 1996, ISBN 91-7871-654-3.

No 416 Hua Shu: Distributed Default Reasoning, 1996, ISBN 91-7871-665-9.

No 429 Jaime Villegas: Simulation Supported Industrial Training from an Organisational Learning Perspective - Development and Evaluation of the SSIT Method, 1996, ISBN 91-7871-700-0.

No 431 Peter Jonsson: Studies in Action Planning: Algorithms and Complexity, 1996, ISBN 91-7871-7043.

No 437 Johan Boye: Directional Types in Logic Programming, 1996, ISBN 91-7871-725-6.

No 439 Cecilia Sjöberg: Activities, Voices and Arenas: Participatory Design in Practice, 1996, ISBN 91-7871728-0.

No 448 Patrick Lambrix: Part-Whole Reasoning in Description Logics, 1996, ISBN 91-7871-820-1.

No 452 Kjell Orsborn: On Extensible and Object-Relational Database Technology for Finite Element Analysis Applications, 1996, ISBN 91-7871-827-9.

No 459 Olof Johansson: Development Environments for Complex Product Models, 1996, ISBN 91-7871-855-4.

No 461 Lena Strömbäck: User-Defined Constructions in Unification-Based Formalisms, 1997, ISBN 91-7871857-0.

No 462 Lars Degerstedt: Tabulation-based Logic Programming: A Multi-Level View of Query Answering, 1996, ISBN 91-7871-858-9.

No 475 Fredrik Nilsson: Strategi och ekonomisk styrning En studie av hur ekonomiska styrsystem utformas och används efter företagsförvärv, 1997, ISBN 917871-914-3.

No 480 Mikael Lindvall: An Empirical Study of Requirements-Driven Impact Analysis in Object-Oriented Softw are Evolution, 1997, ISBN 91-7871-927-5.

No 485 Göran Forslund: Opinion-Based Systems: The Cooperative Perspective on Knowledge-Based Decision Support, 1997, ISBN 91-7871-938-0.

No 494 Martin Sköld: Active Database Management Systems for Monitoring and Control, 1997, ISBN 917219-002-7.

No 495 Hans Olsén: Automatic Verification of Petri Nets in a CLP framew ork, 1997, ISBN 91-7219-011-6.

No 498 Thomas Drakengren: Algorithms and Complexity for Temporal and Spatial Formalisms, 1997, ISBN 917219-019-1.

No 502 Jakob Axelsson: Analysis and Synthesis of Heterogeneou s Real-Time Systems, 1997, ISBN 91-7219-035-3.

No 503 Johan Ringström: Compiler Generation for DataParallel Programming Languages from Two-Level Semantics Specifications, 1997, ISBN 91-7219-045-0.

No 512 Anna Moberg: Närhet och distans - Studier av kommunikationsmönster i satellitkontor och flexibla kontor, 1997, ISBN 91-7219-119-8.
No 520 Mikael Ronström: Design and Modelling of a Parallel Data Server for Telecom Applications, 1998, ISBN 91-7219-169-4.

No 522 Niclas Ohlsson: Tow ards Effective Fault Prevention - An Empirical Study in Software Engineering, 1998, ISBN 91-7219-176-7.

No 526 Joachim Karlsson: A Systematic Approach for Prioritizing Software Requirements, 1998, ISBN 917219-184-8.

No 530 Henrik Nilsson: Declarative Debugging for Lazy Functional Languages, 1998, ISBN 91-7219-197-x.

No 555 Jonas Hallberg: Timing Issues in High-Level Synthesis, 1998, ISBN 91-7219-369-7.

No 561 Ling Lin: Management of 1-D Sequence Data - From Discrete to Continu ous, 1999, ISBN 91-7219-402-2.

No 563 Eva L Ragnemalm: Student Modelling based on Collaborative Dialogue with a Learning Companion, 1999, ISBN 91-7219-412-X.

No 567 Jörgen Lindström: Does Distance matter? On geographical dispersion in organisations, 1999, ISBN 917219-439-1.

No 582 Vanja Josifovski: Design, Implementation and Evaluation of a Distributed Mediator System for Data Integration, 1999, ISBN 91-7219-482-0.

No 589 Rita Kovordányi: Modeling and Simulating Inhibitory Mechanisms in Mental Image Reinterpretation - Towards Cooperative HumanComputer Creativity, 1999, ISBN 91-7219-506-1.

No 592 Mikael Ericsson: Supporting the Use of Design Knowledge - An Assessment of Commenting Agents, 1999, ISBN 91-7219-532-0.

No 593 Lars Karlsson: Actions, Interactions and Narratives, 1999, ISBN 91-7219-534-7.

No 594 C. G. Mikael Johansson: Social and Organizational Aspects of Requirements Engineering Methods - A practice-oriented approach, 1999, ISBN 91-7219-541$\mathrm{X}$.

No 595 Jörgen Hansson: Value-Driven Multi-Class Overload Management in Real-Time Database Systems, 1999, ISBN 91-7219-542-8.

No 596 Niklas Hallberg: Incorporating User Values in the Design of Information Systems and Services in the Public Sector: A Methods Approach, 1999, ISBN 91 7219-543-6.

No 597 Vivian Vimarlund: An Economic Perspective on the Analysis of Impacts of Information Technology: From Case Studies in Health-Care tow ards General Models and Theories, 1999, ISBN 91-7219-544-4.

No 598 Johan Jenvald: Methods and Tools in ComputerSupported Taskforce Training, 1999, ISBN 91-7219547-9.

No 607 Magnus Merkel: Understanding and enhancing translation by parallel text processing, 1999, ISBN 917219-614-9.

No 611 Silvia Coradeschi: Anchoring symbols to sensory data, 1999, ISBN 91-7219-623-8.

No 613 Man Lin: Analysis and Synthesis of Reactive Systems: A Generic Layered Architecture Perspective, 1999, ISBN 91-7219-630-0.

No 618 Jimmy Tjäder: Systemimplementering i praktiken En studie av logiker i fyra projekt, 1999, ISBN 917219-657-2.

No 627 Vadim Engelson: Tools for Design, Interactive Simulation, and Visualization of Object-Oriented Models in Scientific Computing, 2000, ISBN 91-7219709-9. 
No 637 Esa Falkenroth: Database Technology for Control and Simulation, 2000, ISBN 91-7219-766-8.

No 639 Per-Arne Persson: Bringing Power and Knowledge Together: Information Systems Design for Autonomy and Control in Command Work, 2000, ISBN 91-7219796-X.

No 660 Erik Larsson: An Integrated System-Level Design for Testability Methodology, 2000, ISBN 91-7219-890-7.

No 688 Marcus Bjäreland: Model-based Execution Monitoring, 2001, ISBN 91-7373-016-5.

No 689 Joakim Gustafsson: Extending Temporal Action Logic, 2001, ISBN 91-7373-017-3.

No 720 Carl-Johan Petri: Organizational Information Provision - Managing Mandatory and Discretionary Use of Information Technology, 2001, ISBN-91-7373-1269.

No 724 Paul Scerri: Designing Agents for Systems with Adjustable Autonomy, 2001, ISBN 9173732079.

No 725 Tim Heyer: Semantic Inspection of Software Artifacts: From Theory to Practice, 2001, ISBN 91 73732087.

No 726 Pär Carlshamre: A Usability Perspective on Requirements Engineering - From Methodology to Product Development, 2001, ISBN 9173732125.

No 732 Juha Takkinen: From Information Management to Task Management in Electronic Mail, 2002, ISBN 91 73732583.

No 745 Johan Åberg: Live Help Systems: An Approach to Intelligent Help for Web Information Systems, 2002, ISBN 91-7373-311-3.

No 746 Rego Granlund: Monitoring Distributed Teamwork Training, 2002, ISBN 91-7373-312-1.

No 757 Henrik André-Jönsson: Indexing Strategies for Time Series Data, 2002, ISBN 917373-346-6.

No 747 Anneli Hagdahl: Development of IT-supported Interorganisational Collaboration - A Case Study in the Swedish Public Sector, 2002, ISBN 91-7373-314-8.

No 749 Sofie Pilemalm: Information Technology for NonProfit Organisations - Extended Participatory Design of an Information System for Trade Union Shop Stew ard s, 2002, ISBN 91-7373-318-0.

No 765 Stefan Holmlid: Adapting users: Towards a theory of use quality, 2002, ISBN 91-7373-397-0.

No 771 Magnus Morin: Multimedia Representations of Distributed Tactical Operations, 2002, ISBN 91-7373-4217.

No 772 Pawel Pietrzak: A Type-Based Framew ork for Locating Errors in Constraint Logic Programs, 2002, ISBN 91-7373-422-5.

No 758 Erik Berglund: Library Communication Among Programmers Worldwide, 2002, ISBN 91-7373-349-0.

No 774 Choong-ho Yi: Modelling Object-Oriented Dynamic Systems Using a Logic-Based Framew ork, 2002, ISBN 91-7373-424-1.

No 779 Mathias Broxvall: A Study in the Computational Complexity of Temporal Reasoning, 2002, ISBN 917373-440-3.

No 793 Asmus Pandikow: A Generic Principle for Enabling Interoperability of Structured and Object-Oriented Analysis and Design Tools, 2002, ISBN 91-7373-479-9.

No 785 Lars Hult: Publika Informationstjänster. En studie av den Internetbaserade encyklopedins bruksegenskaper, 2003, ISBN 91-7373-461-6.

No 800 Lars Taxén: A Framework for the Coordination of Complex Systems' Development, 2003, ISBN 917373-604-X
No 808 Klas Gäre: Tre perspektiv på förväntningar och förändringar i samband med införande av informationssystem, 2003, ISBN 91-7373-618-X.

No 821 Mikael Kindborg: Concurrent Comics programming of social agents by children, 2003, ISBN 91-7373-651-1.

No 823 Christina Ölvingson: On Development of Information Systems with GIS Functionality in Public Health Informatics: A Requirements Engineering Approach, 2003, ISBN 91-7373-656-2.

No 828 Tobias Ritzau: Memory Efficient Hard Real-Time Garbage Collection, 2003, ISBN 91-7373-666-X.

No 833 Paul Pop: Analysis and Synthesis of Communication-Intensive Heterogeneous Real-Time Systems, 2003, ISBN 91-7373-683-X.

No 852 Johan Moe: Observing the Dynamic Behaviour of Large Distributed Systems to Improve Development and Testing - An Empirical Study in Software Engineering, 2003, ISBN 91-7373-779-8.

No 867 Erik Herzog: An Approach to Systems Engineering Tool Data Representation and Exchange, 2004, ISBN 91-7373-929-4.

No 872 Aseel Berglund: Augmenting the Remote Control: Studies in Complex Information Navigation for Digital TV, 2004, ISBN 91-7373-940-5.

No 869 Jo Skåmedal: Telecommuting's Implications on Travel and Travel Patterns, 2004, ISBN 91-7373-935-9.

No 870 Linda Askenäs: The Roles of IT - Studies of Organising when Implementing and Using Enterprise Systems, 2004, ISBN 91-7373-936-7.

No 874 Annika Flycht-Eriksson: Design and Use of Ontologies in Information-Providing Dialogue Systems, 2004, ISBN 91-7373-947-2.

No 873 Peter Bunus: Debugging Techniques for EquationBased Languages, 2004, ISBN 91-7373-941-3.

No 876 Jonas Mellin: Resource-Predictable and Efficient Monitoring of Events, 2004, ISBN 91-7373-956-1.

No 883 Magnus Bång: Computing at the Speed of Paper: Ubiquitous Computing Environments for Healthcare Professionals, 2004, ISBN 91-7373-971-5

No 882 Robert Eklund: Disfluency in Swedish humanhuman and human-machine travel booking dialogues, 2004, ISBN 91-7373-966-9.

No 887 Anders Lindström: English and other Foreign Linguistic Elements in Spoken Swedish. Studies of Productive Processes and their Modelling using Finite-State Tools, 2004, ISBN 91-7373-981-2.

No 889 Zhiping Wang: Capacity-Constrained Production-inventory systems - Modelling and Analysis in both a traditional and an e-business context, 2004, ISBN 9185295-08-6.

No 893 Pernilla Qvarfordt: Eyes on Multimodal Interaction, 2004, ISBN 91-85295-30-2.

No 910 Magnus Kald: In the Borderland between Strategy and Management Control - Theoretical Framework and Empirical Evidence, 2004, ISBN 91-85295-82-5.

No 918 Jonas Lundberg: Shaping Electronic News: Genre Perspectives on Interaction Design, 2004, ISBN 9185297-14-3.

No 900 Mattias Arvola: Shades of use: The dynamics of interaction design for sociable use, 2004, ISBN 9185295-42-6.

No 920 Luis Alejandro Cortés: Verification and Scheduling Techniques for Real-Time Embedded Systems, 2004, ISBN 91-85297-21-6.

No 929 Diana Szentivanyi: Performance Studies of FaultTolerant Middlew are, 2005, ISBN 91-85297-58-5. 
No 933 Mikael Cäker: Management Accounting as Constructing and Opposing Customer Focus: Three Case Studies on Management Accounting and Customer Relations, 2005, ISBN 91-85297-64-X.

No 937 Jonas Kvarnström: TALplanner and Other Extensions to Temporal Action Logic, 2005, ISBN 9185297-75-5.

No 938 Bourhane Kadmiry: Fuzzy Gain-Scheduled Visual Servoing for Unmanned Helicopter, 2005, ISBN 9185297-76-3.

No 945 Gert Jervan: Hybrid Built-In Self-Test and Test Generation Techniques for Digital Systems, 2005, ISBN : 91-85297-97-6.

No 946 Anders Arpteg: Intelligent Semi-Structured Information Extraction, 2005, ISBN 91-85297-98-4.

No 947 Ola Angelsmark: Constructing Algorithms for Constraint Satisfaction and Related Problems - Methods and Applications, 2005, ISBN 91-85297-99-2.

No 963 Calin Curescu: Utility-based Optimisation of Resource Allocation for Wireless Networks, 2005, ISBN 91-85457-07-8.

No 972 Björn Johansson: Joint Control in Dynamic Situations, 2005, ISBN 91-85457-31-0.

No 974 Dan Lawesson: An Approach to Diagnosability Analysis for Interacting Finite State Systems, 2005, ISBN 91-85457-39-6.

No 979 Claudiu Duma: Security and Trust Mechanisms for Groups in Distributed Services, 2005, ISBN 91-8545754-X.

No 983 Sorin Manolache: Analysis and Optimisation of Real-Time Systems with Stochastic Behaviour, 2005, ISBN 91-85457-60-4.

No 986 Yuxiao Zhao: Standards-Based Application Integration for Business-to-Business Communications, 2005, ISBN 91-85457-66-3.

No 1004 Patrik Haslum: Admissible Heuristics for Automated Planning, 2006, ISBN 91-85497-28-2.

No 1005 Aleksandra Tešanovic: Developing Reusable and Reconfigurable Real-Time Software using Aspects and Components, 2006, ISBN 91-85497-29-0.

No 1008 David Dinka: Role, Identity and Work: Extending the design and development agenda, 2006, ISBN 91$85497-42-8$.

No 1009 Iakov Nakhimovski: Contributions to the Modeling and Simulation of Mechanical Systems with Detailed Contact Analysis, 2006, ISBN 91-85497-43-X.

No 1013 Wilhelm Dahllöf: Exact Algorithms for Exact Satisfiability Problems, 2006, ISBN 91-85523-97-6.

No 1016 Levon Saldamli: PDEModelica - A High-Level Language for Modeling with Partial Differential Equations, 2006, ISBN 91-85523-84-4.

No 1017 Daniel Karlsson: Verification of Component-based Embedded System Designs, 2006, ISBN 91-85523-79-8

No 1018 Ioan Chisalita: Communication and Networking Techniques for Traffic Safety Systems, 2006, ISBN 9185523-77-1.

No 1019 Tarja Susi: The Puzzle of Social Activity - The Significance of Tools in Cognition and Cooperation, 2006, ISBN 91-85523-71-2

No 1021 Andrzej Bednarski: Integrated Optimal Code Generation for Digital Signal Processors, 2006, ISBN 9185523-69-0.

No 1022 Peter Aronsson: Automatic Parallelization of Equation-Based Simulation Programs, 2006, ISBN 9185523-68-2.
No 1030 Robert Nilsson: A Mutation-based Framework for Automated Testing of Timeliness, 2006, ISBN 9185523-35-6.

No 1034 Jon Edvardsson: Techniques for Automatic Generation of Tests from Programs and Specifications, 2006, ISBN 91-85523-31-3.

No 1035 Vaida Jakoniene: Integration of Biological Data, 2006, ISBN 91-85523-28-3.

No 1045 Genevieve Gorrell: Generalized Hebbian Algorithms for Dimensionality Reduction in Natural Language Processing, 2006, ISBN 91-85643-88-2.

No 1051 Yu-Hsing Huang: Having a New Pair of Glasses Applying Systemic Accident Models on Road Safety, 2006, ISBN 91-85643-64-5.

No 1054 Åsa Hedenskog: Perceive those things which cannot be seen - A Cognitive Systems Engineering perspective on requirements management, 2006, ISBN 91-85643-57-2.

No 1061 Cécile Åberg: An Evaluation Platform for Semantic Web Technology, 2007, ISBN 91-85643-31-9.

No 1073 Mats Grindal: Handling Combinatorial Explosion in Softw are Testing, 2007, ISBN 978-91-85715-74-9.

No 1075 Almut Herzog: Usable Security Policies for Runtime Environments, 2007, ISBN 978-91-85715-65-7.

No 1079 Magnus Wahlström: Algorithms, measures, and upper bounds for Satisfiability and related problems, 2007, ISBN 978-91-85715-55-8.

No 1083 Jesper Andersson: Dynamic Software Architectures, 2007, ISBN 978-91-85715-46-6.

No 1086 Ulf Johansson: Obtaining Accurate and Comprehensible Data Mining Models - An Evolutionary Approach, 2007, ISBN 978-91-85715-34-3.

No 1089 Traian Pop: Analysis and Optimisation of Distributed Embedded Systems with Heterogeneous Scheduling Policies, 2007, ISBN 978-91-85715-27-5.

No 1091 Gustav Nordh: Complexity Dichotomies for CSPrelated Problems, 2007, ISBN 978-91-85715-20-6.

No 1106 Per Ola Kristensson: Discrete and Continuous Shape Writing for Text Entry and Control, 2007, ISBN 97891-85831-77-7.

No 1110 He Tan: Aligning Biomedical Ontologies, 2007, ISBN 978-91-85831-56-2.

No 1112 Jessica Lindblom: Minding the body - Interacting socially through embodied action, 2007, ISBN 978-9185831-48-7.

No 1113 Pontus Wärnestål: Dialogue Behavior Management in Conversational Recommender Systems, 2007, ISBN 978-91-85831-47-0.

No 1120 Thomas Gustafsson: Management of Real-Time Data Consistency and Transient Overloads in Embedded Systems, 2007, ISBN 978-91-85831-33-3.

No 1127 Alexandru Andrei: Energy Efficient and Predictable Design of Real-time Embedded Systems, 2007, ISBN 978-91-85831-06-7.

No 1139 Per Wikberg: Eliciting Knowledge from Experts in Modeling of Complex Systems: Managing Variation and Interactions, 2007, ISBN 978-91-85895-66-3.

No 1143 Mehdi Amirijoo: QoS Control of Real-Time Data Services under Uncertain Workload, 2007, ISBN 97891-85895-49-6.

No 1150 Sanny Syberfeldt: Optimistic Replication with Forward Conflict Resolution in Distributed Real-Time Databases, 2007, ISBN 978-91-85895-27-4.

No 1155 Beatrice Alenljung: Envisioning a Future Decision Support System for Requirements Engineering - A Holistic and Human-centred Perspective, 2008, ISBN 978-91-85895-11-3. 
No 1156 Artur Wilk: Types for XML with Application to Xcerpt, 2008, ISBN 978-91-85895-08-3.

No 1183 Adrian Pop: Integrated Model-Driven Development Environments for Equation-Based Object-Oriented Languages, 2008, ISBN 978-91-7393-895-2.

No 1185 Jörgen Skågeby: Gifting Technologies Ethnographic Studies of End-users and Social Media Sharing, 2008, ISBN 978-91-7393-892-1.

No 1187 Imad-Eldin Ali Abugessaisa: Analytical tools and information-sharing methods supporting road safety organizations, 2008, ISBN 978-91-7393-887-7.

No 1204 H. Joe Steinhauer: A Representation Scheme for Description and Reconstruction of Object Configurations Based on Qualitative Relations, 2008, ISBN 978-91-7393-823-5.

No 1222 Anders Larsson: Test Optimization for Core-based System-on-Chip, 2008, ISBN 978-91-7393-768-9.

No 1238 Andreas Borg: Processes and Models for Capacity Requirements in Telecommunication Systems, 2009, ISBN 978-91-7393-700-9.

No 1240 Fredrik Heintz: DyKnow: A Stream-Based Knowledge Processing Middleware Framework, 2009, ISBN 978-91-7393-696-5.

No 1241 Birgitta Lindström: Testability of Dynamic RealTime System s, 2009, ISBN 978-91-7393-695-8.

No 1244 Eva Blomqvist: Semi-automatic Ontology Construction based on Patterns, 2009, ISBN 978-91-7393-683-5.

No 1249 Rogier Woltjer: Functional Modeling of Constraint Management in Aviation Safety and Command and Control, 2009, ISBN 978-91-7393-659-0.

No 1260 Gianpaolo Conte: Vision-Based Localization and Guidance for Unmanned Aerial Vehicles, 2009, ISBN 978-91-7393-603-3.

No 1262 AnnMarie Ericsson: Enabling Tool Support for Formal Analysis of ECA Rules, 2009, ISBN 978-91-7393598-2.

No 1266 Jiri Trnka: Exploring Tactical Command and Control: A Role-Playing Simulation Approach, 2009, ISBN 978-91-7393-571-5.

No 1268 Bahlol Rahimi: Supporting Collaborative Work through ICT - How End-users Think of and Adopt Integrated Health Information Systems, 2009, ISBN 978-91-7393-550-0.

No 1274 Fredrik Kuivinen: Algorithms and Hardness Results for Some Valued CSPs, 2009, ISBN 978-91-7393-525-8.

No 1281 Gunnar Mathiason: Virtual Full Replication for Scalable Distributed Real-Time Databases, 2009, ISBN 978-91-7393-503-6.

No 1290 Viacheslav Izosimov: Scheduling and Optimization of Fault-Tolerant Distributed Embedded Systems, 2009, ISBN 978-91-7393-482-4.

No 1294 Johan Thapper: Aspects of a Constraint Optimisation Problem, 2010, ISBN 978-91-7393-464-0.

No 1306 Susanna Nilsson: Augmentation in the Wild: User Centered Development and Evaluation of Augmented Reality Applications, 2010, ISBN 978-917393-416-9.

No 1313 Christer Thörn: On the Quality of Feature Models, 2010, ISBN 978-91-7393-394-0.

No 1321 Zhiyuan He: Temperature Aware and DefectProbability Driven Test Scheduling for System-onChip, 2010, ISBN 978-91-7393-378-0.

No 1333 David Broman: Meta-Languages and Semantics for Equation-Based Modeling and Simulation, 2010, ISBN 978-91-7393-335-3.

No 1337 Alexander Siemers: Contributions to Modelling and Visualisation of Multibody Systems Simulations with
Detailed Contact Analysis, 2010, ISBN 978-91-7393317-9.

No 1354 Mikael Asplund: Disconnected Discoveries: Availability Studies in Partitioned Networks, 2010, ISBN 978-91-7393-278-3.

No 1359 Jana Rambusch: Mind Games Extended: Understanding Gameplay as Situated Activity, 2010, ISBN 978-91-7393-252-3.

No 1373 Sonia Sangari: Head Movement Correlates to Focus Assignment in Swedish, 2011, ISBN 978-91-7393-1540 .

No 1374 Jan-Erik Källhammer: Using False Alarms when Developing Automotive Active Safety Systems, 2011, ISBN 978-91-7393-153-3.

No 1375 Mattias Eriksson: Integrated Code Generation, 2011, ISBN 978-91-7393-147-2.

No 1381 Ola Leifler: Affordances and Constraints of Intelligent Decision Support for Military Command and Control - Three Case Studies of Support Systems, 2011, ISBN 978-91-7393-133-5.

No 1386 Soheil Samii: Quality-Driven Synthesis and Optimization of Embedded Control Systems, 2011, ISBN 978-91-7393-102-1.

No 1419 Erik Kuiper: Geographic Routing in Intermittentlyconnected Mobile Ad Hoc Networks: Algorithms and Performance Models, 2012, ISBN 978-91-7519981-8.

No 1451 Sara Stymne: Text Harmonization Strategies for Phrase-Based Statistical Machine Translation, 2012, ISBN 978-91-7519-887-3.

No 1455 Alberto Montebelli: Modeling the Role of Energy Management in Embodied Cognition, 2012, ISBN 978-91-7519-882-8

No 1465 Mohammad Saifullah: Biologically-Based Interactive Neural Network Models for Visual Attention and Object Recognition, 2012, ISBN 978-91-7519-838-5.

No 1490 Tomas Bengtsson: Testing and Logic Optimization Techniques for Systems on Chip, 2012, ISBN 978-917519-742-5.

No 1481 David Byers: Improving Software Security by Preventing Known Vulnerabilities, 2012, ISBN 97891-7519-784-5.

No 1496 Tommy Färnqvist: Exploiting Structure in CSP related Problems, 2013, ISBN 978-91-7519-711-1.

No 1503 John Wilander: Contributions to Specification, Implementation, and Execution of Secure Software, 2013, ISBN 978-91-7519-681-7.

No 1506 Magnus Ingmarsson: Creating and Enabling the Useful Service Discovery Experience, 2013, ISBN 97891-7519-662-6.

No 1547 Wladimir Schamai: Model-Based Verification of Dynamic System Behavior against Requirements: Method, Language, and Tool, 2013, ISBN 978-917519-505-6.

No 1551 Henrik Svensson: Simulations, 2013, ISBN 978-917519-491-2.

No 1559 Sergiu Rafiliu: Stability of Adaptive Distributed Real-Time Systems with Dynamic Resource Management, 2013, ISBN 978-91-7519-471-4.

No 1581 Usman Dastgeer: Performance-aware Component Composition for GPU-based Systems, 2014, ISBN 978-91-7519-383-0

No 1602 Cai Li: Reinforcement Learning of Locomotion based on Central Pattern Generators, 2014, ISBN 978-917519-313-7.

No 1652 Roland Samlaus: An Integrated Development Environment with Enhanced Domain-Specific 
Interactive Model Validation, 2015, ISBN 978-917519-090-7.

No 1663 Hannes Uppman: On Some Combinatorial Optimization Problems: Algorithms and Complexity, 2015, ISBN 978-91-7519-072-3.

No 1664 Martin Sjölund: Tools and Methods for Analysis, Debugging, and Performance Improvement of Equation-Based Models, 2015, ISBN 978-91-7519-071-6.

No 1666 Kristian Stavåker: Contributions to Simulation of Modelica Models on Data-Parallel Multi-Core Architectures, 2015, ISBN 978-91-7519-068-6.

No 1680 Adrian Lifa: Hardware/ Software Codesign of Embedded Systems with Reconfigurable and Heterogeneous Platforms, 2015, ISBN 978-91-7519-0402.

No 1685 Bogdan Tanasa: Timing Analysis of Distributed Embedded Systems with Stochastic Workload and Reliability Constraints, 2015, ISBN 978-91-7519-022-8.

No 1691 Håkan Warnquist: Troubleshooting Trucks Automated Planning and Diagnosis, 2015, ISBN 97891-7685-993-3.

No 1702 Nima Aghaee: Thermal Issues in Testing of Advanced Systems on Chip, 2015, ISBN 978-91-7685949-0.

No 1715 Maria Vasilevskaya: Security in Embed ded Systems: A Model-Based Approach with Risk Metrics, 2015, ISBN 978-91-7685-917-9.

No 1729 Ke Jiang: Security-Driven Design of Real-Time Embedded System, 2016, ISBN 978-91-7685-884-4.

No 1733 Victor Lagerkvist: Strong Partial Clones and the Complexity of Constraint Satisfaction Problems: Limitations and Applications, 2016, ISBN 978-91-7685856-1.

No 1734 Chandan Roy: An Informed System Development Approach to Tropical Cyclone Track and Intensity Forecasting, 2016, ISBN 978-91-7685-854-7.

No 1746 Amir Aminifar: Analysis, Design, and Optimization of Embedded Control Systems, 2016, ISBN 978-917685-826-4.

No 1747 Ekhiotz Vergara: Energy Modelling and Fairness for Efficient Mobile Communication, 2016, ISBN 978-91-7685-822-6.

No 1748 Dag Sonntag: Chain Graphs - Interpretations, Expressiveness and Learning Algorithms, 2016, ISBN 978-91-7685-818-9.

No 1768 Anna Vapen: Web Authentication using Third Parties in Untrusted Environments, 2016, ISBN 978-91-7685-753-3.

No 1778 Magnus Jandinger: On a Need to Know Basis: A Conceptual and Methodological Framew ork for Modelling and Analysis of Information Demand in an Enterprise Context, 2016, ISBN 978-91-7685-713-7.

No 1798 Rahul Hiran: Collaborative Network Security: Targeting Wide-area Routing and Edgenetw ork Attacks, 2016, ISBN 978-91-7685-662-8.

No 1813 Nicolas Melot: Algorithms and Framework for Energy Efficient Parallel Stream Computing on Many-Core Architectures, 2016, ISBN 978-91-7685623-9.

No 1823 Amy Rankin: Making Sense of Adaptations: Resilience in High-Risk Work, 2017, ISBN 978-917685-596-6.
No 1831 Lisa Malmberg: Building Design Capability in the Public Sector: Expanding the Horizons of Development, 2017, ISBN 978-91-7685-585-0.

No 1851 Marcus Bendtsen: Gated Bayesian Networks, 2017, ISBN 978-91-7685-525-6.

No 1852 Zlatan Dragisic: Completion of Ontologies and Ontology Networks, 2017, ISBN 978-91-7685-522-5.

No 1854 Meysam Aghighi: Computational Complexity of some Optimization Problems in Planning, 2017, ISBN 978-91-7685-519-5.

No 1863 Simon Ståhlberg: Methods for Detecting Unsolvable Planning Instances using Variable Projection, 2017, ISBN 978-91-7685-498-3.

No 1879 Karl Hammar: Content Ontology Design Patterns: Qualities, Methods, and Tools, 2017, ISBN 978-91-7685-454-9.

\section{Linköping Studies in Arts and Science}

No 504 Ing-Marie Jonsson: Social and Emotional Characteristics of Speech-based In-Vehicle Information Systems: Impact on Attitude and Driving Behaviour, 2009, ISBN 978-91-7393-478-7.

No 586 Fabian Segelström: Stakeholder Engagement for Service Design: How service designers identify and communicate insights, 2013, ISBN 978-91-7519-554-4.

No 618 Johan Blomkvist: Representing Future Situations of Service: Prototyping in Service Design, 2014, ISBN 978-91-7519-343-4.

No 620 Marcus Mast: Human-Robot Interaction for SemiAutonomous Assistive Robots, 2014, ISBN 978-917519-319-9.

No 677 Peter Berggren: Assessing Shared Strategic Understanding, 2016, ISBN 978-91-7685-786-1.

No 695 Mattias Forsblad: Distributed cognition in home environments: The prospective memory and cognitive practices of older adults, 2016, ISBN 97891-7685-686-4.

\section{Linköping Studies in Statistics}

No 9 Davood Shahsavani: Computer Experiments Designed to Explore and Approximate Complex Deterministic Models, 2008, ISBN 978-91-7393-976-8.

No 10 Karl Wahlin: Roadmap for Trend Detection and Assessment of Data Quality, 2008, ISBN 978-91-7393792-4.

No 11 Oleg Sysoev: Monotonic regression for large multivariate datasets, 2010, ISBN 978-91-7393-412-1.

No 13 Agné Burauskaite-Harju: Characterizing Temporal Change and Inter-Site Correlations in Daily and Subdaily Precipitation Extremes, 2011, ISBN 978-91-7393110-6.

\section{Linköping Studies in Information Science}

No 1 Karin Axelsson: Metodisk systemstrukturering- att skapa samstämmighet mellan informationssystemarkitektur och verksamhet, 1998. ISBN-9172-19-296-8.

No 2 Stefan Cronholm: Metodverktyg och användbarhet en studie av datorstödd metodbaserad systemutveckling, 1998, ISBN-9172-19-299-2.

No 3 Anders Avdic: Användare och utvecklare - om anveckling med kalkylprogram, 1999. ISBN-91-7219606-8.

No 4 Owen Eriksson: Kommunikationskvalitet hos informationssystem och affärsprocesser, 2000, ISBN 917219-811-7. 
No 5 Mikael Lind: Från system till process - kriterier för processbestämning vid verksamhetsanalys, 2001, ISBN 91-7373-067-X.

No 6 Ulf Melin: Koordination och informationssystem i företag och nätverk, 2002, ISBN 91-7373-278-8.

No 7 Pär J. Ågerfalk: Information Systems Actability - Understanding Information Technology as a Tool for Business Action and Communication, 2003, ISBN 917373-628-7.

No 8 Ulf Seigerroth: Att förstå och förändra systemutvecklingsverksamheter - en taxonomi för metautveckling, 2003, ISBN91-7373-736-4.

No 9 Karin Hedström: Spår av datoriseringens värden Effekter av IT i äldreomsorg, 2004, ISBN 91-7373-9634.

No 10 Ewa Braf: Knowledge Demanded for Action Studies on Knowledge Mediation in Organisations, 2004, ISBN 91-85295-47-7.

No 11 Fredrik Karlsson: Method Configuration method and computerized tool support, 2005, ISBN 91-8529748-8.

No 12 Malin Nordström: Styrbar systemförvaltning - Att organisera systemförvaltningsverksamhet med hjälp av effektiva förvaltningsobjekt, 2005, ISBN 91-8529760-7.

No 13 Stefan Holgersson: Yrke: POLIS - Yrkeskunskap, motivation, IT-system och andra förutsättningar för polisarbete, 2005, ISBN 91-85299-43-X.

No 14 Benneth Christiansson, Marie-Therese Christiansson: Mötet mellan process och komponent - mot ett ramverk för en verksamhetsnära kravspecifikation vid anskaffning av komponentbaserade informationssystem, 2006, ISBN 91-85643$22-X$. 